\title{
Mixed Hodge complexes and higher extensions of mixed Hodge modules on algebraic varieties
}

\author{
FLORIAN IVORRA $(*)$
}

Abstract - In [1, 2] A. Beilinson provides a description of the bounded derived category of polarizable mixed Hodge structures in terms of the more flexible triangulated category of polarizable mixed Hodge complexes. In this work we provide a partial generalization of this result to higher dimension.

Mathematics Subject Classification (2010). 14D07, 14F10, 14F40, 14 F42.

KEYwords. Mixed Hodge modules, mixed Hodge complexes.

\section{Introduction}

\section{1 - Some background}

1.1.1 - In P. Deligne's Hodge theory [5, 8.1], mixed Hodge complexes were introduced as an auxiliary tool to construct mixed Hodge structures on cohomology groups of algebraic complex varieties. This tool was refined by A. Beĭlinson in [1,2] where it plays a crucial role in the definition of absolute Hodge cohomology, a Hodge theoretic precursor of motivic cohomology. More precisely, A. Beillinson introduces a triangulated category of (polarizable) mixed Hodge complexes $\mathrm{D}_{\mathscr{H}}^{p}$ and shows that the triangulated functor

\section{$(1.1 .1-1)$}

$$
\mathrm{D}^{\mathrm{b}}\left(\mathrm{MHS}_{\mathrm{Q}}^{p}\right) \rightarrow \mathrm{D}_{\mathscr{H}^{p}}^{\mathrm{b}}
$$

is an equivalence of categories. Then he explains $[1, \S 4]$ that Deligne's construction provides a mixed Hodge complex $\underline{\mathrm{R}} \Gamma(X, \mathrm{Q})$ which lifts the direct image $\mathbb{R} \pi_{*} Q_{X}$ in Betti cohomology, and, using the equivalence (1.1.1 -1), he defines the absolute (polarizable) Hodge cohomology as the bigraded groups:

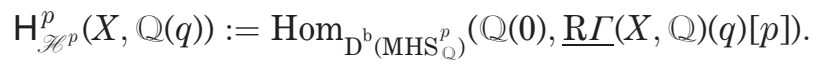

(*) Indirizzo dell'A.: Institut de Recherche Mathématique de Rennes, UMR 6625 du CNRS, Université de Rennes 1, Campus de Beaulieu, 35042 Rennes Cedex(France)

E-mail: florian.ivorra@univ-rennes1.fr 
At the time, $\underline{\mathrm{R}} \Gamma(X, \mathrm{Q})$ was a substitute for the direct image $\pi_{*} \mathrm{Q}_{X}^{\mathscr{H}}$ that a not yet defined theory of Hodge coefficients with a formalism of the six operations would have provided.

1.1.2 - Even with the advent of M. Saito's theory of mixed Hodge modules [17, 19], which allows in particular the definition of absolute Hodge cohomology without mentioning the category of mixed Hodge complexes $\left({ }^{1}\right)$, Bellinson's equivalence still remains a useful description of the bounded derived category of polarizable mixed Hodge structures. Indeed in comparison with complexes of polarizable mixed Hodge structures, mixed Hodge complexes are far more flexible objects and much easier to construct, and the equivalence $(1.1 .1-1)$ turns out to be a key ingredient for constructing realization functors from triangulated categories of motives to the bounded derived category of polarizable mixed Q-Hodge structures $\mathrm{MHS}_{\mathrm{Q}}^{p}$ : in both the construction given by A. Huber in $[7,8]$ for V. Voevodsky's geometrical motives or by M. Levine [14, Part I,V.2.3.10] for his own triangulated category of motives the target category of the realization is rather the bounded triangulated category of polarizable mixed Hodge complexes $\mathrm{D}_{\mathscr{H}}^{\mathrm{b}}$.

1.1.3 - For higher dimensional complex algebraic varieties, categories of mixed Q-Hodge complexes were introduced by M. Saito in [20]. More precisely, for every algebraic variety $X$, he constructs two triangulated categories $\mathrm{D}_{\mathscr{H}}^{\mathrm{b}}(X, Q)_{\mathscr{D}}$ and $\mathrm{D}_{\mathscr{H}}^{\mathrm{b}}(X, Q)$ together with triangulated functors:

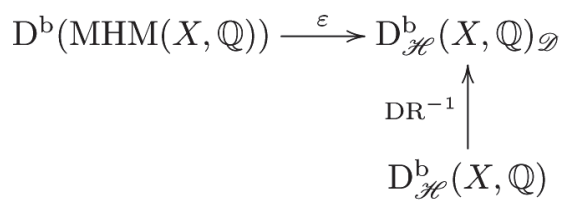

where $\operatorname{MHM}(X, Q)$ is the abelian category of algebraic mixed Q-Hodge modules over $X$. For $X=\operatorname{Spec}(C)$ the categories $\operatorname{MHM}(\operatorname{Spec} C, Q)$ and $\mathrm{MHS}_{Q}^{p}$ are equivalent, M. Saito shows $[20,2.10]$ that the functor $\varepsilon$ in that case

$$
\varepsilon: \mathrm{D}^{\mathrm{b}}(\operatorname{MHM}(\operatorname{Spec} \mathrm{C}, \mathrm{Q})) \rightarrow \mathrm{D}_{\mathscr{H}}^{\mathrm{b}}(\operatorname{Spec} \mathrm{C}, \mathrm{Q})_{\mathscr{D}}
$$

$\left.{ }^{1}\right)$ M. Saito's theory provides a definition of $\pi_{*} \mathrm{Q}_{X}^{\mathscr{G}}$ as an object in $\mathrm{D}^{\mathrm{b}}\left(\mathrm{MHS}_{\mathrm{Q}}^{p}\right)$ and one can shows at least for $X$ smooth that its image by the equivalence (1.1.1 -1) is isomorphic to Beilinson's $\underline{\mathrm{R}} \Gamma(X, \mathrm{Q})$. 
is an equivalence and in particular Saito's category of mixed Hodge complexes is equivalent to $\mathrm{D}_{\mathscr{H}}^{\mathrm{b}}$. Though their definitions are very similar, Saito's construction is slightly more complicated than Beilinson's construction and it is not clear a priori that they yield equivalent results.

1.1.4 - The motivation behind these constructions is the comparison between Hodge structures provided by the theory of mixed Hodge modules and Hodge structures provided by the simplicial methods inherited from Deligne's original approach [5]. As simplicial constructions do not work in the framework of mixed Hodge modules, due to the nonexactness of pullback maps for the perverse $t$-structure, mixed Hodge complexes appears to provide a bridge between the theory elaborated by Saito and other constructions with a simplicial flavor.

\section{2 - Statement of the main result}

1.2.1 - In this paper, for technical reasons, we will have to work with the version of the functor $\varepsilon$ constructed in [9]:

$$
\mathrm{D}^{\mathrm{b}}(\operatorname{MHM}(X, Q)) \stackrel{\text { real }_{X}}{\longrightarrow} \mathrm{D}_{\mathscr{H}}^{\mathrm{b}}(X, \mathrm{Q})_{\mathscr{Q}} .
$$

Though the definition of the category of mixed Hodge complexes $\mathrm{D}_{\mathscr{H}}^{\mathrm{b}}(X, Q)_{\mathscr{Q}}$ is too crude for $(1.2 .1-1)$ to be essentially surjective for an arbitrary algebraic variety, if one is interested in a higher dimensional generalization of Beilinson's equivalence, the following question arises:

1.2.2 - QueSTION. Let X be a smooth projective algebraic variety. Is the realization functor(1.2.1 -1) fully faithful? In other words is the morphism

$(1.2 .2-1) \operatorname{Hom}_{\mathrm{D}^{\mathrm{b}}(\mathrm{MHM}(X, \mathrm{Q}))}(\underline{\mathscr{L}}, \underline{\mathscr{N}}) \rightarrow \operatorname{Hom}_{\mathrm{D}_{\mathscr{H}}^{\mathrm{b}}(X, \mathrm{Q}) \mathscr{\mathscr { C }}}\left(\operatorname{real}_{X}(\underline{\mathscr{L}}), \operatorname{real}_{X}(\underline{\mathscr{N}})\right)$ an isomorphism for any given complexes of mixed Hodge modules $\underline{\mathscr{L}}, \underline{\mathbb{N}}$ ?

1.2.3 - The aim of this work is to provide a partial positive answer to this question for every smooth projective variety. Remark that the realization functor $(1.2 .1-1)$ is a Hodge theoretic lifting of the Betti functor real ${ }_{X, \mathrm{Q}}$ constructed in [4, 3.1], i.e. one has a commutative square (up to an invertible 2-isomorphism) 
$(1.2 .3-1)$

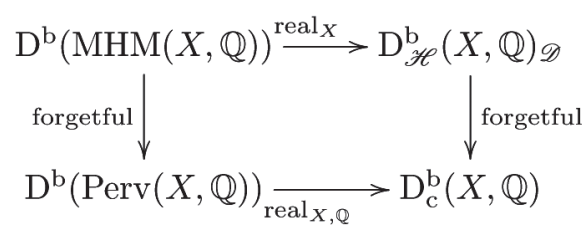

where $\mathrm{D}_{c}^{b}(X, Q)$ is the bounded derived category of sheaves of Q-vector spaces on $X^{\text {an }}$ with algebraically constructible cohomology and $\operatorname{Perv}(X, Q)$ is the abelian category of perverse sheaves inside it. As shown by Beilinson in [3] the functor real ${ }_{X, Q}$ is an equivalence of categories. Therefore a positive answer to 1.2.2 - can be seen both as a higher dimensional generalisation of the equivalence in [1] and a Hodge theoretic version of the equivalence in [3].

1.2.4 - Let us now state our main theorem. For this, recall that if $X$ is a smooth algebraic variety of pure dimension $d_{X}$, an algebraic mixed Q-Hodge module $\mathscr{L}$ on $X$ is said to be smooth if $\operatorname{rat}(\mathscr{C})\left[-d_{X}\right]$ is a local system, where

$$
\text { rat }: \mathrm{D}^{\mathrm{b}}(\mathrm{MHM}(X, \mathrm{Q})) \rightarrow \mathrm{D}_{\mathrm{c}}^{\mathrm{b}}(X, \mathrm{Q})
$$

is the composition of real ${ }_{X, Q}$ and the forgetful functor in $(1.2 .3-1)$. The main result of this work is the following theorem:

1.2.5 - Theorem. Let $X$ be a smooth projective algebraic variety and $\mathscr{l}, \underline{\mathcal{N}}$ be two complexes of mixed Hodge modules on X. If $\mathscr{l l}$ has smooth cohomology i.e. the mixed Hodge module $\mathrm{H}^{i}(\underline{16})$ is smooth for every $i \in \mathrm{Z}$, then the morphism (1.2.2-1) is an isomorphism.

1.2.6 - Let us denote by $\operatorname{MHM}(X, Q){ }_{\mathrm{sm}}$ the full subcategory of $\operatorname{MHM}(X, Q)$ formed by the smooth mixed Q-Hodge modules and by $\mathrm{D}_{\mathrm{sm}}^{\mathrm{b}}(\operatorname{MHM}(X, \mathrm{Q}))$ the full subcategory of $\mathrm{D}^{\mathrm{b}}(\operatorname{MHM}(X, Q))$ formed by the complexes with smooth cohomology. Since $\operatorname{MHM}(X, Q)_{\mathrm{sm}}$ is an abelian subcategory of $\operatorname{MHM}(X, \mathrm{Q})$ stable by extensions, the category $\mathrm{D}_{\mathrm{sm}}^{\mathrm{b}}(\operatorname{MHM}(X, \mathrm{Q}))$ is a triangulated subcategory and 1.2.5 - ensures it can be seen via $(1.2 .1-1)$ as a full triangulated subcategory of $\mathrm{D}_{\mathscr{H}}^{\mathrm{b}}(X, \mathrm{Q})_{\mathscr{D}}$.

1.2.7 - In the proof of 1.2.5 - we use essentially the assumption that $\mathscr{\ell}$ is smooth. Since the definition of $\mathrm{D}_{\mathscr{H}}^{\mathrm{b}}(X, \mathrm{Q})_{\mathscr{O}}$ is really crude in comparison with the definition of the category of mixed Hodge modules, it may be that one has also to refine the construction of mixed Hodge complexes to get a positive answer to 1.2.2 in general. 


\section{3 - Sketch of proof and content of the paper}

1.3.1 - The proof of 1.2.5 - goes by reduction to the zero dimensional case. As shown by M. Saito, a smooth mixed Hodge module is nothing but an admissible variation of mixed Hodge structures. Moreover since such a variation may also be seen as a mixed Hodge complex, to prove 1.2.5 - we have to check that

$$
\operatorname{Hom}_{\mathrm{D}^{\mathrm{b}}(\mathrm{MHM}(X, \mathrm{Q}))}\left(\underline{\mathbb{V}}^{\mathscr{H}}, \underline{\mathscr{N}}\right) \rightarrow \operatorname{Hom}_{\mathrm{D}_{\mathscr{H}}^{\mathrm{b}}(X, \mathrm{Q}) \mathscr{\mathscr { V }}}\left(\underline{\mathbb{V}}^{\mathscr{H}}, \operatorname{real}(\underline{\mathscr{N}})\right)
$$

is an isomorphism for any admissible variation of mixed Hodge structures $\underline{V}$ and any $\mathscr{N} \in \mathrm{D}^{\mathrm{b}}(\mathrm{MHM}(X, \mathrm{Q}))$. The idea of the proof is then very simple:

Step 1 We show that one may assume the variation $\underline{V}$ to be the trivial one $\mathrm{Q}_{X}^{\mathscr{H}}$. To do this reduction, we exploit the fact that the tensor category $\operatorname{VMHS}(\mathrm{X}, \mathrm{Q})_{\mathrm{ad}}$ of admissible variations of mixed Hodge structures is rigid. Namely we construct both on $\mathrm{D}^{\mathrm{b}}(\operatorname{MHM}(X, \mathrm{Q}))$ and $\mathrm{D}_{\mathscr{G}}^{\mathrm{b}}(X, \mathrm{Q})$ a structure of module over $\operatorname{VMHS}(\mathrm{X}, \mathrm{Q})_{\mathrm{ad}}$. The rigidity of the tensor category $\operatorname{VMHS}(\mathrm{X}, \mathrm{Q})_{\text {ad }}$ provides then two isomorphisms

$$
\begin{aligned}
& \operatorname{Hom}_{\mathrm{D}_{\mathscr{H}}^{\mathrm{b}}(X, \mathrm{Q}) \mathscr{\mathscr { T }}}\left(\underline{\mathrm{V}}^{\mathscr{H}}, \underline{\mathscr{C l}}\right)=\operatorname{Hom}_{\mathrm{D}_{\mathscr{H}}^{\mathrm{b}}(X, \mathrm{Q})_{\mathscr{\mathscr { C }}}}\left(\mathrm{Q}_{X}^{\mathscr{H}}, \underline{\mathrm{V}}^{\vee} \circledast \underline{\mathscr{C l}}\right) \\
& \operatorname{Hom}_{\mathrm{D}^{\mathrm{b}}(\operatorname{MHM}(X, \mathrm{Q}))}\left(\underline{\mathbb{V}}^{\mathscr{H}}, \underline{\mathscr{N}}\right)=\operatorname{Hom}_{\mathrm{D}^{\mathrm{b}}(\operatorname{MHM}(X, Q))}\left(Q_{X}^{\mathscr{H}}, \underline{\mathbb{V}}^{\vee} \circledast \underline{\mathscr{N}}\right)
\end{aligned}
$$

where $\mathbb{V}^{v}$ is the dual of $\underline{V}$. We check that the realization functor real is a morphism of modules and the compatibility of the isomorphisms $(1.3 .1-1)$ and (1.3.1 -2) with real ensures that it is enough to consider the trivial variation $Q_{X}^{\mathscr{H}}$. This step does not use the projectivity assumption and relies heavily on the notion of noncharacteristic mixed Hodge modules.

Step 2 Let $\pi: X \rightarrow \operatorname{Spec}(\mathrm{C})$ be the structural morphism. Since $\pi$ is assumed to be projective, we have a direct image functor $\pi_{*}: \mathrm{D}_{\mathscr{G}}^{b}(X, \mathrm{Q})_{\mathscr{Q}} \rightarrow$ $\mathrm{D}_{\mathscr{H}}^{\mathrm{b}}(\operatorname{Spec}(\mathrm{C}), \mathrm{Q}) \mathscr{D}$ for mixed Hodge complexes and we check that there is an isomorphism

$\operatorname{Hom}_{\mathrm{D}_{\mathscr{H}}^{\mathrm{b}}(X, \mathrm{Q})_{\mathscr{\mathscr { C }}}}\left(\mathrm{Q}_{X}^{\mathscr{H}}, \underline{\mathscr{C l}}\right)=\operatorname{Hom}_{\mathrm{D}_{\mathscr{H}}^{\mathrm{b}}(\operatorname{Spec} \mathrm{C}, \mathrm{Q})}\left(\mathrm{Q}(0), \pi_{*} \underline{\mathscr{l l}}\right) \quad \forall \underline{\mathscr{C l}} \in \mathrm{D}_{\mathscr{H}}^{\mathrm{b}}(X, \mathrm{Q})_{\mathscr{Q}}$

which is compatible via real with the adjunction isomorphism in the derived categories of mixed Hodge modules provided by the adjoint functors $\pi^{*} \vdash \pi_{*}$. We are then reduced to the case $\operatorname{Spec}(\mathbb{C})$ which was done in [20, 2.10] or in [1]. 
1.3.2 - Let us now briefly run through the content of the paper. In the second and third sections we provide some complements on $\mathscr{D}$-modules and mixed Hodge modules which are needed as preliminaries to the constructions involved in the proof. Though some of them may be well known to some reader they either do not appear in the literature or the proofs are too quickly sketched for our need. The proof of the main theorem is done in the fifth section while the fourth section contains recollections on mixed Hodge compleces and the last section the necessary though tedious constructions that are needed in the proof but may be skipped in a first reading.

1.3.3 - In this paper a complex algebraic variety is a separated quasiprojective reduced scheme of finite type over Spec C. If $X$ is a smooth complex algebraic variety, we denote by $X^{\text {an }}$ the associated complex analytic manifold. Given an increasing filtration $W$ and an integer $n \in Z$, one denotes by $W\{n\}$ the filtration $W\{n\}_{k}=W_{k-n}$.

\section{Mixed Hodge modules}

\section{1 - Side changing functors for filtered $\mathscr{D}$-modules}

2.1.1 - Let $X$ be a smooth complex algebraic variety or a complex analytic manifold of pure dimension $d_{X}$. We denote by $\mathscr{D}_{X}$ the ring of differential operators on $X$ and by $F$ its filtration by the order. Recall that $\mathscr{D}_{X}=\cup_{k} F_{k} \mathscr{D}_{X}, F_{0} \mathscr{D}_{X}=\mathscr{O}_{X}$ and $F_{k} \mathscr{D}_{X}=0$ for any $k<0$. Moreover $\mathscr{D}_{X}$ is a locally free $\mathscr{O}_{X}$-module and $F_{k} \mathscr{D}_{X}$ is a locally free $\mathscr{O}_{X}$-module of finite rank for any $k \in Z$. A filtered right $\mathscr{D}_{X}$-module is a pair $(\mathscr{L}, F)$ where $\mathscr{L} b$ is a right $\mathscr{D}_{X}$-module and $F_{k} \mathscr{L}(k \in Z)$ is an increasing sequence of additive subgroups of $\mathscr{L} 6$ such that $F_{\ell} \mathscr{L} b \cdot F_{k} \mathscr{D}_{X} \subset F_{k+\ell} \mathscr{l}$. We say that

- $F$ is exhaustive if $\cup_{k} F_{k} \mathscr{L l}=\mathscr{L} b$;

- $F$ is discrete if there exists locally an integer $k_{0} \in Z$ such that $F_{k} \mathscr{L l}=0$ for $k<k_{0}$.

One defines similarly the notion of filtered left $\mathscr{D}_{X}$-modules. In the sequel all filtrations on $\mathscr{D}_{X}$-modules will be assumed to be exhaustive and discrete. The filtered right $\mathscr{D}_{X}$-modules form a quasi-abelian category $\operatorname{MF}\left(\mathscr{D}_{X}\right)$ and we have a forgetful functor $\omega: \operatorname{MF}\left(\mathscr{D}_{X}\right) \rightarrow \mathrm{M}\left(\mathscr{D}_{X}\right)$.

2.1.2 - REMARK. In [17] M. Saito uses right $\mathscr{D}$-modules almost exclusively, here it will be convenient to use also left $\mathscr{D}$-modules. Such a use will 
be indicated by the symbol L e.g. $\mathrm{MF}\left(\mathscr{D}_{X}\right)^{\mathrm{L}}$ will denote the category of filtered left $\mathscr{D}_{X}$-modules.

2.1.3 - Let $\omega_{X}:=\Omega_{X}^{d_{X}}$ be the invertible $\odot_{X}$-module of top differential forms on $X$. This is a right $\mathscr{D}_{X}$-module via the Lie derivative which may also be considered as a filtered right $\mathscr{D}_{X}$-module $\left(\omega_{X}, F\right)$ for the filtration $F$ defined by $\mathrm{Gr}_{-k}^{F} \omega_{X}=0$ if $k \neq d_{X}$. The category of filtered left $\mathscr{D}_{X}$-modules and filtered right $\mathscr{D}_{X}$-modules are equivalent via the side changing functor which associates with a filtered left $\mathscr{D}_{X}$-module $(\mathscr{l}, F)$ the filtered right $\mathscr{D} X$-module

$$
(\mathscr{C l}, F)^{r}:=(\mathscr{C}, F) \otimes_{\mathscr{O}_{X}}\left(\omega_{X}, F\right) .
$$

The underlying right $\mathscr{D}_{X}$-module is $\mathscr{C l}^{r}=\mathscr{\mathscr { C }} \otimes_{O_{X}} \omega_{X}$ and the filtration is given by $F_{k} \cdot \mathscr{C l}^{r}=F_{k+d_{X}} \mathscr{C} \otimes_{\mathscr{O}_{X}} \omega_{X}$. Let $\left(\omega_{X}^{\otimes-1}, F\right)$ be the inverse of $\omega_{X}$ with the filtration $F$ defined by $\mathrm{Gr}_{k}^{F} \omega_{X}^{\otimes-1}=0$ if $k \neq d_{X}$, then given a filtered right $\mathscr{D}_{X}$-module $(\mathscr{C}, F)$

$$
(\mathscr{C l}, F)^{\ell}=\left(\omega_{X}^{\otimes-1}, F\right) \otimes \mathscr{O}_{X}(\mathscr{l}, F)
$$

is the associated filtered left $\mathscr{D}_{X}$-module. We have $F_{k} \mathscr{L}^{\ell}=$ $\omega_{X}^{\otimes-1} \otimes \mathscr{O}_{X} F_{k-d_{X}} \mathscr{l l}$.

2.1.4 - We now add the weight filtration to the picture. Let $\operatorname{MF}\left(\mathscr{D}_{X} ; W\right)$ be the following category. An object is a triple $(\mathscr{C}, F, W)$ where $(\mathscr{C}, F)$ is a filtered right $\mathscr{D}_{X}$-module and $W$ is a finite increasing filtration of $\mathscr{C}$ by sub- $\mathscr{D}_{X}$-modules. A morphism is simply a morphism of filtered $\mathscr{D}_{X^{-}}$ modules which is compatible with the additional filtration $W$. The side changing filtered $\mathscr{D}_{X}$-module $\left(\omega_{X}, F\right)$ is then considered as an object $\left(\omega_{X}, F, W\right)$ in $\operatorname{MF}\left(\mathscr{D}_{X} ; W\right)$ where $W$ is the trivial filtration defined by $\mathrm{Gr}_{n}^{W} \omega_{X}=0$ if $n \neq d_{X}$. The side changing equivalences 2.1.3 extend immediately to equivalences between the categories of $W$-filtered filtered left $\mathscr{D}_{X}$-modules and $W$-filtered filtered right $\mathscr{D}_{X}$-modules. The extension is given by

$$
(\mathscr{C l}, F, W)^{r}:=(\mathscr{C}, F, W) \otimes_{\mathscr{O}_{X}}\left(\omega_{X}, F, W\right)
$$

for a $W$-filtered filtered left $\mathscr{D}_{X}$-module $(\mathscr{C}, F, W)$. In other words the underlying filtered right $\mathscr{D}_{X}$-module is $(\mathscr{C}, F)^{r}$ and the filtration $W$ satisfies $W_{k} \mathscr{C l}^{r}=\left(W_{k-d_{X}} \mathscr{C l}\right)^{r}$. Similarly if $(\mathscr{C}, F, W)$ is a $W$-filtered filtered right $\mathscr{D}_{X}$-module :

$$
(\mathscr{C l}, F, W)^{\ell}=\left(\omega_{X}^{\otimes-1}, F, W\right) \otimes_{\mathscr{C}_{X}}(\mathscr{C l}, F, W)
$$


is the associated $W$-filtered filtered left $\mathscr{D}$-module. In $(2.1 .4-1)$ the filtration $W$ on $\omega_{X}^{\otimes-1}$ is defined by $\mathrm{Gr}_{-n}^{W} \omega_{X}^{\otimes-1}=0$ if $n \neq d_{X}$, the behaviour of the weight filtration is thus given by $W_{k} \mathscr{C} b^{\ell}=\left(W_{k+d_{X}} \mathscr{C l}\right)^{\ell}$.

2.1.5 - Let $\mathscr{C} 6$ be a left $\mathscr{D}_{X}$-module and $\mathrm{DR}(\mathscr{C})$ be its de Rham complex. If $(\mathscr{C}, F)$ is a filtered left $\mathscr{D}_{X}$-module, then $\mathrm{DR}(\mathscr{C})$ inherits a filtration defined for $n \geqslant 0$ and $k \in Z$ by

$$
F_{k} \operatorname{DR}(\mathscr{C l})^{n}=\Omega_{X}^{n} \otimes_{\mathcal{O}_{X}} F_{k+n} \mathscr{C l}
$$

and the resulting filtered complex $\mathrm{DR}(\mathscr{C}, F)$ is a complex of filtered differential operators of order $\leqslant 1$. One sets ${ }^{\mathrm{P}} \mathrm{DR}(\mathscr{C l}):=\mathrm{DR}(\mathscr{C})\left[d_{X}\right]$. Recall that the shift functor does not affect the Hodge filtration i.e $F_{k}{ }^{\mathrm{p}} \mathrm{DR}(\mathscr{C O})^{n}=F_{k} \mathrm{DR}(\mathscr{C O})^{n+d_{X}}$.

2.1.6 - Let $\mathscr{C} 6$ be a right $\mathscr{D}_{X}$-module and $\operatorname{Sp}(\mathscr{C})$ be its Spencer complex. If $(\mathscr{C}, F)$ is a filtered right $\mathscr{D}_{X}$-module, then $\operatorname{Sp}(\mathscr{C})$ inherits a filtration defined for $n \leqslant 0$ and $k \in Z$ by

$$
F_{k} \operatorname{Sp}(\mathscr{C l})^{n}=F_{k+n} \mathscr{C l} \otimes_{O_{X}} \bigwedge^{-n} \Theta_{X}
$$

and the resulting filtered complex $\operatorname{Sp}(\mathscr{C}, F)$ is a complex of filtered differential operators of order $\leqslant 1$.

2.1.7 - If $\mathscr{C}$ is a left $\mathscr{D}$-module then there is a functorial isomorphism

$$
\psi_{\mathscr{C}}: \operatorname{Sp}\left(\mathscr{C l}^{r}\right) \stackrel{\sim}{\rightarrow} \mathrm{p} \mathrm{DR}(\mathscr{C O}) ;
$$

if $(\mathscr{C}, F)$ is a filtered left $\mathscr{D}$-module then this morphism is compatible with the filtrations defined in $(2.1 .5-1),(2.1 .6-1)$ and is therefore an isomorphism of complexes of filtered differential operators of order $\leqslant 1$.

2.1.8 - Let $(\mathscr{C l}, F, W)$ be an object in $\operatorname{MF}\left(\mathscr{D}_{X} ; W\right)$. We denote by $\operatorname{Sp}(\mathscr{C}, W)$ the Spencer complex of $\mathscr{C l}$ endowed with the increasing filtration induced by $W$, we have then

$$
\operatorname{Gr}_{n}^{W} \operatorname{Sp}(\mathscr{C l}, W)=\operatorname{Sp}\left(\mathrm{Gr}_{n}^{W} \mathscr{C}\right) .
$$

Assume that $X$ is a smooth algebraic variety and $(\mathscr{C}, F) \in \operatorname{MF}\left(\mathscr{D}_{X}\right)$, $(\mathscr{N}, F, W) \in \operatorname{MF}\left(\mathscr{D}_{X} ; W\right)$, then one defines

$$
\operatorname{Sp}_{\mathrm{an}}(\mathscr{C l}, F)=\operatorname{Sp}\left(\mathscr{C l}^{\mathrm{an}}\right) \quad \operatorname{Sp}_{\mathrm{an}}(\mathscr{N}, F, W)=\operatorname{Sp}\left((\mathscr{N}, W)^{\mathrm{an}}\right) .
$$

which are (filtered) complexes of sheaves of C-vector spaces on $X^{\text {an }}$. 


\section{2 - Mixed Q-Hodge modules}

2.2.1 - Let $X$ be a smooth algebraic variety of pure dimension $d_{X}$ and $R=\mathrm{Q}$, C. We denote by $\mathrm{D}^{\mathrm{b}}(X, R)$ the bounded derived category of the abelian category of sheaves of $R$-vector spaces on $X^{\text {an }}$. Inside $\mathrm{D}^{\mathrm{b}}(X, R)$ one may consider the strictly full triangulated subcategory $\mathrm{D}_{\mathrm{c}}^{\mathrm{b}}(X, R)$ formed by the complexes $K$ such that $\mathrm{H}^{i}(K)$ is algebraically constructible for every $i \in Z$. Let us denote by $\operatorname{Perv}(X, R)$ the heart of the perverse $t$-structure on $\mathrm{D}_{\mathrm{c}}^{\mathrm{b}}(X, R)$ and by ${ }^{\mathrm{p}} \mathrm{H}^{i}$ the perverse cohomology functors. By the RiemannHilbert correspondence, the de Rham and Spencer functors

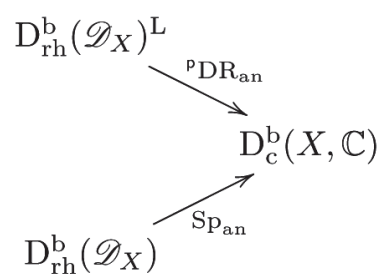

are triangulated equivalences which are exact for the standard $t$-structure on the left hand-sides and the perverse $t$-structure on the right hand-side. In particular, it induces an exact equivalence of abelian categories between $\operatorname{Mod}_{\mathrm{rh}}\left(\mathscr{D}_{X}\right)$ and $\operatorname{Perv}(X, \mathrm{C})$.

2.2.2 - REMARK. For convenience we will consider the category $\operatorname{Perv}(X, R)^{\mathrm{L}}$ obtained by a shift in the definition of perversity: namely $K$ lies in $\operatorname{Perv}(X, R)^{\mathrm{L}}$ if and only if $K\left[d_{X}\right]$ belongs to $\operatorname{Perv}(X, R)$. This is for example the definition of perversity used in [16, 3.3-3]. With this convention a $R$-local system $V$ on $X$ lies in $\operatorname{Perv}(X, R)^{\mathrm{L}}$, and if $\mathscr{C}$ is in $\mathrm{D}_{\mathrm{rh}}^{\mathrm{b}}\left(\mathscr{D}_{X}\right)^{\mathrm{L}}$ then its de Rham and holomorphic solution complexes

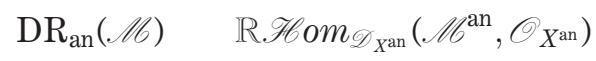

lie also in $\operatorname{Perv}(X, \mathrm{C})^{\mathrm{L}}$.

2.2.3 - Let us recall the definition of the basic category at the bottom of the notion of algebraic Q-Hodge modules introduced by M. Saito (see $[17,5.1 .1])$. Let $\mathrm{MF}_{\mathrm{rh}}\left(\mathscr{D}_{X}\right)$ be the category of filtered right $\mathscr{D}_{X}$-module $(\mathscr{C}, F)$ which satisfy the following two conditions:

- $(\mathscr{l}, F)$ is a coherent filtered $\mathscr{D}_{X}$-module, in other words $F$ is a good filtration on $\mathscr{L l}$;

- $\mathscr{C} 6$ is a regular holonomic $\mathscr{D}_{X}$-module.

Since for an holonomic right $\mathscr{D} X$-module $\mathscr{L}$, the Spencer complex $\operatorname{Sp}_{a n}(\mathscr{C})$ 
is a perverse sheaf, we have a functor

$$
\mathrm{MF}_{\mathrm{rh}}\left(\mathscr{D}_{X}\right) \stackrel{\omega}{\rightarrow} \operatorname{Mod}_{\mathrm{rh}}\left(\mathscr{D}_{X}\right) \stackrel{\mathrm{Sp}_{\mathrm{an}}}{\longrightarrow} \operatorname{Perv}(X, \mathrm{C})
$$

and the following definition is meaningful:

2.2.4 - Definition. The category $\mathrm{MF}_{\mathrm{rh}}\left(\mathscr{D}_{X}, Q\right)$ is the fibre 2-product of the categories $\mathrm{MF}_{\mathrm{rh}}\left(\mathscr{D}_{X}\right)$ and $\operatorname{Perv}(X, \mathrm{Q})$ over $\operatorname{Perv}(X, \mathrm{C})$ :

$$
\operatorname{MF}_{\text {rh }}\left(\mathscr{D}_{X}, Q\right):=\operatorname{MF}_{\text {rh }}\left(\mathscr{D}_{X}\right) \times_{\operatorname{Perv}(X, \mathrm{C})} \operatorname{Perv}(X, Q) .
$$

An object in $\mathrm{MF}_{\mathrm{rh}}\left(\mathscr{D}_{X}\right)$ is called a regular holonomic filtered $\mathscr{D}_{X}$-module with a $Q$-structure.

Let $n \in Z$ be an integer. Via induction on dimension of the support, the category $\mathrm{MH}(X, \mathrm{Q}, n)$ of polarizable Hodge modules of weight $n$ is defined as a strictly full subcategory of $\operatorname{MF}_{\mathrm{rh}}\left(\mathscr{D}_{X}, Q\right)$. The precise definition of a Hodge module of weight $n$ is given in $[17,5.1 .6]$ while the polarizability condition is defined in [17, 5.2.10].

2.2.5 - Remark. Note that we do require pure Hodge modules to be polarizable, this is essential in many respects, the stability of mixed Hodge complexes by proper direct images being only one example where this condition is needed.

By definition an object $\mathscr{C l}$ in $\operatorname{MF}_{\mathrm{rh}}\left(\mathscr{D}_{X}, Q\right)$ is a 5-uplet $\left(K_{\mathrm{Q}}, K_{\mathrm{C}}, K_{\mathscr{Q}}, \alpha \frac{\mathscr{l}}{\mathrm{Q}}, \alpha \frac{\mathscr{H}}{\mathscr{D}}\right)$ where $K_{\mathscr{D}}:=(\mathscr{C}, F)$ is an object in $\mathrm{MF}_{\mathrm{rh}}\left(\mathscr{D}_{X}\right)$, for $R=\mathrm{Q}, \mathrm{C}, K_{R}$ is an object in $\operatorname{Perv}(X, R)$ and $\alpha \frac{\mathscr{L}}{\mathrm{Q}}, \alpha \frac{16}{\mathscr{D}}$ are isomorphisms

$$
K_{\mathrm{Q}} \otimes_{\mathrm{Q}} \mathrm{C} \stackrel{\alpha \frac{\mathscr{Q}}{\mathrm{Q}}}{\longrightarrow} K_{\mathrm{C}} \quad \operatorname{Sp}_{\mathrm{an}}(\mathscr{\mathscr { C }}) \stackrel{\alpha \frac{\mathscr{L}}{\mathscr{Q}}}{\longrightarrow} K_{\mathrm{C}} .
$$

A morphism $\underline{u}: \underline{\mathscr{C}} \rightarrow \mathscr{\mathscr { C }}^{\prime}$ is a triple $\left(u_{\mathrm{Q}}, u_{\mathrm{C}}, u_{\mathscr{O}}\right)$ of morphisms that commute with the comparison morphisms.

2.2.6 - REMARK. The category $\operatorname{MF}_{\mathrm{rh}}\left(\mathscr{D}_{X}, Q\right)$ is also equivalent to the category with objects triples $\underline{\mathscr{C l}}=\left(K_{\mathrm{Q}}, K_{\mathscr{D}}, \alpha_{\mathscr{C l}}\right)$ where $K_{\mathscr{D}}:=(\mathscr{l}, F)$ belongs to $\mathrm{MF}_{\mathrm{rh}}\left(\mathscr{D}_{X}\right), K_{\mathrm{Q}}$ is an object in $\operatorname{Perv}(X, Q)$ and $\alpha_{\mathscr{1 6}}$ : $K_{\mathrm{Q}} \otimes_{\mathrm{Q}} \mathrm{C} \rightarrow \operatorname{Sp}_{\text {an }}(\mathscr{C l})$ is an isomorphism in $\operatorname{Perv}(X, \mathrm{C})$. The morphisms are simply pairs $\left(u_{\mathrm{Q}}, u_{\mathscr{O}}\right)$ of morphisms such that

$$
\alpha_{\underline{\mathscr{C}^{\prime}}} \circ\left(u_{\mathrm{Q}} \otimes_{\mathrm{Q}} \mathrm{C}\right)=\operatorname{Sp}_{\mathrm{an}}\left(u_{\mathscr{D}}\right) \circ \alpha_{\underline{\mathscr{C}}} .
$$

2.2.7 - As in [17, 5.1.14] one defines $\mathrm{MF}_{\mathrm{rh}} \mathrm{W}\left(\mathscr{D}_{X}, Q\right)$ to be the category of objects in $\mathrm{MF}_{\mathrm{rh}}\left(\mathscr{D}_{X}, Q\right)$ endowed with a finite increasing filtration i.e.

$$
\mathrm{MF}_{\mathrm{rh}} \mathrm{W}\left(\mathscr{D}_{X}, \mathbb{Q}\right):=\mathrm{MF}_{\mathrm{rh}}\left(\mathscr{D}_{X}, \mathbb{Q} ; W\right) \times_{\operatorname{Perv}(X, \mathrm{C} ; W)} \operatorname{Perv}(X, \mathrm{Q} ; W)
$$


where $\operatorname{Perv}(X, R ; W)$ is the category of $R$-perverse sheaves with a finite increasing filtration. By definition the abelian category $\operatorname{MHW}(X, Q)$ is the strictly full subcategory of $\mathrm{MF}_{\mathrm{rh}} \mathrm{W}(\mathscr{D}, Q)$ formed by the objects $\mathscr{\mathscr { C }}$ such that

$$
\mathrm{Gr}_{n}^{W} \underline{\mathscr{L l}} \in \mathrm{MH}(X, \mathbb{Q}, n) ;
$$

and by its inductive construction the abelian category of algebraic mixed Hodge modules $\operatorname{MHM}(X, Q)$ is a strictly full subcategory of $\operatorname{MHW}(X, Q)$. Note that the mixed Hodge modules are required to be polarizable.

2.2.8 - When using left $\mathscr{D}_{X}$-modules instead of right $\mathscr{D}_{X}$-modules, it will be convenient to consider the category

$$
\operatorname{MF}_{\text {rh }}\left(\mathscr{D}_{X}, Q\right)^{\mathrm{L}}:=\operatorname{MF}_{\text {rh }}\left(\mathscr{D}_{X}\right)^{\mathrm{L}} \times_{\operatorname{Perv}(X, \mathrm{C})^{\mathrm{L}}} \operatorname{Perv}(X, Q)^{\mathrm{L}}
$$

where the de Rham complex $\mathrm{DR}_{\text {an }}$ of a left $\mathscr{D}_{X}$-module is used instead of the Spencer complex. One defines similarly the category $\mathrm{MF}_{\mathrm{rh}} \mathrm{W}\left(\mathscr{D}_{X}, \mathrm{Q}\right)^{\mathrm{L}}$. The side changing functors 2.1.3-, 2.1.4 - and the isomorphisms 2.1.7provide equivalences

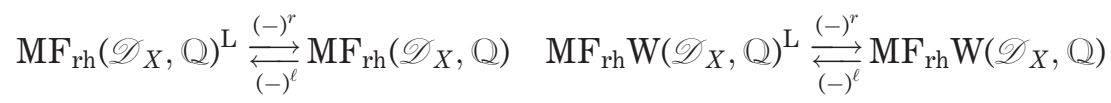

such that for $\underline{\mathscr{C}} \in \mathrm{MF}_{\mathrm{rh}} \mathrm{W}\left(\mathscr{D}_{X}, \mathbb{Q}\right)^{\mathrm{L}}$

$$
\operatorname{Gr}_{n}^{W}\left(\underline{\mathscr{L b}^{r}}\right)=\left(\mathrm{Gr}_{n-d_{X}}^{W} \underline{\mathscr{C l}}\right)^{r}
$$

We therefore define $\mathrm{MH}(X, \mathrm{Q}, n)^{\mathrm{L}}$ to be the subcategory of $\mathrm{MF}_{\mathrm{rh}}\left(\mathscr{D}_{X}, \mathrm{Q}\right)^{\mathrm{L}}$ formed by the objects $\mathscr{\mathscr { C }}$ such that $\mathscr{L}^{r}$ lies in $\mathrm{MH}\left(X, \mathrm{Q}, n+d_{X}\right)$ and the category of left mixed Hodge modules $\operatorname{MHM}(X, Q)^{\mathrm{L}}$ as the subcategory of

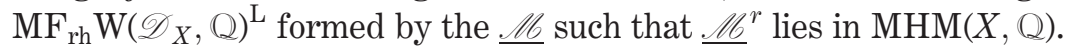

\section{3 - Variations and mixed Hodge modules}

2.3.1 - Let $X$ be a smooth algebraic variety and $\underline{V}$ a variation of mixed Hodge structures on $X$. As part of the definition, we have the following data:

- a pair $\left(\mathbb{V}_{\mathrm{Q}}, W_{\mathrm{Q}}\right)$ where $V_{\mathrm{Q}}$ is a $\mathrm{Q}$-local system $\left(^{2}\right)$ on $X^{\text {an }}$ and $W_{\mathrm{Q}}$ is a finite increasing filtration of $V_{Q}$ by local subsystems;

${ }^{2}$ ) If $A$ is a field a $A$-local system on a topological space is a locally constant sheaf of finite dimensional $A$-vector spaces. 
- a triple $((\mathscr{V}, \nabla), \mathscr{F}, W)$ where $(\mathscr{V}, \nabla)$ is a flat connexion on $X$ with regular singularities at infinity, $\mathscr{F}$ is a finite decreasing filtration of $\mathscr{V}$ by locally free $\mathscr{O}_{X}$-module of finite rank which satisfies Griffiths' tranversality:

$$
\nabla: \mathscr{F}^{k} \mathscr{T} \rightarrow \Omega_{X}^{1} \otimes_{\mathscr{O}_{X}} \mathscr{F}^{k-1} \mathscr{T}
$$

and $W$ is a finite increasing filtration of $\mathscr{V}$ by locally free $\mathscr{O}_{X}$-modules of finite rank which are stable under $\nabla$ :

$$
\nabla: W_{n} \mathscr{V} \rightarrow \Omega_{X}^{1} \otimes_{\mathscr{O}_{X}} W_{n} \mathscr{V}
$$

- an isomorphism of filtered C-local systems

$$
\left(\mathbb{V}_{\mathrm{Q}}, W_{\mathrm{Q}}\right) \otimes_{\mathrm{Q}} \mathrm{C} \stackrel{\alpha_{\underline{\underline{V}}}}{\longrightarrow}\left(\operatorname{Ker} \nabla^{\mathrm{an}}, W\right)
$$

where the flat sections $\operatorname{Ker} \nabla^{\text {an }}$ of the analytic connexion associated with $\nabla$ are endowed with the filtration induced by $W$.

All variations considered in this work are assumed to be polarizable (i.e. for a mixed variation $\underline{\underline{V}}$, all the pure variations $\mathrm{Gr}_{n}^{W} \underline{\mathrm{V}}$ are polarizable). The condition of admissibility was defined in $[21,10]$, the polarizability being part of it. The connexion $\nabla$ defines on $\mathscr{V}$ a structure of left $\mathscr{D} X$-module and setting $F_{k} \mathscr{V}:=\mathscr{F}^{-k} \mathscr{\mathscr { V }}$, Griffiths' transversality condition ensures that $(\mathscr{V}, F)$ is a filtered left $\mathscr{D} X$-module.

2.3.2 - REMARK. For any variation $\underline{V}$ the $\mathscr{O}_{X}$-modules $\mathrm{Gr}_{\mathscr{T}}^{k} \mathrm{Gr}_{n}^{W} \mathscr{T}$ are locally free of finite rank and since $\mathscr{V}$ is an integrable connexion $\operatorname{Char}(\mathscr{V})=T_{X}^{*} X$.

2.3.3 - As shown by M. Saito (see [19, 3.27] and the remark after), the category $\operatorname{VMHS}(\mathrm{X}, \mathrm{Q})_{\mathrm{ad}}$ of admissible variations of Q-mixed Hodge structures is equivalent to the category of smooth mixed Hodge modules, more precisely the equivalence is given by

$$
\begin{aligned}
\operatorname{VMHS}(\mathrm{X}, \mathrm{Q})_{\mathrm{ad}} & \rightarrow \operatorname{MHM}(X, Q)_{\mathrm{sm}} \\
\underline{\mathrm{V}} & \mapsto \underline{\mathrm{V}}^{\mathscr{H}}
\end{aligned}
$$

where $\underline{\mathbb{V}}^{\mathscr{H}}:=\left({ }^{\mathscr{G}} \underline{\mathrm{V}}\right)^{r}$ is the mixed Hodge module obtained by the side changing functor 2.1.4 from the left mixed Hodge module

$$
\mathscr{H}^{\mathscr{V}}:=\left(\left(\nabla_{\mathrm{Q}}, W_{\mathrm{Q}}\right),(\mathscr{V}, F, W), \alpha_{\mathscr{C}} \underline{\mathrm{Y}}\right)
$$


where $\alpha_{\mathbb{V} \mathscr{H}}$ is the filtered isomorphism in the derived category given by the filtered quasi-isomorphism

$$
\alpha_{\mathscr{H} \underline{\mathrm{Y}}}:\left(\mathbb{V}_{\mathrm{Q}}, W_{\mathrm{Q}}\right) \otimes_{\mathrm{Q}} \mathrm{C} \stackrel{\alpha_{\underline{\mathrm{Y}}}}{\longrightarrow} \operatorname{Ker}\left(\nabla^{\mathrm{an}}, W\right) \hookrightarrow \mathrm{DR}_{\mathrm{an}}(\mathscr{V}, W) .
$$

In particular if $\underline{V}$ is a pure variation of weight $n$ then ${ }^{\mathscr{H}} \underline{V}$ lies in $\mathrm{MH}(X, \mathrm{Q}, n)^{\mathrm{L}}$ or equivalently $\underline{V}^{\mathscr{H}}$ belongs to $\mathrm{MH}\left(X, \mathrm{Q}, n+d_{X}\right)$.

\section{Noncharacteristic mixed Hodge modules}

\section{1 - Filtered $\mathscr{D}$-modules and noncharacteristic inverse image}

3.1.1 - Let $f: X \rightarrow Y$ be either a morphism of smooth complex algebraic varieties or a morphism of complex analytic manifolds. Let us say that a $\mathscr{O}_{Y}$-module $\mathscr{E}$ is $\mathscr{O}_{X}$-tor independent if

$$
\mathscr{T}_{0} r_{i}^{f^{-1} \mathscr{O}_{Y}}\left(f^{-1} \mathscr{E}, \mathscr{O}_{X}\right)=0 \quad \forall i>0 .
$$

3.1.2 - Definition. A coherent filtered right $\mathscr{D}_{Y}$-module $(\mathscr{C}, F)$ is said to be noncharacteristic with respect to $f$ if

- $\mathscr{C}$ is noncharacteristic with respect to $f$;

- for any $k \in Z_{s}$ and $i>0$ the $\mathscr{Q}_{Y}$-module $\mathrm{Gr}_{k}^{F} \mathscr{C l}$ is $\mathscr{Q}_{X}$-tor independent i.e.

$$
\mathscr{T}_{O} r_{i}^{f^{-1} \mathscr{O}_{Y}}\left(f^{-1} \mathrm{Gr}_{k}^{F} \mathscr{C l}, \mathscr{O}_{X}\right)=0 .
$$

3.1.3 - REMARK. This is the condition introduced in [17, 3.5.1]. We may give a similar definition for filtered left $\mathscr{D}_{Y}$-modules. Being non characteristic in the sense of 3.1.2 - is then a property stable under the side changing functors 2.1.3 - i.e. a filtered right $\mathscr{D}_{Y}$-module $(\mathscr{C}, F)$ is noncharacteristic if and only if its associated filtered left $\mathscr{D}_{Y}$-module $(\mathscr{C l}, F)^{\ell}$ is.

3.1.4 - Let $f: X \rightarrow Y$ be either a morphism of smooth complex algebraic varieties or a morphism of complex analytic manifolds. Let $d=d_{X}-d_{Y}$ be the relative dimension. As usual, we denote by $\left(\mathscr{D}_{X \rightarrow Y}, F\right)$ and $\left(\mathscr{D}_{Y \leftarrow X}, F\right)$ the filtered transfer modules (see e.g. $[13,5.1]$ for the filtered versions). If $(\mathscr{C}, F)$ is a filtered left $\mathscr{D}_{Y}$-module, one sets

$$
f_{\mathscr{O}}^{*}(\mathscr{C l}, F):=\left(\mathscr{D}_{X \rightarrow Y}, F\right) \otimes_{f^{-1} \mathscr{D}_{Y}} f^{-1}(\mathscr{C l}, F) .
$$

The underlying $\odot_{X}$-module is $\odot_{X} \otimes_{f^{-1} \mathscr{C}_{Y}} f^{-1} \mathscr{C l}$ and the filtration is simply 
given by

$$
F_{k} f_{\mathscr{O}}^{*} \mathscr{C l}:=\operatorname{Im}\left(\odot_{X} \otimes_{f^{-1} \mathscr{O}_{Y}} f^{-1} F_{k} \mathscr{C l} \rightarrow f_{\mathscr{O}}^{*} \mathscr{C b}\right) .
$$

The definition for filtered right $\mathscr{D}_{Y}$-modules goes by the side changing functors, namely if $(\mathscr{C}, F)$ be filtered right $\mathscr{D}_{Y}$-module, then one sets (see $[17,3.5 .1])$

$$
f_{\mathscr{O}}^{*}(\mathscr{C l}, F):=f^{-1}(\mathscr{C l}, F) \otimes_{f^{-1} \mathscr{D}_{Y}}\left(\mathscr{D}_{Y \leftarrow X}, F\right) .
$$

3.1.5 - If $(\mathscr{C}, F, W)$ is a $W$-filtered filtered left- $\mathscr{D}_{X}$-module, one sets

$$
f_{\mathscr{O}}^{*}(\mathscr{C l}, F, W):=\left(\mathscr{D}_{X \rightarrow Y}, F\right) \otimes_{f^{-1} \mathscr{D}_{Y}} f^{-1}(\mathscr{C l}, F, W) .
$$

The filtration $W$ is simply given by

$$
W_{k}\left(f_{\mathscr{O}}^{*} \mathscr{C l}\right):=\operatorname{Im}\left[f_{\mathscr{O}}^{*}\left(W_{k} \mathscr{C b}\right) \rightarrow f_{\mathscr{O}}^{*} \mathscr{\mathscr { C l }}\right] .
$$

The definition for right $\mathscr{D}_{Y}$-modules goes by the side changing functors $2.1 .4-$.

\section{2 - Behaviour of weights in the noncharacteristic case}

3.2.1 - Let $X$ be a smooth algebraic variety. The characteristic variety Char $(\underline{\mathscr{C}})$ of a mixed Hodge module $\underline{\mathscr{C}}$ on $X$ is the characteristic variety of the underlying right $\mathscr{D}$-module. It is a closed subvariety in $T^{*} X$. Though it is an abuse of notation, we will still denote by Char $(\underline{\mathscr{C}})$ the associated closed subset in $T^{*} X^{\text {an }}$.

3.2.2 - Lemma. Let $\underline{\mathscr{L}}$ a mixed Hodge module on $X$. Then in $T^{*} X^{\text {an }}$ $(3.2 .2-1)$

$$
\operatorname{Char}(\underline{\mathscr{C l}})=\mathrm{SS}(\operatorname{rat}(\underline{\mathscr{C l}}))
$$

where SS denotes the microsupport defined by $M$. Kashiwara and $P$. Schapira [12, 5.1.2].

Proof. Let $\mathscr{l} b$ be the left $\mathscr{D}_{X}$-module associated with the underlying right $\mathscr{D}$-module of $\mathscr{C}$ and $\mathbb{D}(\mathscr{C})$ be its dual. Since $\mathscr{C}$ is holonomic by $[12,11.3 .3]$ and e.g. $[6,2.6 .12]$ :

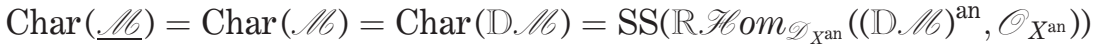

$$
\begin{aligned}
& =\mathrm{SS}\left(\mathbb{R} \cdot \mathscr{H}_{0} m_{\mathscr{D}_{X^{\text {an }}}}\left(\mathrm{D} \cdot \mathscr{l b}^{\mathrm{an}}, \mathscr{O}_{X^{\mathrm{an}}}\right)\right)
\end{aligned}
$$


and the last term is isomorphic to $\operatorname{rat}(\mathscr{C})\left[-d_{X}\right]$ in $\mathrm{D}_{\mathrm{c}}^{\mathrm{b}}(X, \mathrm{Q})$ (see e.g. $[6,4.2 .1])$. Since the microsupport does not change under shift $[12,5.1 .3]$ we have the equality (3.2.2-1) as desired.

3.2.3 - We say that a mixed Hodge module $\mathscr{M b} \in \operatorname{MHM}(Y, Q)$ is noncharacteristic with respect to a morphism $f: X \rightarrow Y$ if and only if for any integer $n \in \mathbb{Z}$ the underlying filtered right $\mathscr{D}_{Y}$-module of $\mathrm{Gr}_{n}^{W} \mathscr{\mathscr { L }}$ is noncharacteristic with respect to $f$ in the sense of 3.1.2.

3.2.4 - Proposition. Let $f: X \rightarrow Y$ be a morphism of smooth algebraic complex varieties and $\mathscr{L} \in \operatorname{MHM}(Y, Q)$. Assume that $\mathscr{L}$ is noncharacteristic with respect to $f$. Then we have an isomorphism

$$
\mathscr{H}^{d} f^{*} \underline{\mathscr{C}} \simeq\left(\mathscr{H}^{-d} f^{!} \underline{\mathscr{C}}\right)(-d)
$$

in $\operatorname{MHM}(X, Q)$.

Proof. Let $\mathscr{L}_{1}$ and $\mathscr{\mathscr { C }}_{2}$ be two objects in $\mathrm{D}^{\mathrm{b}}(\operatorname{MHM}(Y, Q))$. The six operations formalism provides a natural morphism in $\mathrm{D}^{\mathrm{b}}(\mathrm{MHM}(X, \mathrm{Q}))$

$$
f^{!} \underline{\mathscr{L b}_{1}} \otimes f^{*} \underline{\mathscr{L}}_{2} \rightarrow f^{!}\left(\underline{\mathscr{M b}_{1}} \otimes \underline{\mathscr{L}}_{2}\right) .
$$

In particular taking $\mathscr{\mathscr { C }}_{1}=Q_{Y}^{\mathscr{H}}$ and $\mathscr{\mathscr { C }}_{2}=\underline{\mathscr{C}}$ we get a morphism

$$
f^{!} \mathrm{Q}_{Y}^{\mathscr{H}} \otimes f^{*} \underline{\mathscr{L C}} \rightarrow f^{!} \underline{\mathscr{L l}}
$$

which gives the classical morphism (e.g. [12, 3.1.11])

$$
f^{!} \operatorname{rat}\left(Q_{Y}^{\mathscr{H}}\right) \otimes f^{*} \operatorname{rat}(\underline{\mathscr{C l}}) \rightarrow f^{!} \operatorname{rat}(\underline{\mathscr{C l}})
$$

once rat is applied. By 3.2.2 $-\operatorname{rat}(\mathscr{L})$ is noncharacteristic with respect to $f$ in the sense of $[12,5.4 .12]$ and therefore $[12,5.4 .13]$ ensures that $(3.2 .4-2)$ is an isomorphism. Since rat is a conservative functor this implies that (3.2.4-1) is also an isomorphism. Since $Y$ are smooth, we have an isomorphism $\pi_{Y}^{!} \mathrm{Q}^{\mathscr{H}} \simeq \mathrm{Q}_{Y}^{\mathscr{H}}\left(d_{Y}\right)\left[2 d_{Y}\right]$ and similarly for $X$, therefore $f^{!} \mathrm{Q}_{Y}^{\mathscr{H}}$ is isomorphic to $\mathrm{Q}_{X}^{\mathscr{H}}(d)[2 d]$. Hence $(3.2 .4-1)$ provides an isomorphism

$$
f^{*} \underline{\mathscr{L l}}(d)[2 d] \simeq f^{!} \underline{\mathscr{L l}}
$$

and it is enough to apply the cohomological functor $\mathrm{H}^{d}$ to get the proposition.

3.2.5 - Corollary. Under the assumption of 3.2.4-, if $\mathscr{l l}$ is a pure Hodge module of weight $n$ then $\mathscr{H}^{d} f^{*} \mathscr{l l}$ is pure Hodge module of weight $n+d$. 
Proof. Indeed if $\mathscr{L}$ any mixed $\operatorname{Hodge~module~in~} \operatorname{MHM}(Y, Q)$ then we know $[19,2.26]$ that

$\mathrm{Gr}_{i}^{W}\left(\mathscr{H}^{j} f^{*} \underline{\mathscr{C}}\right)=0$ for $i-j>n \quad\left(\operatorname{resp} . \mathrm{Gr}_{i}^{W}\left(\mathscr{H}^{j} f^{!} \underline{\mathscr{C l}}\right)=0\right.$ for $\left.i-j<n\right)$ if $\operatorname{Gr}_{i}^{W} \mathscr{\mathscr { L }}=0$ for $i>n$ (resp. $i<n$ ). Hence the purity assumption implies $\left(^{3}\right)$ on the one hand that

$$
\operatorname{Gr}_{i}^{W}\left(\mathscr{H}^{d} f^{*} \underline{\mathscr{C l}}\right)=0 \text { for } i>n+d
$$

and on the other hand that

$$
\operatorname{Gr}_{i}^{W}\left(\left(\mathscr{H}^{-d} f^{!} \underline{\mathscr{C}}\right)(-d)\right)=0 \text { for } i n+d .
$$

The corollary follows then from 3.2.4 -.

\section{3 - A remark on Cauchy-Kovalevskaya-Kashiwara theorem}

3.3.1 - Let $f: X \rightarrow Y$ be a morphism of complex manifolds. We let $d: d_{X}-d_{Y}$ be the relative dimension. Let $\mathscr{l}, \mathscr{N}$ in $\mathrm{D}_{\text {coh }}^{\mathrm{b}}\left(\mathscr{D}_{Y}\right)$. Consider the canonical morphism in $\mathrm{D}^{\mathrm{b}}(X, \mathrm{C})$

$$
\operatorname{can}(f, \mathscr{L}, \mathscr{N}): f^{-1} \mathbb{R} \mathscr{H}_{0} o m_{\mathscr{D}_{Y}}(\mathscr{L}, \mathscr{N}) \rightarrow \mathbb{R} \mathscr{H} \mathscr{C o m}_{\mathscr{D}_{X}}\left(\mathbb{L}_{\mathscr{O}}^{*} \mathscr{L l}, \mathbb{L}_{\mathscr{O}}^{*} \mathscr{N}\right)
$$

(see e.g. [6, p. 106] for its definition), which provides in particular the following morphisms

$(3.3 .1-1) \operatorname{can}\left(f, \mathscr{l}, \mathscr{O}_{Y}\right): f^{-1} \mathbb{R} \mathscr{H}_{0} m_{\mathscr{D}_{Y}}\left(\mathscr{l}, \mathscr{O}_{Y}\right) \rightarrow \mathbb{R} \mathscr{H} O m_{\mathscr{D}_{X}}\left(\mathrm{~L}_{\mathscr{O}}^{*} \mathscr{L l}, \mathscr{O}_{X}\right)$

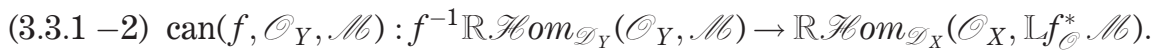

Assume that $\mathscr{C}$ is noncharacteristic with respect to $f$. Then $(3.3 .1-1)$ is an isomorphism. This result is the generalization of the Cauchy-Kovalevskaya theorem proven by M. Kashiwara in [11] (see also e.g. [6, Theorem 4.3.2]). Using duality, it follows that (3.3.1 -2) is also an isomorphism. However this is a morphism in the derived category. In the sequel, see Section 6 , we rather need a genuine morphism of complexes that represents it. We provide now its definition.

3.3.2 - For this let us remark that, given a left $\mathscr{D}_{Y}$-module $\mathscr{L}$, there exists a functorial morphism of complexes of $\mathrm{C}_{X}$-modules

$$
f_{\mathscr{C}}^{b}: f^{-1} \mathrm{DR}(\mathscr{C O}) \rightarrow \mathrm{DR}\left(f_{\mathscr{O}}^{*} \mathscr{C l}\right)
$$

$\left({ }^{3}\right)$ The Tate twist is defined in [19, 2.17.7]. 
which for $\mathscr{l}=\mathscr{O}_{Y}$ coincides with the canonical morphism $f^{-1} \Omega_{Y} \rightarrow \Omega_{X}$. The $n$-th component of (3.3.2-1) is the morphism of sheaves

$$
\left(f_{\mathscr{C}}^{b}\right)^{n}: f^{-1} \Omega_{Y}^{n} \otimes_{f^{-1} \mathscr{O}_{Y}} f^{-1} \mathscr{C l} \rightarrow \Omega_{X}^{n} \otimes_{\mathscr{O}_{X}} f_{\mathscr{O}}^{*} \mathscr{C}
$$

defined for a local section $\omega \in \Omega_{Y}^{n}$ and a local section $m \in \mathscr{C l}$ by

$$
\left(f_{\mathscr{C}}^{b}\right)^{n}(\omega \otimes m)=f^{*} \omega \otimes(1 \otimes m) .
$$

3.3.3 - REMARK. This formula defines a morphism of complexes. Indeed by the Leibniz formula it is enough to consider the case where $\omega$ is a function in $\mathscr{Q}_{Y}$ and we may further assume $\omega=1$. We may further assume that we have a coordinate system $\left(y_{1}, \ldots, y_{s}\right)$ on $Y$ and a coordinate system $\left(x_{1}, \ldots, x_{r}\right)$ on $X$. We have then for a local section $m \in \mathscr{C l}$

$$
\begin{aligned}
f_{\mathscr{C}}^{b}\left(\nabla_{\mathscr{C l}}(1 \otimes m)\right) & =f_{\mathscr{C}}^{b}\left(\sum_{j=1}^{s} d y_{j} \otimes \partial_{y_{j}} m\right)=\sum_{j=1}^{s} f^{*} d y_{j} \otimes\left(1 \otimes \partial_{y_{j}} m\right) \\
& =\sum_{j=1}^{s} \sum_{i=1}^{r} \partial_{x_{i}} f_{j} d x_{i} \otimes\left(1 \otimes \partial_{y_{j}} m\right)=\sum_{i=1}^{r} d x_{i} \otimes\left(\sum_{j=1}^{s} \partial_{x_{i}} f_{j} \otimes \partial_{y_{j}} m\right) \\
& =\sum_{i=1}^{r} d x_{i} \otimes\left(\sum_{j=1}^{s} \partial_{x_{i}} f_{j} \otimes \partial_{y_{j}} m\right)=\sum_{i=1}^{r} d x_{i} \otimes \partial_{x_{i}}(1 \otimes m) \\
& =\nabla_{f_{\mathscr{O}}^{*} \mathscr{C}}\left(f_{\mathscr{L}}^{b}(1 \otimes m)\right) .
\end{aligned}
$$

3.3.4 - Recall that given a left $\mathscr{D}_{X}$-module $\mathscr{C}$, one has a canonical isomorphism of complexes DR $(\mathscr{C})=\mathscr{H}_{0} \mathrm{~m}_{\mathscr{D}_{X}}\left(\operatorname{Sp}\left(\mathscr{D}_{X}\right), \mathscr{C}\right)$. Since $\operatorname{Sp}\left(\mathscr{D}_{X}\right)$ is a resolution of $\mathscr{O}_{X}$ by locally free $\mathscr{D}_{X}$-module, this isomorphism provides a natural isomorphism

$$
\operatorname{co}(\mathscr{C l}): \operatorname{DR}(\mathscr{C l}) \rightarrow \operatorname{R} \mathscr{H} \mathrm{Om}_{\mathscr{D}_{X}}\left(\odot_{X}, \mathscr{C b}\right)
$$

in $\mathrm{D}^{\mathrm{b}}(X, \mathrm{C})$.

3.3.5 - LemMA. Let $\mathscr{l}$ be a coherent left $\mathscr{D}_{Y}$-module. If $\mathscr{l l}$ is noncharacteristic with respect to $f$, then the morphism $(3.3 .2-1)$ represents the morphism $\left(^{4}\right)$

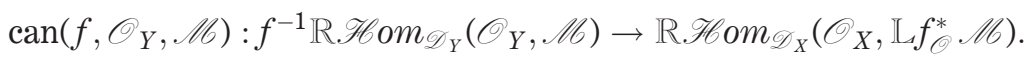

In particular (3.3.2-1) is a quasi-isomorphism.

$\left({ }^{4}\right)$ Recall that if $\mathscr{C}$ is noncharacteristic coherent $\mathscr{D}_{Y}$-module, then $\mathbb{L}_{f} f_{\mathscr{O}}^{*} \mathscr{\mathscr { C }}$ is concentrated in degree 0 and thus $\mathrm{L}_{\mathscr{O}}^{*} \mathscr{\mathscr { C }}=f_{\mathscr{O}}^{*} \mathscr{\mathscr { L }}$. 
3.3.6 - REMARK. The filtered inverse image of a differential complex of order $\leqslant 1$ is given by the tensor product

$$
f_{\text {Diff }}^{*}(\mathscr{l}, F):=\left(\Omega_{X}, F\right) \otimes_{f^{-1} \Omega_{Y}} f^{-1}(\mathscr{C l}, F) .
$$

In particular the filtration on the inverse image is given by

$$
F_{k}\left(f_{\text {Diff }}^{*} \mathscr{C G}\right)^{n}=\sum_{p+q=n} \operatorname{Im}\left(\Omega_{X}^{p} \otimes_{f^{-1} \mathscr{O}_{Y}} f^{-1} F_{k+p} \mathscr{C}^{q}\right)
$$

and the canonical morphism of DG-algebras $f^{-1} \Omega_{Y} \rightarrow \Omega_{X}$ induces therefore a morphism

$$
f_{\mathscr{L} 6}^{b}: f^{-1}(\mathscr{C l}, F) \rightarrow f_{\text {Diff }}^{*} \mathscr{C l} .
$$

Another way to interpret the morphism $(3.3 .2-1)$ is to remark that one has a canonical isomorphism of complexes

$$
f_{\text {Diff }}^{*} \operatorname{DR}(\mathscr{C l}):=\Omega_{X} \otimes_{f^{-1} \Omega_{Y}} f^{-1} \operatorname{DR}(\mathscr{C l}) \stackrel{\sim}{\rightarrow} \mathrm{DR}\left(f_{\mathscr{O}}^{*} \mathscr{C l}\right) .
$$

With this description of the de Rham complex of the inverse image, (3.3.2 -1) is then nothing but the morphism induced by the usual morphism $f^{-1} \Omega_{Y} \rightarrow \Omega_{X}$.

3.3.7 - Remark. The morphism (3.3.2-1) is functorial in $\mathscr{l}$, but it is also compatible with the composition of morphisms. More precisely, if

$$
W \stackrel{g}{\rightarrow} X \stackrel{f}{\rightarrow} Y
$$

are morphisms of smooth complex algebraic varieties, then

$$
(f g)_{\mathscr{C O}}^{b}=g_{f_{O}^{*} \mathscr{C O}}^{b} \circ g^{-1} f_{\mathscr{C O}}^{b}
$$

for any left $\mathscr{D}_{Y}$-module $\mathscr{C}$.

If $\mathscr{C} b$ is a right $\mathscr{D}_{Y}$-module, using the isomorphism given in $2.1 .7-$, we get a morphism of complexes of $\mathrm{C}_{X}$-modules

$$
f_{\mathscr{C l}}^{b}: f^{-1} \operatorname{Sp}_{X}(\mathscr{C l})[d] \rightarrow \operatorname{Sp}\left(f_{\mathscr{O}}^{*} \mathscr{C l}\right)
$$

which is functorial in $\mathscr{C}$. Moreover 3.3.7 - translates into the following equality

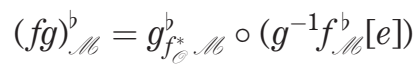

for a sequence of morphisms $W \stackrel{g}{\rightarrow} X \stackrel{f}{\rightarrow} Y$ of smooth algebraic varieties. In (3.3.7-2) the integer $e$ is the relative dimension $e=d_{W}-d_{X}$ of the morphism $g$. 


\section{4 - Noncharacteristic inverse image}

3.4.1 - Let $\underline{\mathscr{C}}=\left((\mathscr{C}, F), K_{\mathrm{Q}}, \alpha_{\mathscr{\ell}}\right)$ be an object in $\operatorname{MF}_{\mathrm{rh}}\left(\mathscr{D}_{Y}, \mathrm{Q}\right)$ and $f: X \rightarrow Y$ a morphism of smooth complex algebraic varieties of relative dimension $d$. Assume that $\underline{\mathscr{C}}$ is noncharacteristic with respect to $f$.

3.4.2 - REMARK. By 3.2.2 - the perverse sheaf $K_{\mathrm{Q}}$ is noncharacteristic with respect to $f$, in the sense of Kashiwara-Schapira. Hence

$$
f^{-1} K_{\mathrm{Q}}[d]=f^{!} K_{\mathrm{Q}}[-d]
$$

is a perverse sheaf on $X^{\text {an }}$. Moreover $f_{\mathscr{O}}^{*}(\mathscr{C l}, F)$ is a regular holonomic filtered right $\mathscr{D}_{X}$-module i.e. belongs to $\operatorname{MF}_{\text {rh }}\left(\mathscr{D}_{X}\right)$.

The morphism (3.3.7-1) defined at the level of the complexes provides an isomorphism $\alpha_{f_{\mathscr{O}}^{*} \mathscr{\ell}}$ in $\operatorname{Perv}(X, \mathrm{C})$ :

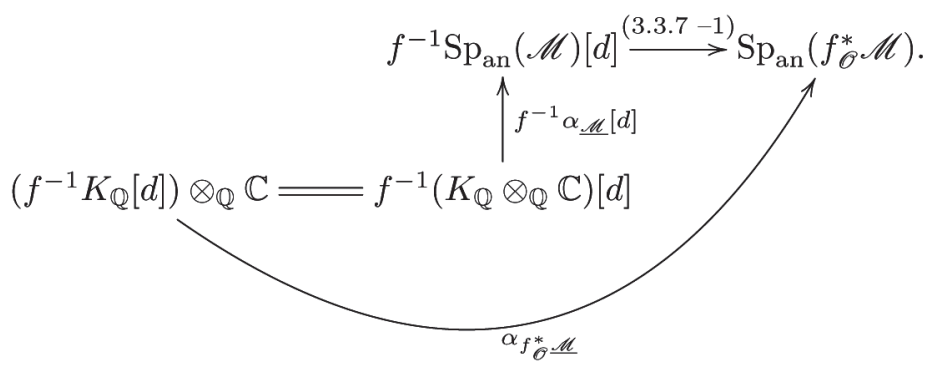

Therefore, we have a well defined object

$$
f_{\mathscr{O}}^{*} \underline{\mathscr{C}}:=\left(f_{\mathscr{O}}^{*}(\mathscr{C}, F), f^{-1} K_{\mathrm{Q}}[d], \alpha_{f_{\mathscr{O}}^{*} \mathscr{\mathscr { C }}}\right)
$$

in $\mathrm{MF}_{\mathrm{rh}}\left(\mathscr{D}_{X}, \mathrm{Q}\right)$. The construction is functorial for noncharacteristic objects in $\mathrm{MF}_{\mathrm{rh}}\left(\mathscr{D}_{Y}, \mathrm{Q}\right)$.

3.4.3 - Proposition. Let $\underline{\mathscr{l}} \in \mathrm{MH}(Y, \mathrm{Q}, n)$ be a Hodge module of weight $n$ and $f: X \rightarrow Y$ a morphism of smooth complex algebraic varieties. Assume that $\underline{\mathscr{L}}$ is noncharacteristic for $f$. Then, its noncharacteristic inverse image $f_{\mathscr{O}}^{*} \underline{\mathscr{L}}$ is a Hodge module on $X$ of weight $n+d$.

3.4.4 - Let us start now the recollections on vanishing cycles which are necessary to write down the proof of 3.4.3-. In order to rely more easily on [15] we will use the convention in loc.cit., in particular we will use left $\mathscr{D}$-modules and left perverse sheaves as in 2.2.2-. Let $X$ be a smooth algebraic complex variety and $H$ be a smooth hypersurface in $X$ defined by a global equation $g \in \Gamma\left(X, \odot_{X}\right)$. Denote by $i: H \hookrightarrow X$ the closed 
immersion. Consider the following diagram

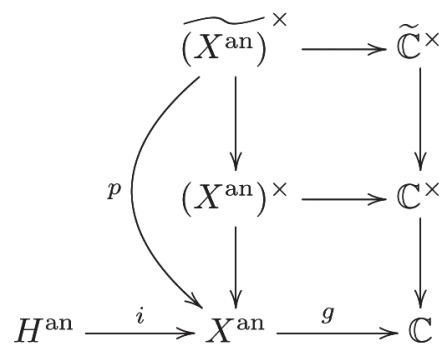

where $\widetilde{\mathrm{C}}^{\times}$denote the universal cover of the punctured complex plane $\mathbb{C}^{\times}:=\mathbb{C} \backslash\{0\}$. Let $R=\mathrm{Q}, \mathbb{C}$ and $K \in \mathrm{D}_{\mathrm{c}}^{\mathrm{b}}(X, R)$, one has a distinguished triangle in $\mathrm{D}_{\mathrm{c}}^{b}(H, R)$

$$
i^{-1} K \rightarrow \Psi_{g}(K) \rightarrow \Phi_{g}(K) \stackrel{+1}{\longrightarrow}
$$

where $\Psi_{g}(K):=i^{-1} \mathbb{R} p_{*} p^{-1} K$ is the nearby cycles complex and $\Phi_{g}(K)$ is the vanishing cycles complex. If $K$ is a left perverse sheaf on $X^{\text {an }}$, then both $\Psi_{g}(K)$ and $\Phi_{g}(K)$ are left perverse sheaves on $H^{\text {an }}$.

3.4.5 - Let $\mathscr{C} 6$ be a left algebraic $\mathscr{D}_{X}$-module. Assume that $\mathscr{C} 6$ is regular holonomic. Then by [15, 4.4-2] $\mathscr{C} 6$ is specializable along $H$ and we denote by $V$ the canonical $V$-filtration of $\mathscr{C} 6$ defined by the relative order with respect to $H$. The nearby and vanishing cycles $\mathscr{D}_{H}$-modules of $\mathscr{C l}$ are defined as

$$
\begin{aligned}
\Psi_{g}(\mathscr{C l}) & :=\left.\bigoplus_{-1 \leqslant \alpha<0} \operatorname{Gr}_{\alpha}^{V}(\mathscr{C l})\right|_{H} ; \\
\Phi_{g}(\mathscr{C l}) & :=\left.\bigoplus_{-1<\alpha \leqslant 0} \operatorname{Gr}_{\alpha}^{V}(\mathscr{C l})\right|_{H} .
\end{aligned}
$$

They are regular holonomic $\mathscr{D}_{H}$-modules $[15,4.7-5]$ and one has an isomorphism (functorial in 16 ) $[15,5.3-2]$ of left perverse sheaves on $H^{\text {an }}$

$$
\operatorname{can}_{g, \mathscr{l}}^{\Psi}: \Psi_{g}\left(\mathrm{DR}_{X^{\text {an }}}\left(\mathscr{C l}^{\text {an }}\right)\right) \rightarrow \mathrm{DR}_{H^{\text {an }}}\left(\Psi_{g}(\mathscr{C l})^{\text {an }}\right)
$$

and a similar isomorphism $\operatorname{can}_{g, \mathscr{C}}^{\Phi}$ for the vanishing cycles. If $(\mathscr{C}, F)$ is a regular holonomic filtered left $\mathscr{D}_{X}$-module which is quasi-unipotent and regular along $H$ in the sense of [17, 3.2.1], then the filtered nearby and vanishing cycles functors are endowed with the filtration induced $\left(^{5}\right)$ by $F$ and belong to $\operatorname{MF}_{\mathrm{rh}}\left(\mathscr{D}_{H}\right)^{\mathrm{L}}$.

$\left.{ }^{5}\right)$ For filtered right $\mathscr{D}_{X}$-module, the vanishing cycles are defined similarly in $[17,5.1 .3 .3]$ but endowed by the filtration induced by $F\{1\}$. The definition for filtered left $\mathscr{D}$-modules considered here is compatible with M. Saito's definition via the side-changing functors $2.1 .3-$. 
3.4.6 - Let $\mathscr{\mathscr { C }} \in \mathrm{MF}_{\mathrm{rh}}\left(\mathscr{D}_{X}, Q\right)^{\mathrm{L}}$ such that the underlying filtered left $\mathscr{D}_{X^{-}}$ module is quasi-unipotent and regular along $H$, then the isomorphism (3.4.5 - 1) provide an object in $\mathrm{MF}_{\mathrm{rh}}\left(\mathscr{D}_{H}, Q\right)^{\mathrm{L}}$

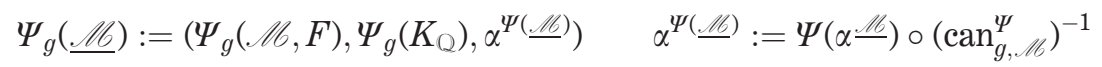

and one defines similarly $\Phi_{g}(\mathscr{\mathscr { L }})$. By $[15,5.3-2]$ we have a commutative diagram in the category Perv $\left(H^{\text {an }}, \mathrm{C}\right)^{\mathrm{L}}$

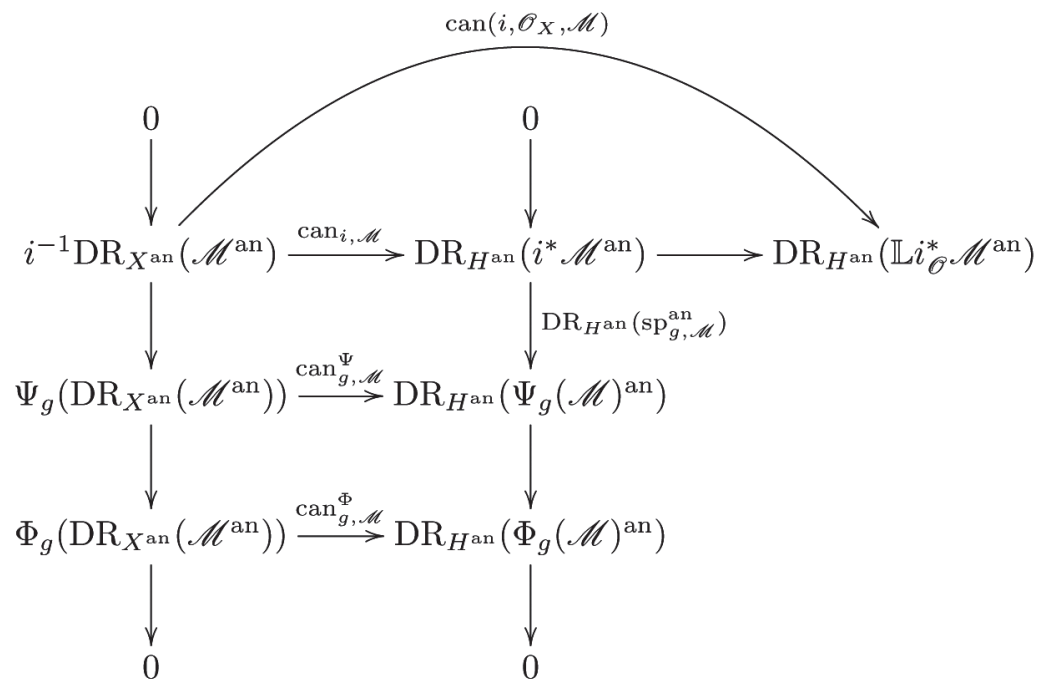

such that the vertical sequences are short exact sequences. The morphisms $i^{*} \mathscr{L} \rightarrow \mathbb{L}^{*} \mathscr{O} \mathscr{C}$ and $\mathrm{sp}_{\mathrm{g}, \mathscr{C}}$ are given (functorially in $\mathscr{C O}$ ) in the derived category by the morphisms of complexes [15, 4.4-4,4.6-4]
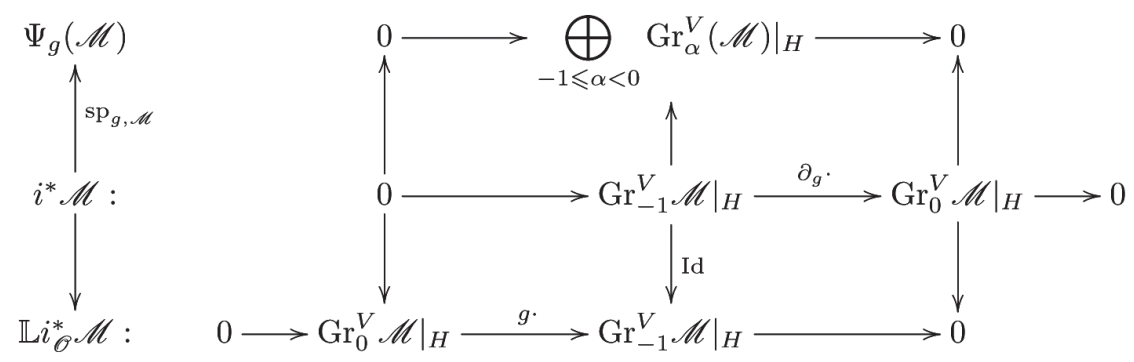

where the middle term $\left.\mathrm{Gr}_{-1}^{V} \mathscr{\mathscr { C }}\right|_{H}$ lies in degree 0 . If $(\mathscr{C}, F)$ is noncharacteristic with respect to $i$, then $(\mathscr{L}, F)$ is quasi-unipotent and regular along $H[17,3.5 .6]$ and we have $\operatorname{Gr}_{\alpha}^{V}(\mathscr{C l}, F)=0$ as soon as $\alpha \notin\{-1,-2, \ldots\}$. 
This implies that $\Phi_{g} \mathscr{C l}=0$ and that $N=0$ on $\Psi_{g}(\mathscr{C l})$. We have therefore an isomorphism $\mathrm{MF}_{\text {rh }}\left(\mathscr{D}_{H}, \mathrm{Q}\right)^{\mathrm{L}}$

$$
i_{\mathscr{O}}^{*} \underline{\mathscr{C l}} \simeq \Psi_{g}(\underline{\mathscr{L C}})
$$

and the nilpotent monodromy operator $N$ on $\Psi_{g}(\mathscr{C})$ vanishes. Using the side changing functors we get a similar result for right $\mathscr{D}$-modules.

3.4.7 - Assume now that $\mathscr{L}$ is a pure Hodge module of weight $n$ on $X$ (we use now the convention in [17] and thus in particular right $\mathscr{D}$-modules). Let

$$
g: X \stackrel{i_{g}}{\longrightarrow} X \times \mathrm{A}^{1} \stackrel{\pi}{\longrightarrow} \mathrm{A}^{1}
$$

be the factorization of $g$ by its graph. By [17, 3.2.4,3.2.5] we have a canonical isomorphism

$$
\psi_{g}(\underline{\mathscr{L l}}) \simeq i_{*} \Psi_{g}(\underline{\mathscr{L l}})
$$

where $\psi_{g}(\mathscr{L C}):=\Psi_{\pi}\left(i_{g *} \mathscr{\mathscr { C }}\right)$ is the nearby cycles functor as defined by M. Saito [17, 5.1.3.3]. Since $N$ is trivial on $\psi_{g}(\mathscr{L C})$, the associated monodromy filtration $\mathrm{M}$ is trivial and therefore the inductive construction of the category of pure Hodge modules [17, 5.1.6.2] implies that

$$
\operatorname{Gr}_{i}^{\mathrm{M}\{n-1\}} \psi_{g}(\underline{\mathscr{C}})=\operatorname{Gr}_{i-n+1}^{\mathrm{MI}} \psi_{g}(\underline{\mathscr{C}})= \begin{cases}\psi_{g}(\underline{\mathscr{C}}) & \text { if } i=n-1 \\ 0 & \text { otherwise }\end{cases}
$$

is a pure Hodge module of weight $i$ on $X$. Then, it follows from (3.4.7-1), $(3.4 .6-1)$ and $[17,5.1 .9]$ that $i_{\mathscr{O}}^{*} \mathscr{L} 6$ is a pure Hodge module of weight $n-1$ on $H$ as desired.

Proof OF 3.4.3-. Let

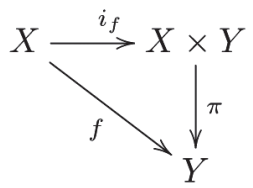

be the factorization of $f$ via its graph. By [17, 3.5.4], it is enough to check the result for $\pi$ and $i_{f}$. For $\pi$ the result is a consequence of $[19,3.21]$ (see also [19, 3.28]) since one has an isomorphism

$$
\pi_{\mathscr{O}}^{*} \underline{\mathscr{M b}}=\mathbb{Q}_{X}^{\mathscr{H}}\left[d_{X}\right] \otimes \underline{\mathscr{C l}}
$$

and $Q_{X}^{\mathscr{H}}\left[d_{X}\right]$ is a pure Hodge module of weight $d_{X}$. Let us now prove the result for any closed immersion $i: X \hookrightarrow Y$ by induction on the codimension 
of $X$ in $Y$. Since locally on $X$ we may factor $i$ as a composition of immersions of codimension one between smooth closed subschemes, by [17, 3.5.4] and the fact that the conditions which define Hodge modules are of local nature $[17,5.1 .7]$, we are reduced to the case of a smooth closed hypersurface which was considered above.

3.4.8 - Let $\mathscr{\mathscr { C }}=\left((\mathscr{C l}, F, W),\left(K_{\mathrm{Q}}, W_{\mathrm{Q}}\right), \alpha_{\mathscr{C}}\right)$ be an object in $\mathrm{MF}_{\mathrm{rh}} \mathrm{W}\left(\mathscr{D}_{Y}, \mathrm{Q}\right)$ and $f: X \rightarrow Y$ a morphism of smooth complex algebraic varieties of relative dimension $d$. Assume that $\mathscr{C}$ is noncharacteristic with respect to $f$. The morphism $(3.3 .7-1)$ defined at the level of the complexes provides an isomorphism $\alpha_{f_{\odot}^{*}, \underline{L}}$ in $\operatorname{Perv}(X, \mathrm{C})$ :

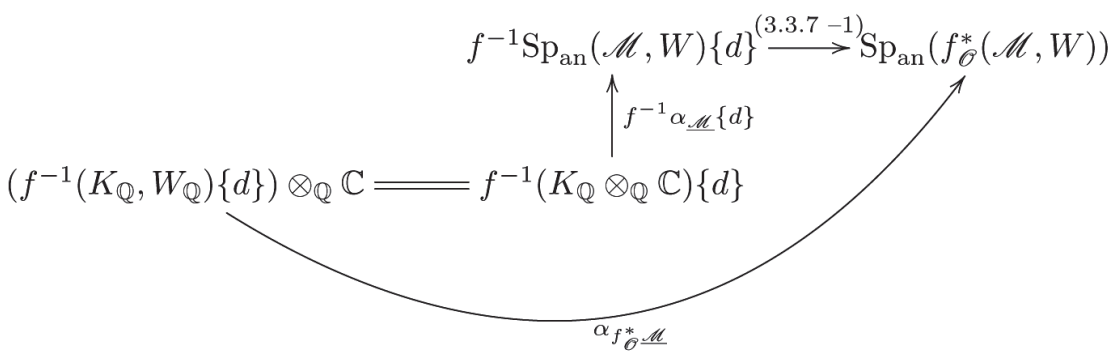

where $(K, W)\{d\}:=(K, W\{d\})[d]$ and $W\{d\}_{k}:=W_{k-d}$. Therefore, we have a well defined object

$$
f_{\mathscr{O}}^{*} \underline{\mathscr{L l}}:=\left(f_{\mathscr{O}}^{*}(\mathscr{\mathscr { C }}, F, W), f^{-1}\left(K_{\mathrm{Q}}, W_{\mathrm{Q}}\right)\{d\}, \alpha_{f_{\mathscr{O}}^{*}} \underline{\mathscr{\ell}}\right)
$$

in $\mathrm{MF}_{\mathrm{rh}} \mathrm{W}\left(\mathscr{D}_{X}, Q\right)$. As before, the construction is functorial for noncharacteristic objects in $\mathrm{MF}_{\mathrm{rh}} \mathrm{W}\left(\mathscr{D}_{Y}, \mathrm{Q}\right)$. A proof similar to the one of 3.4.3 - yields the following:

3.4.9 - Proposition. Let $\mathscr{\mathscr { L }} \in \operatorname{MHM}(Y, \mathrm{Q})$ be a mixed Hodge module and $f: X \rightarrow Y$ a morphism of smooth complex algebraic varieties. Assume that $\mathscr{L}$ is noncharacteristic for $f$. Then, its noncharacteristic inverse image $f_{\mathscr{O}}^{*} \underline{L}$ is a mixed Hodge module on $X$.

3.4.10 - REMARK. If $\mathscr{L}$ is noncharacteristic then we have an isomorphism in the derived category $\mathrm{D}^{\mathrm{b}}(\operatorname{MHM}(X, \mathrm{Q}))$

$$
f_{\mathscr{O}}^{*} \underline{\mathscr{L C}} \simeq f^{*} \underline{\mathscr{L C}}[d]
$$

where $f^{*} \mathscr{L}$ is the pullback functor constructed in $[19,4.4]$. 


\section{Mixed Hodge $\mathscr{D}$-complexes}

\section{1 - Categories of diagrams}

4.1.1 - If $R$ is one of the symbols $\mathrm{Q}, \mathrm{C}$ or $\mathscr{D}$ we let $\mathscr{C}_{R}$ be an additive category. We assume to be given on the homotopy category $\mathrm{K}^{\mathrm{b}}\left(\mathscr{C}_{R}\right)$ a null system $N_{R}$ and one denotes by $\mathcal{Q}_{R}$ the associated multiplicative system.

4.1.2 - We also assume to be given a full DG-subcategory $\mathbf{C}_{R}$ of $\left(\mathscr{C}_{R}\right)$. As usual one denotes by

$$
\mathrm{C}_{R}=\mathrm{Z}^{0} \mathrm{C}_{R} \quad \mathrm{~K}_{R}:=\mathrm{H}^{0} \mathbf{C}_{R}
$$

the associated category and the homotopy category, which are respectively full subcategories of $\mathrm{C}^{\mathrm{b}}\left(\mathscr{C}_{R}\right)$ and $\mathrm{K}^{\mathrm{b}}\left(\mathscr{C}_{R}\right)$. We assume that the objects of $\mathbf{C}_{R}$ enjoy the following stability conditions:

- if $a: A \rightarrow A^{\prime}$ is a morphism in $\mathrm{C}^{\mathrm{b}}\left(\mathscr{C}_{R}\right)$ which belongs to $\mathcal{Q}_{R}$, then $A$ belongs to $\mathbf{C}_{R}$ if and only if $A^{\prime}$ belongs to $\mathbf{C}_{R}$;

- if $a: A \rightarrow A^{\prime}$ is a morphism in $\mathrm{C}_{R}$ then its mapping cone is also an object in $\mathbf{C}_{R}$, in particular if $A \in \mathrm{C}_{R}$ then $A[1] \in \mathrm{C}_{R}$.

These conditions ensure that $\mathrm{K}_{R}$ is a strictly full triangulated subcategory of $\mathrm{K}^{\mathrm{b}}\left(\mathscr{C}_{R}\right)$ and that $\mathrm{D}_{R}:=\mathrm{K}_{R}\left[\mathcal{Q}_{R}^{-1}\right]$ is also strictly full triangulated subcategory of $\mathrm{K}^{\mathrm{b}}\left(\mathscr{C}_{R}\right)\left[\mathcal{Q}_{R}^{-1}\right]$ We also assume given two DG-functors

$$
\Phi_{R}: \mathbf{C}_{R} \rightarrow \mathbf{C}_{\mathrm{C}} \quad R=\mathrm{Q}, \mathscr{D}
$$

such that $\Phi_{R}\left(\mathcal{Q}_{R}\right) \subset \mathcal{Q}_{\mathrm{C}}$ and $\Phi_{R}(\operatorname{Mc}(a))=\operatorname{Mc}\left(\Phi_{R}(a)\right)$ for $a \in\left[A, A^{\prime}\right]$. In particular we have induced functors

$$
\Phi_{R}: \mathrm{K}_{R} \rightarrow \mathrm{K}_{\mathrm{C}} \quad \Phi_{R}: \mathrm{D}_{R} \rightarrow \mathrm{D}_{\mathrm{C}}
$$

which are triangulated.

4.1.3 - Definition. A diagram of the form

$$
\underline{A}=\left(\begin{array}{cccc}
\alpha_{\mathbb{Q}}^{A} & A_{\mathbb{C}} & & \\
& & & \\
A_{\mathbb{Q}} & & & A_{\mathscr{D}}
\end{array}\right)
$$

consists then of the following data:

- for $R=\mathrm{Q}, \mathrm{C}, \mathscr{D}$ an object $A_{R}$ in $\mathrm{C}_{R}$; 
- two morphism in $\mathrm{C}_{\mathrm{C}}$

$$
\alpha_{R}^{A}: \Phi_{R}\left(A_{R}\right) \rightarrow A_{\mathrm{C}} \quad R=\mathrm{Q}, \mathscr{D}
$$

which belongs to $\mathcal{Q}_{\mathrm{C}}$.

4.1.4 - The complex of morphisms between two diagrams $\underline{A}$ and $\underline{A}^{\prime}$ is defined by

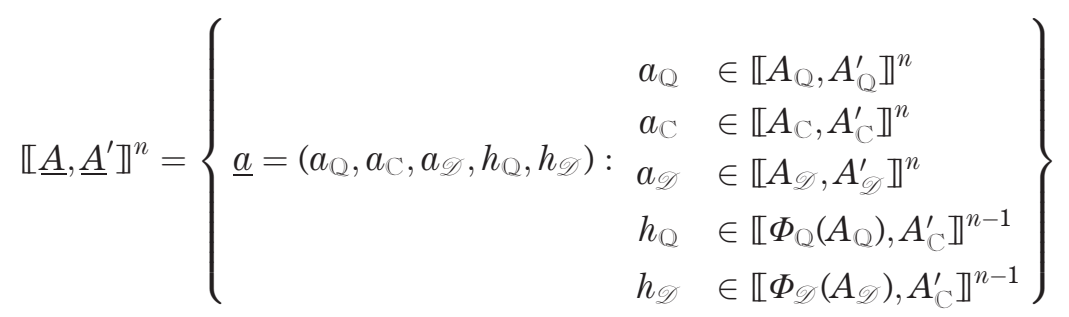

where the differential is given by

$$
\delta\left(\begin{array}{c}
a_{\mathrm{Q}} \\
a_{\mathrm{C}} \\
a_{\mathscr{Q}} \\
h_{\mathrm{Q}} \\
h_{\mathscr{Q}}
\end{array}\right)=\left(\begin{array}{c}
\delta a_{\mathrm{Q}} \\
\delta a_{\mathbb{C}} \\
\delta a_{\mathscr{Q}} \\
a_{\mathrm{C}} \circ \alpha \frac{A}{\mathrm{Q}}-\alpha \frac{A^{\prime}}{\mathrm{Q}} \circ \Phi_{\mathrm{Q}}\left(a_{\mathrm{Q}}\right)-\delta h_{\mathrm{Q}} \\
a_{\mathrm{C}} \circ \alpha \frac{A}{\mathscr{D}}-\alpha \frac{A^{\prime}}{\mathscr{D}} \circ \Phi_{\mathscr{D}}\left(a_{\mathscr{Q}}\right)-\delta h_{\mathscr{D}}
\end{array}\right) .
$$

We get a DG-category $\mathbf{C}_{\mathscr{Q}, Q}$ and we denote by

$$
\mathrm{C}_{\mathscr{D}, \mathrm{Q}}=Z^{0} \mathbf{C}_{\mathscr{D}, \mathrm{Q}} \quad \mathrm{K}_{\mathscr{D}, \mathrm{Q}}=\mathrm{H}^{0} \mathbf{C}_{\mathscr{D}, \mathrm{Q}}
$$

the associated categories. A morphism $\underline{a}=\left(a_{\mathrm{Q}}, a_{\mathrm{C}}, a_{\mathscr{V}}, h_{\mathrm{Q}}, h_{\mathrm{C}}\right)$ in $\mathrm{C}_{\mathscr{D}, \mathrm{Q}}$ is thus a 5-uplet such that $u_{R}$ is a morphism of complexes and $h_{\mathrm{Q}}, h_{\mathscr{D}}$ are graded morphisms of degree -1 whose differentials are equal to the defect in commutativity of the squares

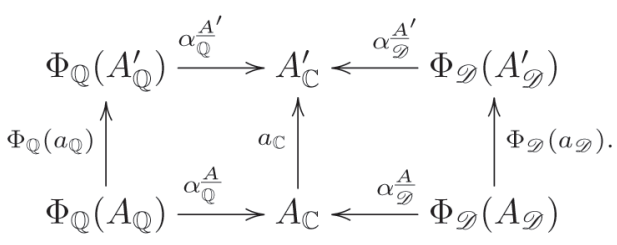

4.1.5 - By the definition of the mapping cone, the forgetful functors 


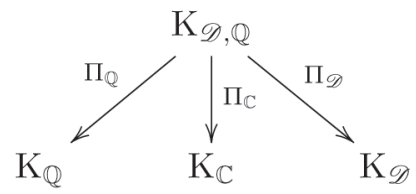

are triangulated. Let $N_{\mathscr{Q}, \mathrm{Q}}$ be the set of diagrams $\underline{A}$ which have their components $A_{R}$ in $N_{R}$. Then $N_{\mathscr{Q}, \mathrm{Q}}$ is a null system in the triangulated category $\mathrm{K}_{\mathscr{O}, \mathcal{Q}}$ and the associated multiplicative system $\mathcal{Q}_{\mathscr{Q}, \mathcal{Q}}$ consists of the morphisms $\underline{a}: \underline{A} \rightarrow \underline{A}^{\prime}$ in $\mathrm{K}_{\mathscr{Q}, \mathbb{Q}}$ such that $a_{R}$ belongs to $\mathcal{Q}_{R}$. The triangulated category $\mathrm{D}_{\mathscr{D}, \mathrm{Q}}$ is defined as the localization of $\mathrm{K}_{\mathscr{O}, \mathrm{Q}}$ with respect to the multiplicative system $\mathcal{Q}_{\mathscr{Q}, \mathrm{Q}}$ :

$$
\mathrm{D}_{\mathscr{Q}, \mathrm{Q}}:=\mathrm{K}_{\mathscr{D}, \mathrm{Q}}\left[\mathcal{Q}_{\mathscr{D}, \mathrm{Q}}^{-1}\right] .
$$

\section{2 - Pure Q-Hodge complexes}

4.2.1 - Let $R=\mathrm{Q}, \mathrm{C}$, one denotes by $\mathbf{C}_{\mathrm{c}}^{+}(X, R)^{\mathrm{b}}$ the DG-category of complexes of sheaves of $R$-vector spaces $K$ on $X^{\text {an }}$ such that

- $K$ is bounded below;

- the cohomology groups $\mathrm{H}^{i}(K)$ are algebraically constructible for all $i \in Z$ and nonzero for finitely many $i$ 's.

4.2.2 - Recall that $\mathrm{C}_{\mathrm{rh}}^{\mathrm{b}} \mathrm{F}\left(\mathscr{D}_{X}, \mathrm{Q}\right)$ is the category of diagrams of the shape (see [9, 3.1.4] for details)

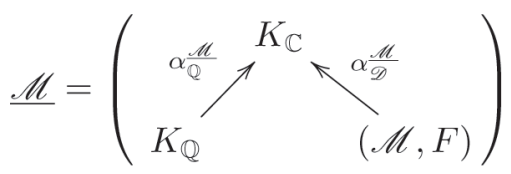

where for $R=\mathrm{Q}, \mathrm{C}, K_{R}$ is an object in $\mathrm{C}_{\mathrm{c}}^{*}\left(X^{\text {an }}, R\right)^{\mathrm{b}}$ while $(\mathscr{l}, F)$ is an object in $\mathrm{C}_{\mathrm{rh}}^{\mathrm{b}} \mathrm{F}\left(\mathscr{D}_{X}\right)$. The comparison morphisms are quasi-isomorphisms:

$\alpha \frac{\mathscr{K C}}{\mathrm{Q}}: \Phi_{\mathrm{Q}}\left(K_{\mathrm{Q}}\right):=K_{\mathrm{Q}} \otimes_{\mathrm{Q}} \otimes \mathrm{C} \rightarrow K_{\mathrm{C}} \quad \alpha \frac{\mathscr{K}}{\mathscr{D}}: \Phi_{\mathscr{D}}(\mathscr{C l}):=\operatorname{Sp}_{\mathrm{an}}(\mathscr{C O}) \rightarrow K_{\mathrm{C}}$.

4.2.3 - The triangulated category $\mathrm{D}_{\mathrm{rh}}^{\mathrm{b}} \mathrm{F}\left(\mathscr{D}_{X}, \mathrm{Q}\right)$, obtained from $\mathrm{C}_{\mathrm{rh}}^{\mathrm{b}} \mathrm{F}\left(\mathscr{D}_{X}, Q\right)$ as in 4.1 .5 -, has a natural $t$-structure [9, 3.2.2] and we denote by ${ }^{\mathrm{P}} \underline{H}^{i}$ the associated cohomological functors. Given an integer $w \in Z$, the category $\mathrm{D}_{\mathscr{H}}^{b}(X, Q, w)_{\mathscr{D}}$ of pure Hodge $\mathscr{D}$-complexes of weight $w$ is defined as the strictly full subcategory of $\mathrm{D}_{\mathrm{rh}}^{\mathrm{b}} \mathrm{F}\left(\mathscr{D}_{X}, \mathrm{Q}\right)$ formed by the objects $\mathscr{C}$ that satisfy the following two conditions:

- (weight $w$ condition) for every $i \in Z,{ }^{\mathrm{P}} \underline{H}^{i}(\mathscr{L C})$ is a pure Q-Hodge module of weight $i+w$, i.e. lies in $\operatorname{MH}(X, i+w)$; 
- (decomposability condition) there exists an isomorphism in $\mathrm{D}_{\mathrm{rh}}^{\mathrm{b}} \mathrm{F}\left(\mathscr{D}_{X} ; \mathrm{Q}\right)$

$$
\underline{\mathscr{C}} \simeq \bigoplus_{i \in Z}^{\mathrm{P}} \underline{\mathrm{H}}^{i}(\underline{\mathscr{L}})[-i]
$$

\section{3 - Mixed Q-Hodge complexes}

4.3.1 - Given an increasing filtration $W$ and an integer $n \in Z$, recall that $W\{n\}$ is the filtration $W\{n\}_{k}=W_{k-n}$. For $R=\mathrm{Q}$, C one denotes by $\mathrm{C}^{+}(X, R ; W)^{\mathrm{b}}$ the category of complexes of sheaves of $R$-vector spaces on $X^{\text {an }}$ endowed with a biregular increasing filtration $W$ and satisfying some boundedness conditions. Let $\mathrm{C}_{\mathrm{c}}^{+}(X, R ; W)^{\mathrm{b}}$ be the full subcategory of it formed by the complexes $(K, W)$ such that

$$
\mathrm{Gr}_{n}^{W} K \in \mathrm{C}_{\mathrm{c}}^{+}(X, R) .
$$

We set $\mathrm{C}^{*}(X ; W)_{R}^{\mathrm{b}}:=\mathrm{C}_{\mathrm{c}}^{*}(X, R ; W)^{\mathrm{b}}$.

4.3.2 - Consider now the subcategory $\mathrm{C}_{\mathrm{rh}}^{\mathrm{b}} \mathrm{F}\left(\mathscr{D}_{X} ; W\right)$ of the category of complexes $\mathrm{C}^{\mathrm{b}}\left(\operatorname{MF}\left(\mathscr{D}_{X} ; W\right)\right)$ formed by the complexes such that $\left({ }^{6}\right)$

$$
\mathrm{Gr}_{n}^{W} K_{\mathscr{D}} \in \mathrm{C}_{\mathrm{rh}}^{\mathrm{b}} \mathrm{F}\left(\mathscr{D}_{X}\right)
$$

for any $n \in Z$ Z. We set $\mathrm{C}^{*}(X ; W)_{\mathscr{D}}^{\mathrm{b}}:=\mathrm{C}_{\mathrm{rh}}^{\mathrm{b}} \mathrm{F}\left(\mathscr{D}_{X} ; W\right)$.

4.3.3 - Let $R=$ Q, C, $\mathscr{D}$ and $u_{R}:\left(K_{R}, W_{R}\right) \rightarrow\left(L_{R}, W_{R}\right)$ be a morphism in the category $\mathrm{C}^{*}(X ; W)_{R}^{\mathrm{b}}$, the mixed cone of $u_{R}$ is defined by

$$
W_{n} \operatorname{Mc}\left(u_{R}\right)_{\mathscr{H}}=W_{R, n} L_{R}^{n} \oplus W_{R, n-1} K_{R}^{n+1}
$$

with the usual differential of a mapping cone. In particular if $\left(L_{R}, W_{R}\right)=0$ we get the (mixed) shift functor defined by

$$
\left(K_{R}, W_{R}\right)\{1\}:=\left(K_{R}[1], W_{R}\{1\}\right) .
$$

4.3.4 - Let $\mathrm{C}_{\mathrm{rh}}^{\mathrm{b}} \mathrm{F}\left(\mathscr{D}_{X}, \mathrm{Q} ; W\right)$ be the category of diagrams of the shape

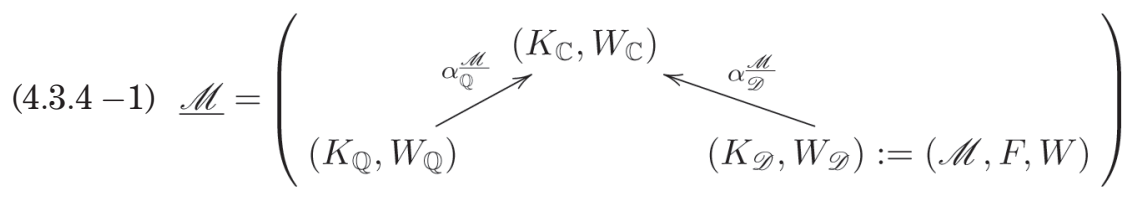

$\left({ }^{6}\right)$ For any $n \in Z$, we denote by $W_{n} K_{\mathscr{D}}$ and $\mathrm{Gr}_{n}^{W} K_{\mathscr{D}}$ the right $\mathscr{D}{ }_{X}$-modules $W_{n} \mathscr{L} 6$ and $\mathrm{Gr}_{n}^{W} \mathscr{C l}$ endowed with the filtration induced by $F$. 
where $\left(K_{R}, W_{R}\right)$ is an object in $\mathrm{C}^{*}(X ; W)_{R}^{\mathrm{b}}$ for $R=\mathrm{Q}, \mathrm{C}$ and $\left(K_{\mathscr{D}}, W_{\mathscr{D}}\right):=$ $(\mathscr{l}, F, W)$ is an object in $\mathrm{C}_{\mathrm{rh}}^{\mathrm{b}} \mathrm{F}\left(\mathscr{D}_{X} ; W\right)$. The comparison morphisms

$$
\alpha \frac{\mathscr{H}}{\mathrm{Q}}:\left(K_{\mathrm{Q}}, W_{\mathrm{Q}}\right) \otimes \mathbb{C} \rightarrow\left(K_{\mathbb{C}}, W_{\mathrm{C}}\right) \quad \alpha \frac{\mathscr{L l}}{\mathscr{D}}: \operatorname{Sp}_{\mathrm{an}}(\mathscr{L l}, W) \rightarrow\left(K_{\mathrm{C}}, W_{\mathrm{C}}\right)
$$

are filtered quasi-isomorphisms with respect to the weight filtration.

4.3.5 - The category of mixed Hodge complexes is built on a less stringent homotopy relation that the one for general diagrams. A morphism $\underline{u}$ in $\mathrm{C}_{\mathrm{rh}}^{\mathrm{b}} \mathrm{F}\left(\mathscr{D}_{X}, \mathrm{Q} ; W\right)$ is homotopic to zero iff there exists a 5-uplet $\underline{v}:=$ $\left(v_{\mathrm{Q}}, v_{\mathrm{C}}, v_{\mathscr{O}}, k_{\mathrm{Q}}, k_{\mathscr{D}}\right)$ where

$$
v_{R}:\left(K_{R}, W_{R}\right) \rightarrow\left(K_{R}^{\prime}, W_{R}^{\prime}\{-1\}\right)
$$

are morphism of degree -1 in $\mathbf{C}^{*}(X ; W)_{R}^{b}$ and

$$
k_{R}: \Phi_{R}\left(K_{R}, W_{R}\right) \rightarrow\left(K_{R}^{\prime}, W_{R}^{\prime}\{-1\}\right)
$$

is a morphism of degree -2 in $\mathbf{C}^{*}(X ; W)_{\mathrm{C}}^{\mathrm{b}}$ such that

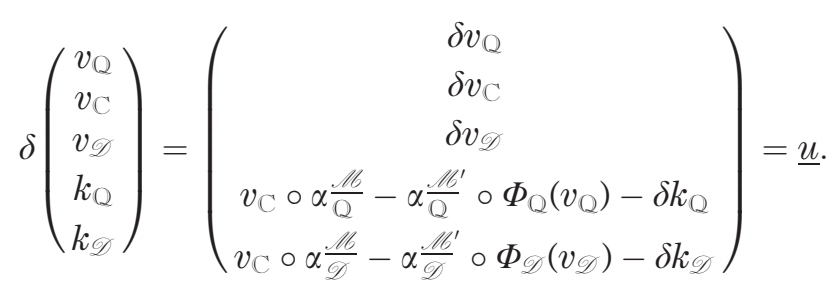

holds.

4.3.6 - Let us denote by $\mathrm{K}_{\mathrm{rh}}^{\mathrm{b}} \mathrm{FW}(\mathscr{D} \mathrm{x}, \mathrm{Q})$ the category obtained from the diagram category $\mathrm{C}_{\mathrm{rh}}^{\mathrm{b}} \mathrm{F}\left(\mathscr{D}_{X}, \mathrm{Q} ; W\right)$ by killing the morphisms homotopic to zero in this sense. If one defines distinguished triangles in this category using this mixed cone and the shift functor $(4.3 .3-1)$, we get a triangulated structure.

By construction we have a well defined functors

$$
\mathrm{Gr}_{n}^{W}: \mathrm{K}_{\mathrm{rh}}^{\mathrm{b}} \mathrm{FW}(\mathscr{D} \mathrm{x}, \mathrm{Q}) \rightarrow \mathrm{D}_{\mathrm{rh}}^{\mathrm{b}} \mathrm{F}(\mathscr{D} \mathrm{x}, Q) .
$$

The category $\mathrm{K}_{\mathscr{H}}^{\mathrm{b}}(X, \mathrm{Q})_{\mathscr{Q}}$ of mixed $\mathrm{Q}$-Hodge $\mathscr{D}$-complexes is the full subcategory of $\mathrm{K}_{\mathrm{rh}}^{\mathrm{b}} \mathrm{FW}(\mathscr{D} \mathrm{X}, \mathrm{Q})$ formed by the diagrams $\mathscr{\mathscr { C }}$ such that

$$
\operatorname{Gr}_{n}^{W} \underline{\mathscr{l}} \in \mathrm{D}_{\mathscr{H}}^{\mathrm{b}}(X, \mathrm{Q}, n)_{\mathscr{D}}
$$

for any integer $n \in Z$. 
Let $\operatorname{Mc}(\underline{u})_{\mathscr{H}}$ be the mixed cone of a morphism $\underline{u}: \underline{\mathscr{C}} \rightarrow \underline{\mathscr{C}^{\prime}}$. Then by definition we have a decomposition as a direct sum of complexes

$$
\operatorname{Gr}_{n}^{W}\left(\operatorname{Mc}(\underline{u})_{\mathscr{B}}\right)=\operatorname{Gr}_{n}^{W}\left(\underline{\mathscr{C}^{\prime}}\right) \oplus \operatorname{Gr}_{n-1}^{W}(\underline{\mathscr{C}})[1]
$$

Since both direct summands are pure Hodge complexes of weight $n$, this shows that the mixed cone of a morphism of mixed Hodge complexes is still a mixed Hodge complex. A Betti quasi-isomorphism in $\mathrm{K}_{\mathscr{C}}^{\mathrm{b}}(X, \mathrm{Q})_{\mathscr{Q}}$ is a morphism $\underline{u}$ such that $u_{\mathrm{Q}}$ is a quasi-isomorphism. The set $\mathcal{Q}_{\mathscr{H}}$ of Betti quasi-isomorphisms forms a multiplicative system in $\mathrm{K}_{\mathscr{G}}^{\mathrm{b}}(X, Q)_{\mathscr{Q}}$ and the category of mixed Hodge $\mathscr{D}$-complexes is defined as the localization:

$$
\mathrm{D}_{\mathscr{H}}^{\mathrm{b}}(X, Q)_{\mathscr{D}}:=\mathrm{K}_{\mathscr{H}}^{\mathrm{b}}(X, Q)_{\mathscr{O}}\left[\mathcal{Q}_{\mathscr{H}}^{-1}\right] \text {. }
$$

4.3.7 - Recall that the realization functor of $[9,5.3]$

$$
\text { real : } \mathrm{D}^{\mathrm{b}}(\operatorname{MHM}(X, \mathrm{Q})) \rightarrow \mathrm{D}_{\mathscr{H}}^{\mathrm{b}}(X, \mathrm{Q})_{\mathscr{D}}
$$

is obtained via two functors (see [9, 5.3.1-2] for details)

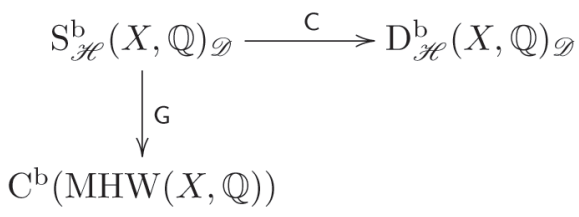

where $\mathrm{S}_{\mathscr{H}}^{\mathrm{b}}(X, \mathrm{Q})_{\mathscr{D}}$ denotes the category of stupid mixed Q-Hodge $\mathscr{D}$ complexes as defined in $[9,5.3 .1]$

4.3.8 - It will be convenient in the sequel to consider also the triangulated category $\mathrm{D}_{\mathscr{H}}^{\mathrm{b}}(X, Q)_{\mathscr{D}}^{\mathrm{L}}$ of left mixed Hodge complexes obtained by a similar construction using left $\mathscr{D}$-modules instead of right $\mathscr{D}$-modules and the analytic de Rham functor $\mathrm{DR}_{\mathrm{an}}$ for glueing instead of the analytic Spencer complex. The side changing functors induce an equivalence of triangulated categories between $\mathrm{D}_{\mathscr{H}}^{b}(X, Q) \mathscr{Q}$ and $\mathrm{D}_{\mathscr{H}}^{b}(X, Q)_{\mathscr{D}}^{\mathrm{L}}$.

4.3.9 - With an admissible variation of mixed Hodge structures $\underline{V}$ on $X$ we can associate a left mixed Hodge $\mathscr{D}$-complex

$$
\mathscr{H}_{\mathbb{V}}:=(\overbrace{\left(\mathbb{V}_{\mathbb{Q}}, W_{\mathbb{Q}}\right)}^{\alpha_{\frac{\mathbb{V}}{\mathbb{V}} \operatorname{DR}_{\text {an }}(\mathscr{V}, W)}})
$$


where $\alpha \frac{\mathrm{V}}{\mathrm{Q}}$ denotes the composition:

$$
\left(\mathbb{V}_{\mathrm{C}}, W_{\mathrm{C}}\right) \stackrel{\alpha \underline{\underline{Y}}}{\longrightarrow}\left(\operatorname{ker} \nabla^{\mathrm{an}}, W\right) \hookrightarrow \mathrm{DR}_{\mathrm{an}}(\mathscr{V}, W) .
$$

The associated right mixed Hodge complex is

$$
\underline{\mathbb{V}}^{\mathscr{H}}:=(\overbrace{\left(\mathbb{V}_{\mathbb{Q}}, W_{\mathbb{Q}}\right)\left\{d_{X}\right\}}^{\alpha_{\mathbb{Q}}^{\underline{\mathbb{V}}} \operatorname{Sp}_{\text {an }}(\mathscr{V}, W)^{r} \text { Id }} \underbrace{}_{(\mathscr{V}, F, W)^{r}})
$$

where one uses the isomorphism of filtered complexes

$$
\operatorname{Sp}_{\text {an }}(\mathscr{V}, W)^{r} \simeq \operatorname{DR}_{\text {an }}(\mathscr{V}, W)\left\{d_{X}\right\}
$$

to define $\alpha \frac{\mathrm{V}}{\mathrm{Q}}$. The diagram $(4.3 .9-2)$ is a mixed Hodge $\mathscr{D}$-complex since for all $n \in \mathbb{Z} \backslash\{0\}$

$$
\underline{\mathrm{P}}^{i}\left(\mathrm{Gr}_{n}^{W_{\underline{Y}}^{\mathscr{B}}}\right)=0
$$

and ${ }^{\mathrm{P}} \underline{\mathrm{H}}^{0}\left(\mathrm{Gr}_{n}^{W} \underline{\mathrm{V}}^{\mathscr{H}}\right)$ is the pure Hodge module of weight $n$ associated with the pure variation $\mathrm{Gr}_{n-d_{X}}^{W} \underline{\mathrm{V}}$. Therefore, the two conditions in 4.2.2 - are satisfied.

4.3.10 - For technical reasons, we will have to consider the category $\mathrm{D}_{\mathscr{G}}^{\mathrm{b}}(X, \mathrm{Q})_{\mathscr{D}}^{r}$ of mixed Q-Hodge $\mathscr{D}$-complexes of length $r \geqslant 1$. Let us fix now our convention. If $r \geqslant 0$ we denote by Ex, the functor

$$
\mathrm{Ex}:=\operatorname{Ex}_{r}^{r}: \mathrm{D}_{\mathscr{H}}^{\mathrm{b}}(X, Q)_{\mathscr{Q}}^{r} \rightarrow \mathrm{D}_{\mathscr{H}}^{\mathrm{b}}(X, Q)_{\mathscr{Q}}^{r+1} .
$$

as defined in [9, 4.2.2]. Similarly, for $r \geqslant 1$, one denotes by Co the functor

$$
\mathrm{Co}:=\mathrm{Co}_{r-1}^{r}: \mathrm{D}_{\mathscr{H}}^{\mathrm{b}}(X, \mathrm{Q})_{\mathscr{Q}}^{r} \rightarrow \mathrm{D}_{\mathscr{H}}^{\mathrm{b}}(X, \mathrm{Q})_{\mathscr{Q}}^{r-1}
$$

as defined in [9, 4.2.2].

\section{Proof of the main theorem}

The proof of the main theorem uses an external product and a noncharacteristic pullback for mixed Q-Hodge complexes. As the construction of these operations and the verification of their properties are not difficult but nonetheless quite technical, we will postpone the details until the next section. 
Let $X$ and $Y$ be two smooth complex algebraic varieties and $\mathscr{L}, \mathscr{N}$ be mixed Q-Hodge complexes on $X$ and $Y$. We denote by $\mathscr{L} \otimes \cdot \mathscr{N}$ the external product of $\mathscr{L}$ and $\mathscr{N}$ which is a mixed Q-Hodge complex on $X \times Y$ (see 6.5.9). If $\mathscr{N}$ is noncharacteristic with respect to a morphism $f: X \rightarrow Y$, then we denote by $f_{\mathscr{Q}}^{*} \mathscr{\mathscr { C }}$ the pullback of $\mathscr{N}$ defined in $6.3-$.

\section{1 - Module structure over a tensor category}

5.1.1 - Let $(\mathrm{C}, \otimes, 1)$ be a monoidal category and $\mathrm{M}$ a category. A module structure on $\mathrm{M}$ is the data of triple $(\circledast, \alpha, \mu)$ where

$$
\mathrm{C} \times \mathrm{M} \rightarrow \mathrm{M} ;(\mathscr{C}, \mathscr{C}) \mapsto \mathscr{C} \circledast \mathscr{C}
$$

is a bifunctor,

$$
\alpha_{\mathscr{X}, \mathscr{Y}, \mathscr{C}}: \mathscr{X} \circledast(\mathscr{Y} \circledast \mathscr{C}) \rightarrow(\mathscr{K} \otimes \mathscr{Y}) \circledast \mathscr{C}
$$

is an isomorphism functorial in $\mathscr{B}, \mathscr{Z} \in \mathrm{C}, \mathscr{C l} \in \mathrm{M}$ and $\mu_{\mathscr{C}}: \mathbb{1} \circledast \mathscr{C} b \rightarrow \mathscr{C}$ is an isomorphism functorial in $\mathscr{L} \in \mathrm{M}$. These data are assumed to satisfy the following coherence conditions:

- (pentagonal identity) the pentagon

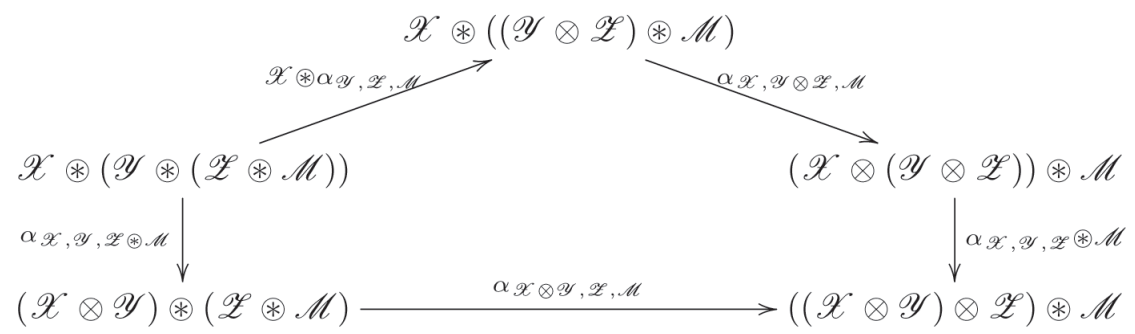

is commutative where $\alpha_{\mathscr{6}, \mathscr{Y}, \mathscr{E}}$ is the associativity constraint in C.

- the triangles
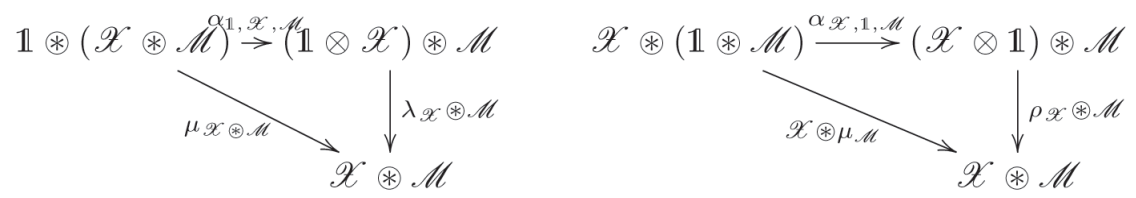

are commutative where $\lambda_{\mathscr{C}}$ and $\rho_{\mathscr{C}}$ are the unit isomorphisms in C. 
5.1.2 - A morphism of modules $(\mathrm{M}, \circledast) \rightarrow(\mathrm{N}, \circledast)$ is a pair $(\mathrm{F}, \Upsilon)$ where $\mathrm{F}: \mathrm{M} \rightarrow \mathrm{N}$ is a functor and

$$
\Upsilon_{\mathscr{X}, \mathscr{C O}}: \mathscr{C} \circledast \mathrm{F}(\mathscr{C l}) \rightarrow \mathrm{F}(\mathscr{B} \circledast \mathscr{C l})
$$

is an isomorphism functorial in $\mathscr{C} \in \mathrm{C}$ and $\mathscr{C} \in \mathrm{M}$ such that

$(5.1 .2-1)$

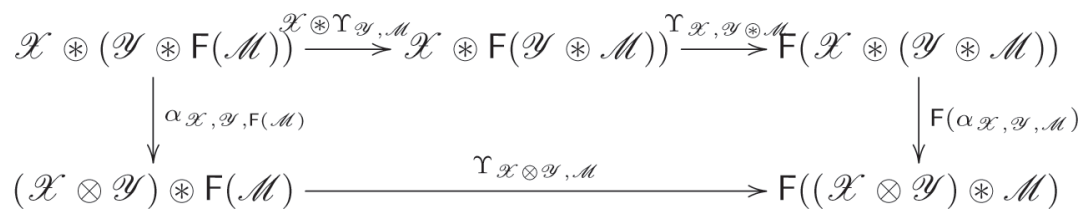

$(5.1 .2-2)$

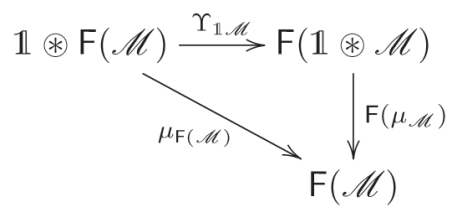

are commutative.

5.1.3 - Let $(\mathrm{C}, \otimes, 1)$ be a monoidal category. Given an object $\mathscr{B}$ in $\mathrm{C}$, a dual is triple ( $\mathscr{H}$, ev, coev) where $\mathscr{H}$ is an object in $\mathrm{C}$ and

$$
\text { ev }: \mathscr{C} \otimes \mathscr{H} \rightarrow 1 \quad \text { coev }: 1 \rightarrow \mathscr{H} \otimes \mathscr{C}
$$

are morphisms in $\mathrm{C}$ such that the compositions

$(5.1 .3-1)$

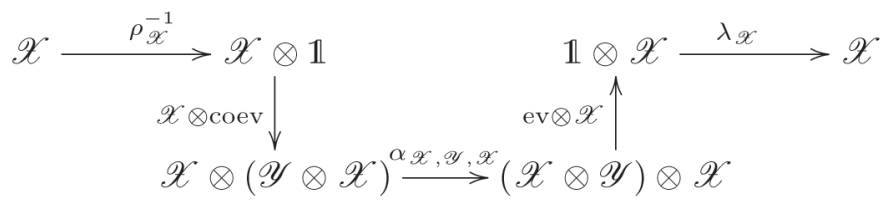

$(5.1 .3-2)$

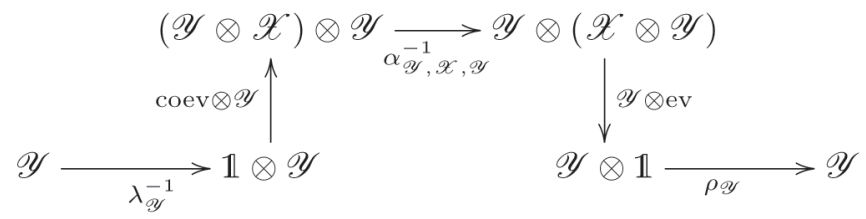


are the identity. Let now $(\mathrm{M}, \circledast)$ be a module over $(\mathrm{C}, \otimes, 1)$ and $\mathscr{C}, \mathscr{N}$ be objects in M. If ( $\mathscr{H}$, ev, coev) is a dual of $\mathscr{C}$ in $\mathrm{C}$ then the morphism

$$
\gamma_{\mathscr{X}, \mathscr{L}, \mathscr{N}}: \operatorname{Hom}_{\mathrm{M}}(\mathscr{C} \circledast \mathscr{C}, \mathscr{N}) \rightarrow \operatorname{Hom} \mathrm{M}(\mathscr{L}, \mathscr{H} \circledast \mathscr{N})
$$

which maps $\phi: \mathscr{B} \circledast \mathscr{C} \rightarrow \mathscr{N}$ to the composition

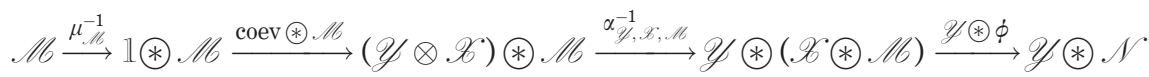

is an isomorphism. Indeed since (5.1.3-1) and (5.1.3-2) are the identity, the morphism

$$
\delta_{\mathscr{X}}, \mathscr{C l}, \mathscr{N}: \operatorname{Hom}_{\mathrm{M}}(\mathscr{C l}, \mathscr{Y} \circledast \mathscr{N}) \rightarrow \operatorname{Hom}_{\mathrm{M}}(\mathscr{C} \circledast \mathscr{C}, \mathscr{N})
$$

which maps $\psi: \mathscr{C l} \rightarrow \mathscr{Y} \circledast \mathscr{N}$ to the composition

$\mathscr{C} \circledast \mathscr{L} \stackrel{\mathscr{C} \circledast \psi}{\longrightarrow} \mathscr{C} \circledast(\mathscr{H} \circledast \mathscr{N}) \stackrel{\alpha \mathscr{Y}, \mathscr{N}}{\longrightarrow}(\mathscr{C} \otimes \mathscr{H}) \circledast \mathscr{N} \stackrel{\mathrm{ev} \circledast \mathscr{N}}{\longrightarrow} \mathbb{1} \circledast \mathscr{N} \stackrel{\mu \mathscr{N}}{\longrightarrow} \mathscr{N}$

is inverse to $\gamma_{\mathscr{C}, \mathscr{L}, \mathscr{N}}$.

5.1.4 - REMARK. Let $(\mathrm{M}, \circledast)$ and $(\mathrm{N}, \circledast)$ be two modules over $(\mathrm{C}, \otimes, 1)$. If $(\mathrm{F}, \Upsilon):(\mathrm{M}, \circledast) \rightarrow(\mathrm{N}, \circledast)$ is a morphism of modules, then it follows from the functoriality of the maps $\Upsilon_{\mathscr{X}, \mathscr{C}}$ and basic coherence diagrams $(5.1 .2-1),(5.1 .2-2)$ that

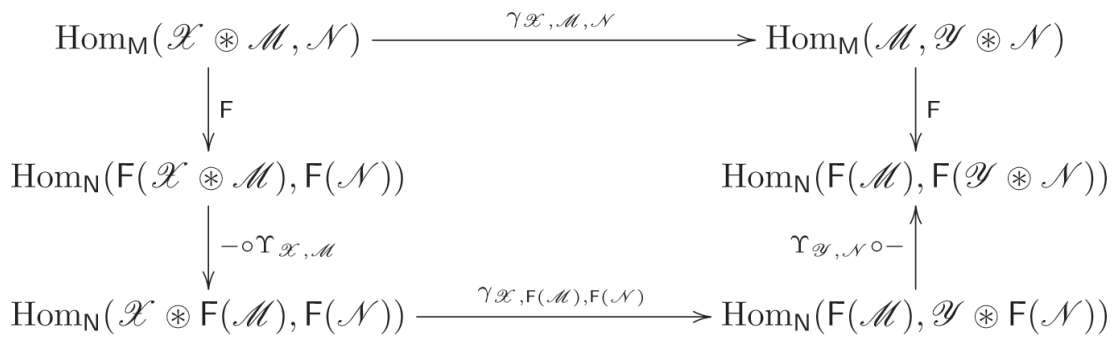

is a commutative diagram.

\section{2 - Tensor product with variations}

5.2.1 - Let $X$ be a smooth complex algebraic variety and $\underline{V}$ an admissible variation of mixed Hodge structures on $X$. By 2.3.2 - the $\mathscr{D}_{X \times X}$-module $\mathscr{T} \otimes \mathscr{C}$ is noncharacteristic with respect to $\Delta_{X}$. Moreover for any filtered right $\mathscr{D}$-module $(\mathscr{C}, F, W)$ endowed with an increasing filtration $W$ by $\mathscr{D} X^{-}$ 
submodules, we have

$$
\operatorname{Gr}_{k}^{F} \operatorname{Gr}_{n}^{W}(\mathscr{V} \otimes \mathscr{C})=\bigoplus_{\substack{n_{1}+n_{2}=n \\ k_{1}+k_{2}=k}}\left(\operatorname{Gr}_{k_{1}}^{F} \mathrm{Gr}_{n_{1}}^{W} \mathscr{\mathscr { V }}\right) \otimes\left(\operatorname{Gr}_{k_{2}}^{F} \mathrm{Gr}_{n_{2}}^{W} \mathscr{\mathscr { C }}\right)
$$

and thus 2.3.2 - implies that

$$
\mathscr{T} 0 r_{i}\left(\operatorname{Gr}_{k}^{F} \operatorname{Gr}_{n}^{W}(\mathscr{V} \otimes \mathscr{C l}), \mathscr{O}_{X}\right)=0 \quad \forall i>0 .
$$

5.2.2 - Let $\underline{\mathscr{C}} \in \mathrm{D}_{\mathscr{H}}^{\mathrm{b}}(X, Q)_{\mathscr{D}}$ a mixed Hodge complex. Then $\underline{\mathrm{V}}^{\mathscr{H}} \mathrm{\square} \underline{\mathscr{C l}}$ is noncharacteristic with respect to the diagonal immersion $\Delta_{X}: X \hookrightarrow X \times X$ in the sense of 6.1.4 -. Indeed since $\nabla$ is an exact bifunctor for the perverse $t$-structure on $\mathrm{D}_{\mathrm{rh}}^{\mathrm{b}} \mathrm{F}\left(\mathscr{D}_{X}, \mathrm{Q}\right)$, it follows that one has an isomorphism

$$
\begin{aligned}
& \underline{\mathrm{P}}^{\mathrm{H}}\left[\mathrm{Gr}_{n}^{W}\left(\underline{\mathbb{V}}^{\mathscr{H}} \otimes \underline{\mathscr{C}}\right)\right]=\underline{\mathrm{P}}^{\mathrm{H}} \underline{\mathrm{H}}^{i}\left[\bigoplus_{n_{1}+n_{2}=n}\left(\mathrm{Gr}_{n_{1}}^{W} \underline{\mathrm{Y}}^{\mathscr{H}}\right) \otimes\left(\mathrm{Gr}_{n_{2}}^{W} \mathscr{\mathscr { C l }}\right)\right] \\
& =\bigoplus_{n_{1}+n_{2}=n} \underline{\mathrm{P}}^{i}\left[\left(\mathrm{Gr}_{n_{1}}^{W} \underline{\mathrm{V}}^{\mathscr{H}}\right) \otimes\left(\mathrm{Gr}_{n_{2}}^{W} \underline{\mathscr{C l}}\right)\right] \\
& =\bigoplus_{n_{1}+n_{2}=n} \mathrm{Gr}_{n_{1}}^{W} \underline{\mathrm{V}}^{\mathscr{H}} \otimes^{\mathrm{P}} \underline{\mathrm{H}}^{i}\left[\mathrm{Gr}_{n_{2}}^{W} \underline{\mathscr{C}}\right]
\end{aligned}
$$

and 5.2.1 - implies that $\underline{\mathrm{V}}^{\mathscr{G}} \otimes \mathscr{\mathscr { C }}$ is noncharacteristic with respect to the diagonal immersion $\Delta_{X}$. Therefore we may define

$$
\underline{V} \circledast \underline{\mathscr{C}}:=\left(\Delta_{X}\right)_{\mathscr{O}}^{*}\left(\underline{\mathrm{V}}^{\mathscr{H}} \otimes \cdot \underline{\mathscr{L C}}\right)
$$

which is a mixed Hodge complex on $X$. This construction is functorial both in $\underline{\mathscr{L}}$ and $\underline{V}$ and we thus get a bifunctor

$$
\circledast: \operatorname{VMHS}(\mathrm{X}, \mathrm{Q})_{\mathrm{ad}} \times \mathrm{D}_{\mathscr{H}}^{\mathrm{b}}(X, Q)_{\mathscr{O}} \rightarrow \mathrm{D}_{\mathscr{H}}^{\mathrm{b}}(X, \mathrm{Q})_{\mathscr{Q}} .
$$

5.2.3 - Proposition. The bifunctor $(5.2 .2-1)$ endows $\mathrm{D}_{\mathscr{H}}^{\mathrm{b}}(X, \mathrm{Q})_{\mathscr{D}}$ with a module structure over the tensor category $\operatorname{VMHS}(\mathrm{X}, \mathrm{Q})_{\mathrm{ad}}$.

Proof. Let us use the following notation:
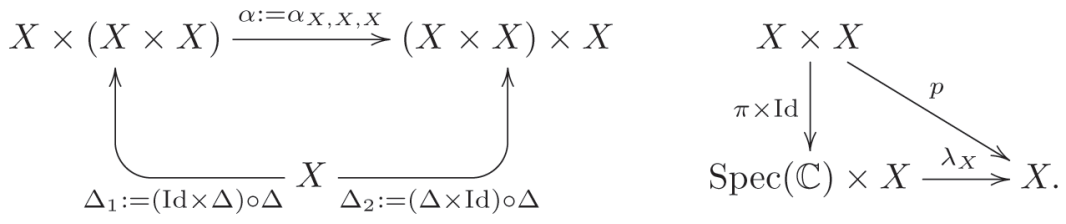
If $\mathbb{W}$ in $\operatorname{VMHS}(\mathrm{X}, \mathrm{Q})_{\mathrm{ad}}$ is another variation, the associativity isomorphism $\alpha_{\underline{\mathrm{V}}, \mathrm{W}, \underline{L}}$ is defined as the composition:

$$
\begin{aligned}
& \underline{\mathbb{V}} \circledast(\underline{\mathbb{W}} \circledast \underline{\mathscr{M}}) \stackrel{c_{\Delta, \mathrm{Id} \times \Delta}^{-1}}{\longrightarrow} \Delta_{1}^{*}\left(\underline{\mathbb{V}}^{\mathscr{H}} \nabla\left(\underline{\mathbb{W}}^{\mathscr{H}} \nabla \underline{\mathscr{M}}\right)\right) \stackrel{{ }_{\Delta_{2}, \alpha}-1}{\longrightarrow} \Delta_{2}^{*}\left(\left(\underline{\mathbb{V}}^{\mathscr{H}} \otimes \underline{\mathbb{W}}^{\mathscr{H}}\right) \nabla \underline{\mathscr{M}}\right)
\end{aligned}
$$

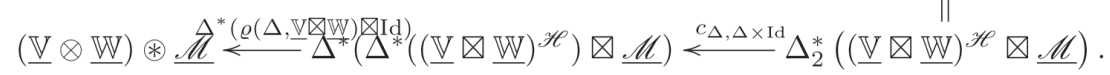

The unit isomorphism is defined as the composition:

$$
\begin{aligned}
\mathbb{Q}_{X}^{\mathscr{H}} \circledast \underline{\mathscr{M}}=\Delta^{*}\left(\mathbb{Q}_{X}^{\mathscr{H}} \otimes \underline{\mathscr{M}}\right) \stackrel{\Delta^{*}\left(\varrho(\pi, \underline{\underline{V}})^{-1} \otimes \mathrm{Id}\right)}{\longrightarrow} & \Delta^{*}\left(\pi^{*} \mathbb{Q}^{\mathscr{H}} \otimes \underline{\mathscr{M}}\right) \\
& c_{\lambda_{X}, \pi \times \mathrm{Id}}^{-1} \downarrow \\
& \Delta^{*} p^{*} \underline{\mathscr{M}} \stackrel{c_{\Delta, p}^{-1}}{\longrightarrow} \underline{\mathscr{M}} .
\end{aligned}
$$

Using the results proved in $6.2-, 6.3-$ and $6.4-$, it is easy to check that these isomorphisms satisfy the relations listed in 5.1.1-.

5.2.4 - Let $\mathscr{L}$ a mixed Hodge module on $X$. By 5.2.1 -, we know that the mixed Hodge module $\underline{V}^{\mathscr{H}} \otimes \cdot \mathscr{\mathscr { C }}$ is noncharacteristic with respect to $\Delta_{X}$. Hence using the pullback defined in 3.4.8-, we may consider given a complex of mixed Hodge modules $\mathscr{\mathscr { C }} \in \mathrm{D}^{\mathrm{b}}(\operatorname{MHM}(X, Q))$, the complex of mixed Hodge modules:

$\left(\Delta_{X}\right)_{\mathscr{O}}^{*}\left(\underline{\mathbb{V}}^{\mathscr{H}} \otimes \underline{\mathscr{C}}\right): \cdots \rightarrow\left(\Delta_{X}\right)_{\mathscr{O}}^{*}\left(\underline{\mathbb{V}}^{\mathscr{H}} \otimes \underline{\mathscr{C}}^{i}\right) \rightarrow\left(\Delta_{X}\right)_{\mathscr{O}}^{*}\left(\underline{\mathbb{V}}^{\mathscr{H}} \otimes \underline{\mathscr{C}}^{i+1}\right) \rightarrow \cdots$.

As for mixed Hodge complexes, one then defines:

$$
\underline{\mathrm{V}} \circledast \underline{\mathscr{L C}}:=\left(\Delta_{X}\right)_{\mathscr{O}}^{*}\left(\underline{\mathrm{V}}^{\mathscr{H}} \otimes \cdot \underline{\mathscr{M C}}\right)
$$

and get a bifunctor

$(5.2 .4-1) \quad \operatorname{VMHS}(\mathrm{X}, \mathrm{Q})_{\mathrm{ad}} \times \mathrm{D}^{\mathrm{b}}(\operatorname{MHM}(X, \mathrm{Q})) \rightarrow \mathrm{D}^{\mathrm{b}}(\operatorname{MHM}(X, \mathrm{Q}))$.

The following lemma is not difficult to check:

5.2.5 - LemMA. The bifunctor (5.2.4-1) endows $\mathrm{D}^{\mathrm{b}}(\mathrm{MHM}(X, \mathrm{Q}))$ with a module structure over the monoidal category $\operatorname{VMHS}(\mathrm{X}, \mathrm{Q})_{\mathrm{ad}}$.

Remark that the same construction works if the category $\operatorname{MHW}(X, \mathrm{Q})$ replaces $\operatorname{MHM}(X, Q)$. 
5.2.6 - REMARK. By 3.4.10 - , for a mixed Hodge module $\mathscr{l l}$ in $\operatorname{MHM}(X, Q)$, we have an isomorphism

$$
\underline{\mathbb{V}} \circledast \underline{\mathscr{C}} \simeq \Delta_{X}^{*}\left(\underline{\mathbb{V}}^{\mathscr{H}} \otimes \underline{\mathscr{L}}\right)\left[-d_{X}\right]=:\left(\underline{\mathbb{V}}^{\mathscr{H}} \otimes \underline{\mathscr{C l}}\right)\left[-d_{X}\right]
$$

where $\otimes$ denotes the tensor product in $\mathrm{D}^{\mathrm{b}}(\operatorname{MHM}(X, Q))$ as defined in [19] and [18].

\section{3 - Comparison and further reduction}

5.3.1 - Proposition. The functor

$$
\operatorname{real}_{X}: \mathrm{D}^{\mathrm{b}}(\operatorname{MHM}(X, \mathrm{Q})) \rightarrow \mathrm{D}_{\mathscr{C}}^{\mathrm{b}}(X, Q)_{\mathscr{D}}
$$

is compatible with the $\mathrm{VMHS}(\mathrm{X}, \mathrm{Q}) \mathrm{ad}_{\mathrm{a}}$-module structures defined in $5.2 .4-$ and 5.2.2-.

5.3.2 - Remark that $\underline{V} \circledast \mathbb{Q}_{X}^{\mathscr{H}}=\underline{V}^{\mathscr{H}}$ via the isomorphism $(6.1 .6-1)$ and $\operatorname{real}_{X}\left({\underline{V^{\mathscr{C}}}}^{\mathscr{H}}\right)=\underline{\mathrm{V}}^{\mathscr{H}}$. Hence, it follows from 5.3.1 - and 5.1.4- that, for every $\mathscr{\mathscr { C }}$ in $\mathrm{D}^{\mathrm{b}}(\mathrm{MHM}(X, Q))$, one has a commutative square

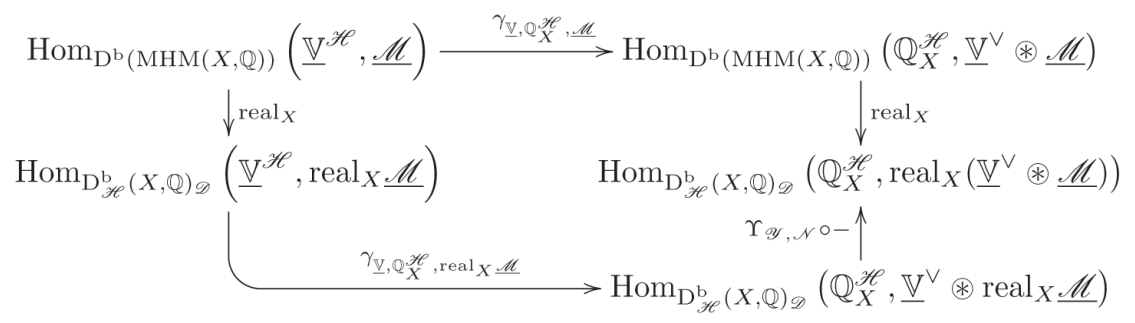

and the rigidity of the tensor category $\operatorname{VMHS}(\mathrm{X}, \mathrm{Q})_{\mathrm{ad}}$ ensures by 5.1.3 - that the horizontal arrows are isomorphisms of Q-vector spaces. We therefore reduce the proof of 1.2 .5 - to the case $\underline{N}=Q_{X}^{\mathscr{H}}$ and $\underline{\mathscr{l}} \in \mathrm{D}^{\mathrm{b}}(\mathrm{MHM}(X, Q))$.

5.3.3 - The simplest way to prove 5.3.1 - is to check that the category $\mathrm{S}_{\mathscr{H}}^{\mathrm{b}}(X, \mathrm{Q})_{\mathscr{D}}$ of stupid mixed Hodge complexes has a $\operatorname{VMHS}(\mathrm{X}, \mathrm{Q})_{\mathrm{ad}}$-module structure which is compatible with both the module structures defined on $\mathrm{C}^{\mathrm{b}}(\mathrm{MHM}(X, Q))$ and $\mathrm{D}_{\mathscr{H}}^{\mathrm{b}}(X, Q)_{\mathscr{D}}$ via the functor $\mathrm{G}$ and the functor $\mathrm{C}$.

5.3.4 - Let us first construct this module structure. Let $\mathscr{\mathscr { C }}$ and $\mathscr{L b}^{\prime}$ be two stupid mixed Hodge complexes respectively on $X$ and $Y$. One defines an 
intermediate diagrams on the product $X \times Y$ by

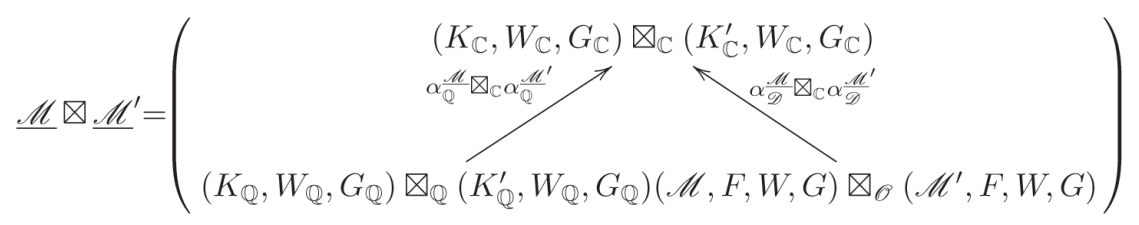

It follows from the construction that one has an isomorphism

$$
\operatorname{Gr}_{G}^{i} \operatorname{Gr}_{n}^{W}\left(\underline{\mathscr{C}} \otimes \underline{\mathscr{C}^{\prime}}\right)[i]=\bigoplus_{i_{1}+i_{2}=i} \bigoplus_{n_{1}+n_{2}=n} \operatorname{Gr}_{G}^{i_{1}} \operatorname{Gr}_{n_{1}}^{W}(\underline{\mathscr{C}})\left[i_{1}\right] \otimes \operatorname{Gr}_{G}^{i_{2}} \operatorname{Gr}_{n_{2}}^{W}\left(\underline{\mathscr{C}^{\prime}}\right)\left[i_{2}\right]
$$

in $\mathrm{D}_{\mathrm{rh}}^{\mathrm{b}} \mathrm{F}\left(\mathscr{D}_{X}, Q\right)$. Since $\mathrm{Gr}_{G}^{i_{1}} \mathrm{Gr}_{n_{1}}^{W}(\mathscr{\mathscr { C }})\left[i_{1}\right]$ is a pure Hodge module of weight $n_{1}$ on $X$ and $\operatorname{Gr}_{G}^{i_{2}} \operatorname{Gr}_{n_{2}}^{W}(\mathscr{L})\left[i_{2}\right]$ is a pure Hodge module of weight $n_{2}$ on $Y$, the algebraic analog of [19, 3.21] (see also [19, 3.28]) ensures that $\operatorname{Gr}_{G}^{i} \operatorname{Gr}_{n}^{W}\left(\mathscr{L} \otimes \mathscr{L}^{\prime}\right)[i]$ is a pure Hodge module of weight $n$. This shows that the external product of two stupid mixed Hodge complexes is also a stupid mixed Hodge complex and one gets therefore a bifunctor

$$
凶: \mathrm{S}_{\mathscr{H}}^{\mathrm{b}}(X, \mathrm{Q})_{\mathscr{D}} \times \mathrm{S}_{\mathscr{H}}^{\mathrm{b}}(Y, Q)_{\mathscr{Q}} \rightarrow \mathrm{S}_{\mathscr{H}}^{\mathrm{b}}(X \times Y, \mathrm{Q})_{\mathscr{D}} .
$$

5.3.5 - Consider now a morphism $f: X \rightarrow Y$ of smooth algebraic varieties and a stupid hodge diagram $\mathscr{\mathscr { C }}$ on $Y$. Assume that $\mathscr{\ell}$ is noncharacteristic with respect to $f$. One then defines a mixed intermediate diagram

$$
f_{\mathscr{O}}^{\sharp} \underline{\mathscr{M}}=\left(\begin{array}{cccc}
f^{-1}\left(K_{\mathbb{C}}, W_{\mathbb{C}}, G_{\mathbb{C}}\right)\{d\} & \operatorname{Sp}\left(f_{\mathscr{Q}}^{*}(\mathscr{M}, W, G)\right) \\
f^{-1} \alpha_{\mathbb{Q}}^{\mathscr{M}}\{d\} & f^{-1} \alpha_{\mathscr{\mathscr { H }}}\{d\} & f^{\mathrm{M}}\{d\} & \mathrm{Id} \\
f^{-1}\left(K_{\mathbb{Q}}, W_{\mathbb{Q}}, G_{\mathbb{Q}}\right)\{d\} & f^{-1} \operatorname{Sp}(\mathscr{M}, W, G)\{d\} f_{\mathscr{O}}^{*}(\mathscr{M}, F, W, G)
\end{array}\right)
$$

and set

$$
f_{\mathscr{O}}^{+} \underline{\mathscr{M}}:=\mathrm{Co}\left(f_{\mathscr{O}}^{\sharp} \underline{\mathscr{M l}}\right) .
$$

From the definition we have

$$
\operatorname{Gr}_{G}^{i} \operatorname{Gr}_{n}^{W}\left(f_{\mathscr{O}}^{\sharp} \underline{\mathscr{C}}\right)=f_{\mathscr{O}}^{\sharp}\left(\operatorname{Gr}_{G}^{i} \operatorname{Gr}_{n-d \underline{W}}^{W}\right)
$$

in $\mathrm{D}_{\mathrm{rh}}^{\mathrm{b}} \mathrm{F}\left(\mathscr{D}_{X}, \mathrm{Q}\right)$. We have

$$
{ }^{\mathrm{p}} \underline{\mathrm{H}}^{k}\left(\operatorname{Gr}_{G}^{i} \operatorname{Gr}_{n}^{W}\left(f_{\mathscr{O}}^{+} \underline{\mathscr{C l}}\right)\right)=f_{\mathscr{O}}^{*}\left({ }^{\mathrm{p}} \underline{\underline{H}}^{k} \operatorname{Gr}_{G}^{i} \operatorname{Gr}_{n-d}^{W} \underline{\mathscr{C l}}\right) .
$$

Since by assumption ${ }^{\mathrm{P}} \underline{H}^{k} \mathrm{Gr}_{G}^{i} \mathrm{Gr}_{n-d}^{W} \stackrel{\mathscr{C l}}{ }$ is a pure Hodge module of weight $n-d$ for $k=i$ and vanishes for $k \neq i$, it follows immediately from 3.4.3that $f_{\mathscr{O}}^{+} \mathscr{\mathscr { L }}$ is a stupid mixed Hodge complex. 
5.3.6 - Now let $\underline{V}$ be an admissible variation of mixed Hodge structures on $X$ and $\mathscr{L}$ be a stupid mixed Hodge complex on $X$. It follows from 2.3.2that the stupid mixed Hodge complex $\underline{\mathbb{V}}^{\mathscr{H}} \otimes . \mathscr{L}$ is non characteristic with respect to $\Delta_{X}$ and we may therefore define

$$
\underline{\mathrm{V}} \circledast \underline{\mathscr{C l}}:=\left(\Delta_{X}\right)_{\mathscr{O}}^{*}\left(\underline{\mathrm{V}}^{\mathscr{H}} \otimes \underline{\mathscr{M}}\right)
$$

where the pullback $\left(\Delta_{X}\right)_{\odot}^{*}$ is defined as in 6.3 -. This defines a VMHS $(\mathrm{X}, \mathrm{Q})_{\mathrm{ad}^{-}}$ module structure on $\mathrm{S}_{\mathscr{H}}^{b}(X, Q)_{\mathscr{D}}$, the proof being the same as for mixed Hodge complexes. The next proposition is then a direct consequences of the definitions and implies 5.3.1-.

5.3.7 - Proposition. The functors

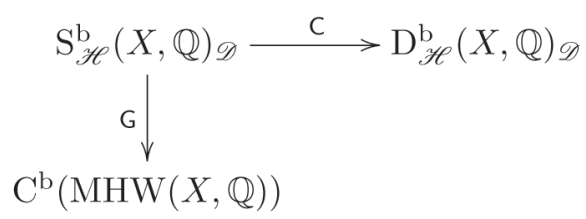

are compatible with the $\operatorname{VMHS}(\mathrm{X}, \mathrm{Q})_{\mathrm{ad}}$-module structures defined in 5.2.4-, 5.3.6 - and 5.2.2-.

\section{4 - Adjunction formula}

5.4.1 - As preliminary to the construction of an adjunction for projections between the inverse image functor and the direct image functor for mixed Q-Hodge $\mathscr{D}$-complexes, let us consider the following situation:

$$
i:=\Delta_{X} \times \operatorname{Id}_{Y}: X \times Y \hookrightarrow X \times X \times Y
$$

$\underline{V}$ is an admissible variation of mixed Hodge structures on $X$, and $\mathscr{L}$ lies in $\mathrm{D}_{\mathscr{H}}^{\mathrm{b}}(X \times Y, \mathrm{Q})_{\mathscr{O}}$. We have then a functorial morphism

$$
\underline{\mathrm{V}}^{\mathscr{H}} \otimes \underline{\mathscr{L C}} \rightarrow i_{*} i_{\mathrm{NC}}^{*}\left(\underline{\mathbb{V}}^{\mathscr{H}} \otimes \underline{\mathscr{M l}}\right)
$$

which induces on the rational component the unit

$$
\mathrm{V}_{\mathrm{Q}}\left[d_{X}\right] \otimes K_{\mathrm{Q}} \rightarrow i_{*} i^{-1}\left(\mathbb{V}_{\mathrm{Q}}\left[d_{X}\right] \otimes K_{\mathrm{Q}}\right)
$$

of the adjunction $i^{-1} \vdash i_{*}$. Here by definition $i_{\mathrm{NC}}^{*}:=i_{\mathscr{O}}^{*}\{-d\}$. Hence by definition $i_{\mathrm{NC}}^{*}$ is compatible via application of the Betti functor with the image inverse $i^{-1}$. 
5.4.2 - Let us explain the construction of this morphism. Let $\mathscr{N}$ be an object in $\mathrm{D}_{\mathscr{H}}^{\mathrm{b}}(X, Q)$ as defined in $[9,4.3 .3]$, and assume that $\mathscr{\mathscr { L }}$ is noncharacteristic. Consider the diagram:

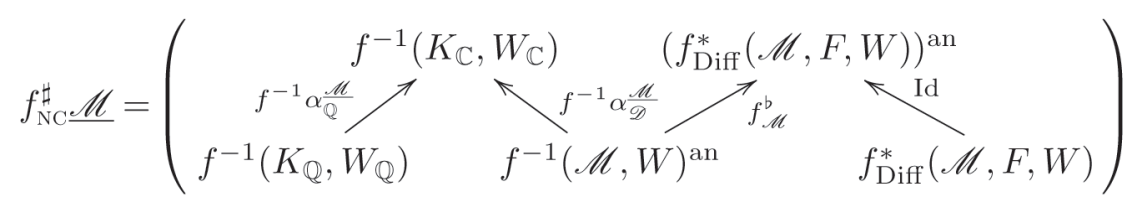

and $f_{\mathscr{O}}^{\sharp} \mathscr{L l}:=f_{\mathrm{NC}}^{\sharp} \mathscr{L l}\{d\}$. If $\mathscr{N}$ is a mixed Hodge $\mathscr{D}$-complex, then $f_{\mathscr{O}}^{\sharp} \mathrm{Sp}(\underline{\mathcal{N}})$ is isomorphic, functorially in. $\mathscr{\mathcal { N }}$, to $\operatorname{Sp}\left(f_{\mathscr{O}}^{\sharp} \cdot \mathscr{N}\right)$. Assume moreover that $f$ is proper. By definition of the pushforward, we have

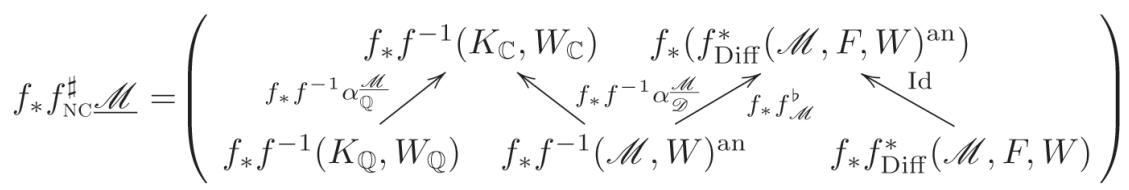

and we have therefore a morphism $\mathscr{\mathscr { C }} \rightarrow f_{*} f_{\mathrm{NC}}^{\sharp} \mathscr{\mathscr { C l }}$ which is functorial in $\mathscr{\mathscr { C }}$, which provides the desired morphism via the equivalence in $[9,4.3 .3]$.

5.4.3 - Consider a projection $p: X \times Y \rightarrow Y$ where $X, Y$ are smooth algebraic varieties and $X$ is proper. Let us check that the functor

$$
\begin{aligned}
p^{*}: \mathrm{D}_{\mathscr{H}}^{\mathrm{b}}(Y, \mathrm{Q})_{\mathscr{D}} & \rightarrow \mathrm{D}_{\mathscr{H}}^{\mathrm{b}}(X \times Y, \mathrm{Q})_{\mathscr{Q}} \\
\underline{\mathscr{L C}} & \mapsto \mathrm{Q}_{X}^{\mathscr{H}} \otimes \underline{\mathscr{M l}}
\end{aligned}
$$

is left adjoint to the direct image functor

$$
p_{*}: \mathrm{D}_{\mathscr{H}}^{b}(X \times Y, Q)_{\mathscr{D}} \rightarrow \mathrm{D}_{\mathscr{H}}^{b}(Y, Q)_{\mathscr{D}} .
$$

5.4.4 - We denote by

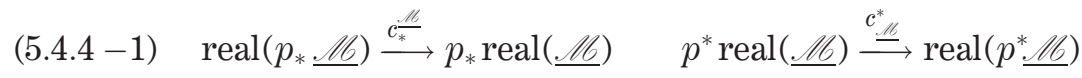

the comparison isomorphisms. Let $p, q$ be two proper morphisms we have an isomorphism

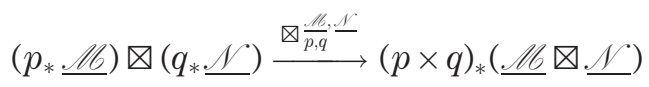

and an isomorphism

$$
c_{\underline{\mathscr{L}}, \underline{\mathcal{N}}}^{\otimes}: \operatorname{real}(\underline{\mathscr{L C}} \otimes \underline{\mathscr{N}}) \rightarrow \operatorname{real}(\underline{\mathscr{C l}}) \otimes \operatorname{real}(\underline{\mathscr{N}})
$$


5.4.5 - REMARK. The isomorphisms (5.4.4-1), (5.4.4-2) and (5.4.4-3) are compatible, more precisely the diagram

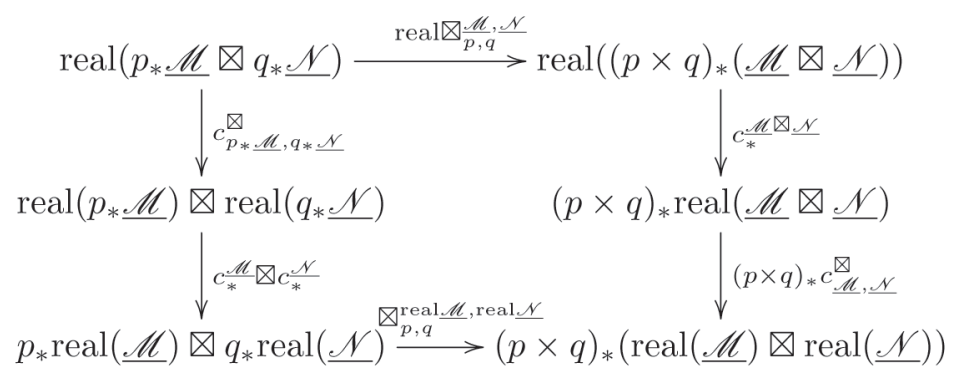

is commutative. Moreover $c_{\underline{\mathscr{C}}}^{*}=\left(c_{\mathrm{Q}_{X}^{\mathscr{K}}, \underline{\ell 6}}^{\otimes}\right)^{-1}$ for the projection $p$.

5.4.6 - Denote by $\pi$ the structural morphism of $X$. We have a natural morphism

$$
\text { 1: } \mathrm{Q}^{\mathscr{H}} \rightarrow \pi_{*} \mathrm{Q}_{X}^{\mathscr{H}}
$$

in $\mathrm{D}^{\mathrm{b}}\left(\mathrm{MHS}_{\mathrm{Q}}^{p}\right)[19,4.4 .2]$ which corresponds to the unit in the (polarizable) absolute Hodge cohomology ring $\mathrm{H}_{\mathscr{H}}^{*} \mathrm{p}(X, \mathrm{Q}(*))$ constructed in $[1, \S 5]$. Applying the realization functor to this morphism, we get a morphism

$$
\eta: \mathrm{Q}^{\mathscr{H}} \stackrel{\text { real }(\mathbf{1})}{\longrightarrow} \operatorname{real}\left(\pi_{*} \mathrm{Q}_{X}^{\mathscr{H}}\right) \stackrel{c_{*}^{Q \not \mathscr{K}}}{\longrightarrow} \pi_{*} \operatorname{real}\left(\mathrm{Q}_{X}^{\mathscr{H}}\right)=\pi_{*} \mathrm{Q}_{X}^{\mathscr{H}}
$$

in $\mathrm{D}_{\mathscr{H}}^{\mathrm{b}}(\operatorname{Spec}(\mathbb{C}), Q)_{\mathscr{V}}$. This provides a morphism

$$
a_{\underline{16}}: \underline{\mathscr{L l}} \rightarrow p_{*} p^{*} \underline{\mathscr{L l}}
$$

given by

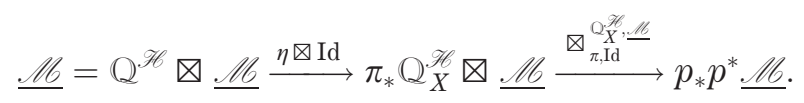

5.4.7 - Lemma. Let $\underline{\mathscr{N}} \in \mathrm{D}_{\mathscr{H}}^{\mathrm{b}}(X \times Y, \mathrm{Q})_{\mathscr{O}}$. We have a commutative diagram

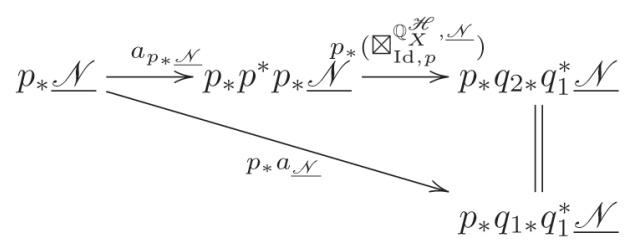


Proof. It follows immediately from the definition and the properties of the isomorphism $(5.4 .4-2)$ that one has a commutative diagram:

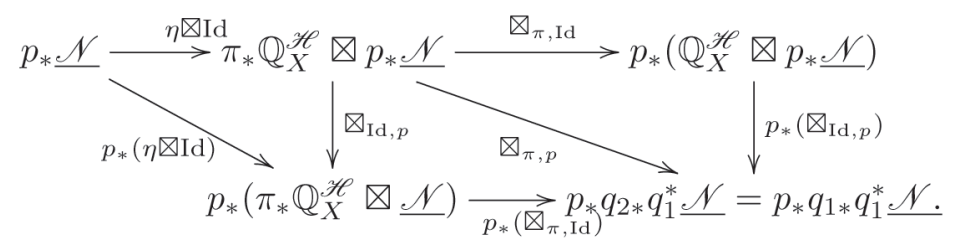

5.4.8 - Lemma. Let 16 be in $\mathrm{D}^{\mathrm{b}}(\operatorname{MHM}(Y, \mathrm{Q}))$. The square

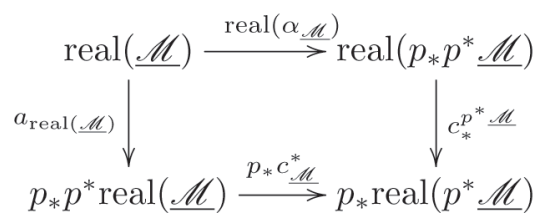

is commutative.

Proof. Using the definition and 5.4.5 - we get the following commutative diagram

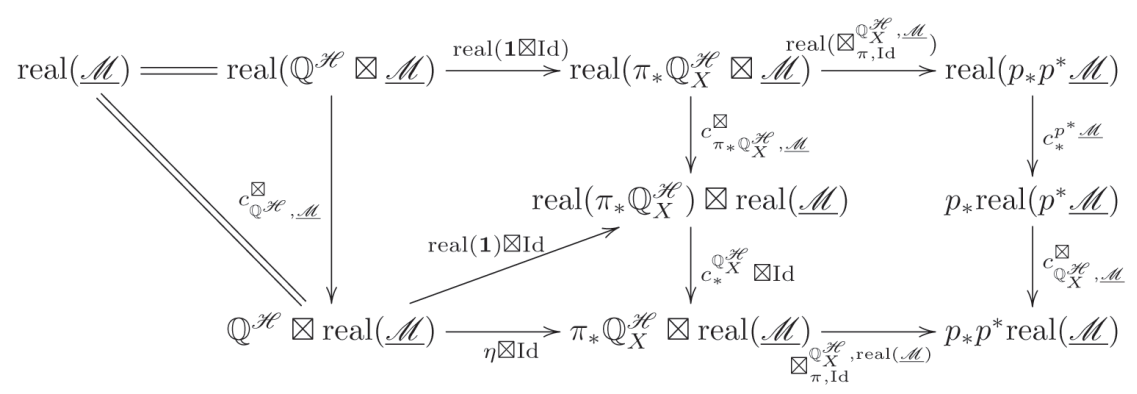

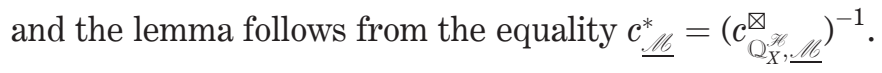

5.4.9 - Remark. Let $\sigma: Y \hookrightarrow X \times Y$ be a section of the projection $p$. Then for any $\underline{N} \in \mathrm{D}_{\mathscr{H}}^{b}(Y, Q)_{\mathscr{Q}}$ the composition

$$
\underline{\mathscr{N}} \stackrel{a_{\underline{\underline{K}}}}{\longrightarrow} p_{*} p^{*} \underline{\underline{N}} \rightarrow p_{*} \sigma_{*} \sigma_{\mathrm{NC}}^{*} p^{*} \underline{\mathscr{N}}=\sigma_{\mathrm{NC}}^{*} p^{*} \underline{\mathbb{N}}
$$


is an isomorphism in $\mathrm{D}_{\mathscr{H}}^{\mathrm{b}}(Y, Q)_{\mathscr{Q}}$ since its image under rat is an isomorphism in $\mathrm{D}_{\mathrm{c}}^{\mathrm{b}}(Y, \mathrm{Q})$.

5.4.10 - Consider the commutative diagram where $i=\Delta_{X} \times \operatorname{Id}_{Y}$

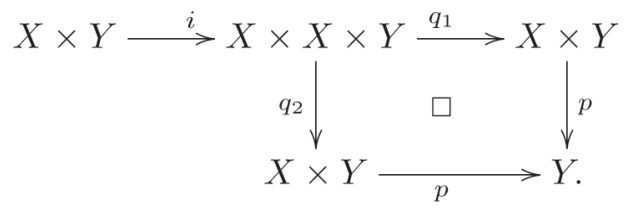

Then, given $\underline{\mathscr{N}} \in \mathrm{D}_{\mathscr{H}}^{\mathrm{b}}(Y, Q)_{\mathscr{Q}}$, we have a morphism $b_{\underline{N}}: p^{*} p_{*} \cdot \underline{\mathcal{N}} \rightarrow \underline{\mathscr{N}}$ defined by

$$
p^{*} p_{*} \underline{\mathscr{N}} \stackrel{\otimes_{\mathrm{Id}, p}}{\longrightarrow} q_{2 *} q_{1}^{*} \underline{\mathscr{N}} \rightarrow q_{2 *} i_{*} i_{\mathrm{NC}}^{*} q_{1}^{*} \underline{\mathscr{N}}=i_{\mathrm{NC}}^{*} q_{1}^{*} \underline{\mathscr{N}} \stackrel{\sim}{\rightarrow} \underline{\mathscr{N}}
$$

where the first isomorphism is given by $(5.4 .4-2)$ and the last by the inverse of the isomorphism in 5.4.9-.

5.4.11 - Lemma. Let. $\underline{\mathscr{C}}$ in $\mathrm{D}_{\mathscr{H}}^{\mathrm{b}}(Y, \mathrm{Q})_{\mathscr{D}}$ and. $\underline{\mathbb{N}}$ in $\mathrm{D}_{\mathscr{H}}^{\mathrm{b}}(X \times Y, \mathrm{Q})_{\mathscr{D}}$ The morphisms

$$
b_{p^{*} \underline{\underline{K}}} \circ p^{*} a_{\underline{\mathscr{C}}} \quad p_{*} b_{\underline{\underline{1}}} \circ a_{p_{*} \underline{\underline{H}}}
$$

are respectively the identity of $p^{*} . \mathscr{L}$ and $p_{*} \cdot \underline{N}$.

Proof. The equality $p_{*} b_{\mathcal{N}} \circ a_{p_{*} \mathscr{N}}=\operatorname{Id}$ follows from the definition and 5.4.7 - which ensure that we have a commutative diagram

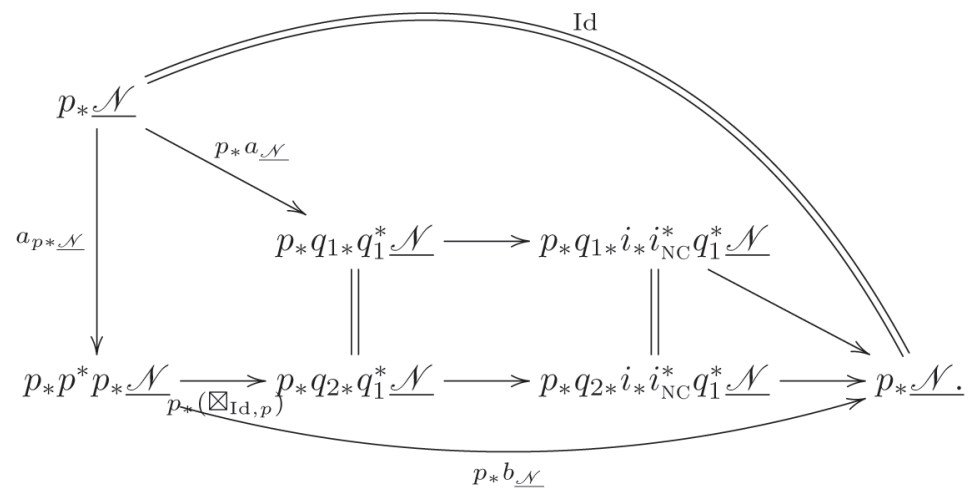

Then one has a commutative diagram 


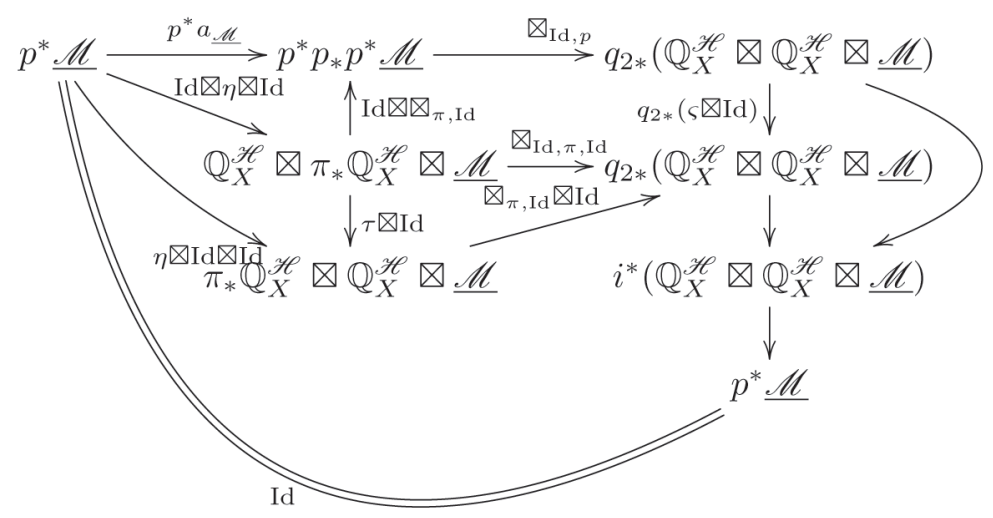

where $\tau$ and $\varsigma$ are the isomorphisms that permute the factors.

If follows from this that we have an isomorphism

$$
\operatorname{adj}: \operatorname{Hom}_{\mathrm{D}_{\mathscr{H}}^{\mathrm{b}}(X \times Y, Q)_{\mathscr{Q}}}\left(p^{*} \underline{\mathscr{L}}, \underline{\mathscr{N}}\right) \rightarrow \operatorname{Hom}_{\mathrm{D}_{\mathscr{H}}^{\mathrm{b}}(Y, \mathrm{Q})_{\mathscr{Q}}}\left(\underline{\mathscr{L}}, p_{*} \cdot \underline{\mathscr{N}}\right)
$$

which maps an element $\theta$ in the left hand side to $\operatorname{adj}(\theta)=p_{*} \theta \circ a_{\underline{\mu l}}$.

\section{5 - End of the proof}

\subsection{1 - Lemma. The square}

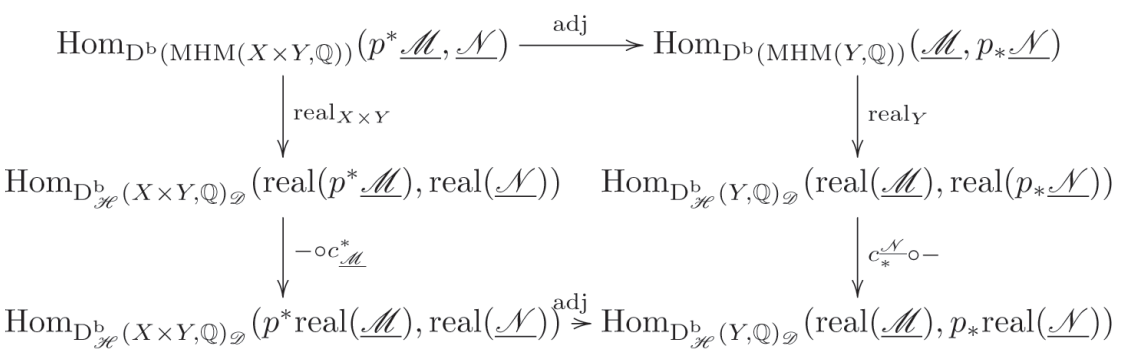

is commutative.

Proof. This follows immediately from 5.4.8-, which implies that the diagram 


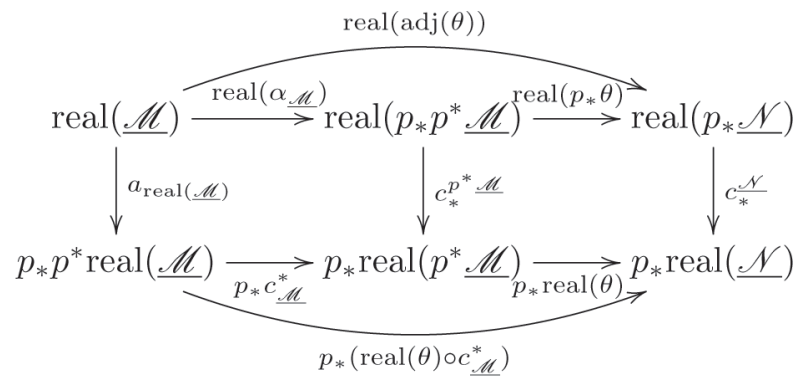

is commutative in $\mathrm{D}_{\mathscr{G}}^{b}(Y, Q)_{\mathscr{D}}$ for any morphism $\theta \in \operatorname{Hom}\left(p^{*}, \mathscr{l l}, \underline{\mathscr{N}}\right)$ in the bounded derived category of mixed Hodge modules. One of the morphism is $\operatorname{real}(\operatorname{adj}(\theta))$ while the other is $\operatorname{adj}\left(\operatorname{real}(\theta) \circ c_{\underline{\prime l}}^{*}\right)$.

Proof OF 1.2.5 - . As proved by A. Beilinson in [1], the functor real Spec (C) $_{\text {(C) }}$ is fullyfaithful. The theorem is thus a consequence of 5.5.1- and 5.3.2applied to $Y=\operatorname{Spec}(\mathrm{C})$ with $\mathscr{C}=\mathrm{Q}^{\mathscr{H}}$ the trivial mixed Hodge structure.

\section{Basic operations on mixed Q-Hodge $\mathscr{D}$-complexes}

In this section we define the two basic operations on mixed Q-Hodge complexes and prove their properties. Verification are simple but tedious. There are two main difficulties: pullbacks of diagrams necessitate to play the accordion with them and one has to check that these two operations do yield mixed Hodge complexes (this is where one need for example the behaviour of weights in the noncharacteristic case see 6.1.3-).

\section{1 - Noncharacteristic pullback I : general construction}

6.1.1 - Let $f: X \rightarrow Y$ be a morphism of smooth complex algebraic varieties and

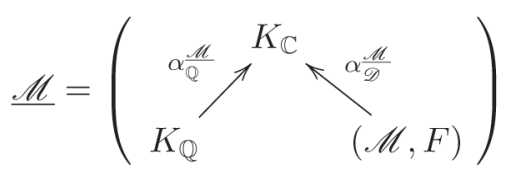

be an object in $\mathrm{D}_{\mathrm{rh}}^{\mathrm{b}} \mathrm{F}\left(\mathscr{D}_{Y}, Q\right)$. We will say that $\mathscr{C}$ is noncharacteristic with respect to $f$, or that the pullback $f_{\mathscr{O}}^{*} \mathscr{\mathscr { L }}$ is defined, when the following two conditions are satisfied: 
- ${ }^{\mathrm{P}} \underline{\mathrm{H}}^{i}(\mathscr{\mathscr { C }})$ is noncharacteristic with respect to $f$ for any integer $i \in Z$;

- $\operatorname{Gr}_{k}^{F} \mathscr{C}^{i}$ is $\mathscr{O}_{X}$-tor independent for any integer $k, i \in Z$ i.e. satisfies (3.1.1-).

6.1.2 - If these conditions are satisfied, we then define an extended diagram of length one:

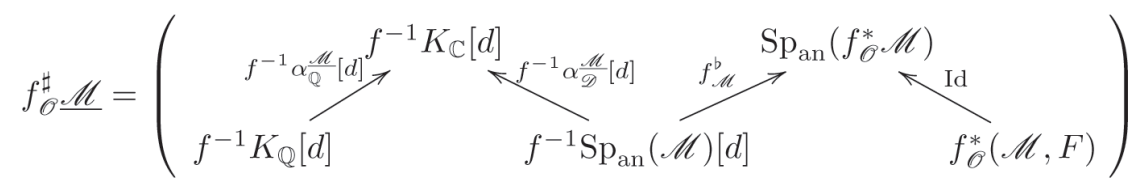

where $f_{\mathscr{L}}^{b}$ is the morphism $(3.3 .7-1)$. We know by 3.3.5 - that this morphism is a quasi-isomorphism, hence the four comparison maps are quasi-isomorphisms of complexes of sheaves of C-vector spaces on $X^{\text {an }}$, and we therefore get a pure diagram $f_{\mathscr{O}}^{\sharp} \mathscr{L}$ of length one. We then define the inverse image by

$$
f_{\mathscr{O}}^{+} \underline{\mathscr{M l}}:=\mathrm{Co}\left(f_{\mathscr{O}}^{\sharp} \underline{\mathscr{M l}}\right) .
$$

6.1.3 - Proposition. If $\mathscr{\mathscr { C }} \in \mathrm{D}_{\mathscr{H}}^{\mathrm{b}}(Y, \mathrm{Q}, n)_{\mathscr{D}}$ is a pure Hodge $\mathscr{D}$-complex of weight $n$ such that $f_{\mathscr{O}}^{*} \mathscr{L l}_{\mathrm{b}}$ is defined, then

$$
f_{\mathscr{O}}^{+} \underline{\mathscr{L}} \in \mathrm{D}_{\mathscr{H}}^{\mathrm{b}}(X, \mathrm{Q}, n+d)
$$

is a pure Hodge $\mathscr{D}$-complex of weight $n+d$.

Proof. Since $\mathscr{C}$ is a pure Hodge $\mathscr{D}$-complex of weight $n$, there exists an isomorphism in $\mathrm{D}_{\mathrm{rh}}^{\mathrm{b}} \mathrm{F}\left(\mathscr{D}_{Y}, \mathrm{Q}\right)$

$$
\underline{\mathscr{L}} \stackrel{\phi}{\rightarrow} \bigoplus_{i \in \mathrm{Z}}^{\mathrm{p}} \underline{\mathrm{H}}^{i}(\underline{\mathscr{C l}})[-i]
$$

and ${ }^{\mathrm{P}} \underline{\mathrm{H}}^{i}(\mathscr{C O})$ lies in $\mathrm{MH}(Y, \mathrm{Q}, n+i)$. By definition these perverse cohomology groups are all noncharacteristic with respect to $f$, hence we get an isomorphism

$$
f_{\mathscr{O}}^{*} \stackrel{\mathscr{C}}{\stackrel{f^{*} \phi}{\longrightarrow}} \bigoplus_{i \in Z} f_{\mathscr{O}}^{* \mathrm{p}} \underline{\underline{H}}^{i}(\underline{\mathscr{C}})[-i] .
$$

It follows from 3.4.3 - that $f_{\mathscr{O}}^{* \mathrm{P}} \underline{\mathrm{H}}^{i}(\underline{\mathscr{C l}})$ is a Hodge module of weight 
$n+i+d$. Given an integer $k \in Z$, we have therefore

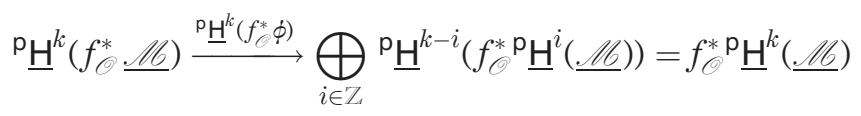

which is a Hodge module of weight $n+d+k$. Now $(6.1 .3-1)$ and $(6.1 .3-2)$ imply that $f_{\mathscr{O}}^{*} \mathscr{\mathscr { L }}$ satisfies the decomposability condition and the weight $n+d$ condition. Hence $f_{\mathscr{O}}^{*} \mathscr{\mathscr { L }}$ is a pure Hodge $\mathscr{D}$-complex of weight $n+d$.

6.1.4 - Consider now a mixed diagram $\mathscr{M}$. We will say that $\mathscr{M}$ is noncharacteristic with respect to $f$ or that $f_{\mathscr{O}}^{*} \mathscr{L}$ is defined if the $\mathrm{Gr}_{n}^{W} \mathscr{L}$ 's are noncharacteristic in the sense of $6.1 .1-$. One then defines an extended mixed diagram of length one

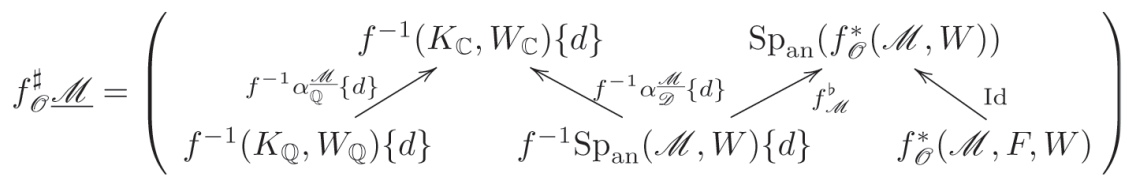

and defines the inverse image by

$$
f_{\mathscr{O}}^{+} \underline{\mathscr{L}}:=\operatorname{Co}\left(f_{\mathscr{O}}^{\sharp} \underline{\mathscr{C l}}\right) .
$$

We can define similarly a pullback for noncharacteristic mixed Hodge $\mathscr{D}$ complexes of higher length. We have a canonical isomorphism

$$
\operatorname{Gr}_{n}^{W}\left(f_{\mathscr{O}}^{\sharp} \underline{\mathscr{C}}\right)=f_{\mathscr{O}}^{\sharp}\left(\mathrm{Gr}_{n-d}^{W} \underline{\mathscr{C}}\right)
$$

If $\mathscr{L}$ is a mixed Hodge $\mathscr{D}$-complex, then by assumption $\mathrm{Gr}_{n-d}^{W} \mathscr{\mathscr { L }}$ is a pure Hodge $\mathscr{D}$-complex of weight $n-d$. It follows from 6.1.3 - that $(6.1 .4-1)$ is a pure Hodge $\mathscr{D}$-complex of weight $n$ and therefore $f_{\mathscr{O}}^{+} \mathscr{\mathscr { C }}$ is a mixed Hodge $\mathscr{D}$-complex.

6.1.5 - REMARK. The pullback functor $f_{\odot}^{+}$is not well defined on the triangulated category $\mathrm{D}_{\mathscr{H}}^{b}(Y, Q)_{\mathscr{Q}}$. More precisely if $\underline{\mathscr{C}}$ and $\mathscr{N}$ are noncharacteristic mixed Hodge complexes and $\underline{u}: \underline{\mathscr{L}} \rightarrow \underline{\mathscr{N}}$ is a morphism in $\mathrm{K}_{\mathscr{H}}^{\mathrm{b}}(Y, \mathrm{Q})_{\mathscr{Q}}$, then the morphism $f_{\mathscr{O}}^{+} \underline{u}$ is well defined whereas it is not defined a priori if $\underline{u}$ is only a morphism in $\mathrm{D}_{\mathscr{H}}^{\mathrm{b}}(Y, Q)_{\mathscr{D}}$. Indeed a mixed Hodge complex Betti quasi-isomorphic to a noncharacteristic mixed Hodge complex is not necessarily noncharacteristic. However we will use in the sequel the following remark: if $\underline{u}$ is obtained via a chain of 
morphisms in $\mathrm{K}_{\mathscr{H}}^{\mathrm{b}}(Y, \mathrm{Q})_{\mathscr{D}}$

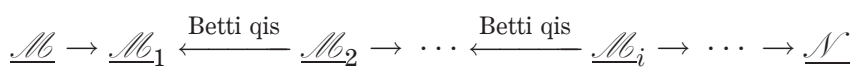

in which all the mixed Hodge complexes involved are noncharacteristic then $f_{\mathscr{O}}^{+} \underline{u}$ is a well defined morphism in $\mathrm{D}_{\mathscr{H}}^{b}(X, Q)_{\mathscr{Q}}$.

6.1.6 - Let $\underline{V}$ be an admissible variation of mixed Hodge structure on $Y$. We denote by $f^{*} \underline{V}$ the pullback of $\underline{V}$ along $f$ which is an admissible variation of mixed Hodge structures on $X$. By definition we have

$$
\operatorname{Ex}\left[{ }^{\mathscr{H}}\left(f^{*} \underline{\mathbb{V}}\right)\right]:=\left(\begin{array}{ccc}
\operatorname{DR}_{\text {an }}\left(f_{\mathscr{O}}^{*}(\mathscr{V}, W)\right) & \operatorname{DR}_{\text {an }}\left(f_{\mathscr{Q}}^{*}(\mathscr{V}, W)\right) \\
\alpha^{-1}\left(\mathbb{V}_{\mathbb{Q}}, W_{\mathbb{Q}}\right) & \operatorname{DR}_{\text {an }}\left(f_{\mathscr{O}}^{*}(\mathscr{V}, W)\right) & (\mathscr{V}, F, W)
\end{array}\right)
$$

while

$$
f_{\mathscr{O}}^{\sharp}\left(\mathscr{H}^{\underline{V}}\right):=\left(\begin{array}{ccc}
f^{-1} \mathrm{DR}_{\text {an }}(\mathscr{V}, W) & \operatorname{DR}_{\text {an }}\left(f_{\mathscr{V}}^{*}(\mathscr{V}, W)\right) \\
f^{-1} \alpha_{\mathbb{Q}}^{\mathbb{V}} & f_{\mathscr{V}}^{\text {b }} & (\mathscr{V}, F, W) \\
f^{-1}\left(\mathbb{V}_{\mathbb{Q}}, W_{\mathbb{Q}}\right) & f^{-1} \mathrm{DR}_{\text {an }}(\mathscr{V}, W) & (\mathscr{V})
\end{array}\right) .
$$

Since $f_{\mathscr{V}}^{b} \circ f^{-1} \alpha \frac{\mathrm{Y}}{Q}=\alpha_{Q}^{f^{*} \underline{V}}$, we have thus a canonical isomorphism of left mixed Hodge complexes on $X$ given by the naive morphism of left Hodge complexes:

$$
\beta(f, \underline{\mathrm{V}})=\left(\mathrm{Id}, f_{\mathscr{V}}^{b}, f_{\mathscr{V}}^{b}, \mathrm{Id}, \mathrm{Id}\right): f_{\mathscr{O}}^{\sharp}\left({ }^{\mathscr{H}} \underline{\mathrm{V}}\right) \rightarrow \operatorname{Ex}\left[{ }^{\mathscr{H}}\left(f^{*} \underline{\mathrm{V}}\right)\right] .
$$

Passing to (right) mixed Hodge complexes we get an isomorphism

$$
f_{\mathscr{O}}^{\sharp}\left(\underline{\mathrm{V}}^{\mathscr{H}}\right) \rightarrow \operatorname{Ex}\left[\left(f^{*} \underline{\mathrm{V}}\right)^{\mathscr{H}}\right]
$$

which we still denote by $\beta(f, \underline{V})$. By adjunction we get an isomorphism

$$
\varrho(f, \underline{\mathbb{V}}): f_{\mathscr{O}}^{+}\left(\underline{\mathbb{V}}^{\mathscr{H}}\right) \rightarrow\left(f^{*} \underline{\mathrm{V}}\right)^{\mathscr{H}}
$$

of mixed Hodge complexes.

\section{2 - Noncharacteristic pullback II : 2-functoriality}

6.2.1 - Remark that functoriality is too stringent a condition to hold for mixed Q-Hodge $\mathscr{D}$ - complexes. However a weak functoriality holds and we will now check that the pullback constructed in $6.1-$ is 2 -functorial with 
respect to morphisms of varieties. In other work we will construct a comparison isomorphism in $\mathrm{D}_{\mathscr{H}}^{\mathrm{b}}(W, \mathrm{Q})_{\mathscr{Q}}$

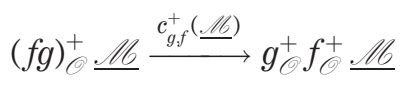

for any sequence $W \stackrel{g}{\rightarrow} X \stackrel{f}{\rightarrow} Y$ of morphisms of smooth algebraic varieties and any mixed Hodge complex $\mathscr{\mathscr { C }}$ such that $\mathscr{M}$ is noncharacteristic with respect to $f$ and $f^{+} \mathscr{M b}$ is noncharacteristic with respect to $g$, and then check that these comparison isomorphisms satisfy the required coherency properties:

6.2.2 - Proposition. Let $W \stackrel{g}{\rightarrow} X \stackrel{f}{\rightarrow} Y \stackrel{h}{\rightarrow} Z$ be a sequence of morphisms of smooth algebraic varieties. The square

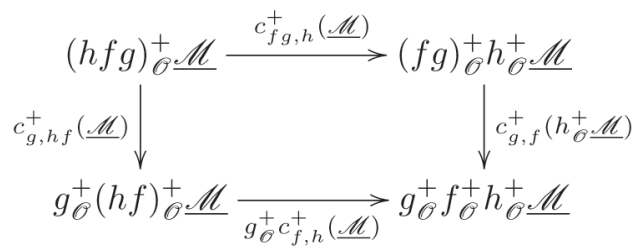

is commutative for any mixed Hodge complex $\mathscr{L}$ such that $\mathscr{l}, h_{\odot}^{+} \mathscr{L}$, $f_{\mathscr{O}}^{+} h_{\mathscr{O}}^{+} \underline{\mathscr{L}}$ and $g_{\mathscr{O}}^{+} f_{\mathscr{O}}^{+} h_{\mathscr{O}}^{+} \underline{\mathscr{L l}}$ are defined.

The rest of the section is devoted to these easy though tedious verifications.

6.2.3 - Let $r \geqslant 0$ be an integer. As preliminaries to the construction of $(6.2 .1-1)$ let us remark that we have at our disposal two isomorphisms respectively in $\mathrm{D}_{\mathscr{C}}^{\mathrm{b}}(X, \mathrm{Q})_{\mathscr{O}}^{r+1}$ and $\mathrm{D}_{\mathscr{C}}^{\mathrm{b}}(X, \mathrm{Q})_{\mathscr{O}}^{r+2}$

$$
\begin{aligned}
& \varepsilon(f, \underline{\mathscr{L C}}): f_{\mathscr{O}}^{\sharp} \underline{\mathscr{L l}} \rightarrow \operatorname{Ex}\left[f_{\mathscr{O}}^{+} \underline{\mathscr{M l}}\right] \\
& \gamma(f, \underline{\mathscr{L C}}): f_{\mathscr{O}}^{\sharp} \operatorname{Ex}[\underline{\mathscr{L C}}] \rightarrow \operatorname{Ex}\left[f_{\mathscr{O}}^{\sharp} \underline{\mathscr{L C}}\right]
\end{aligned}
$$

for any given mixed Hodge complex $\mathscr{L}$ of length $r$ on $Y$ which is non characteristic with respect to $f$. The first isomorphism is provided unit of the adjunction Co $\vdash$ Ex. To write down the second one it is simpler to work with left mixed Hodge complexes. To go back to (right) mixed Hodge complexes one simply uses the side changing functors. We will assume $r=0$ otherwise we would have to write diagrams which wouldn't 
fit into the page. The construction is nonetheless the same for bigger $r$. By definition

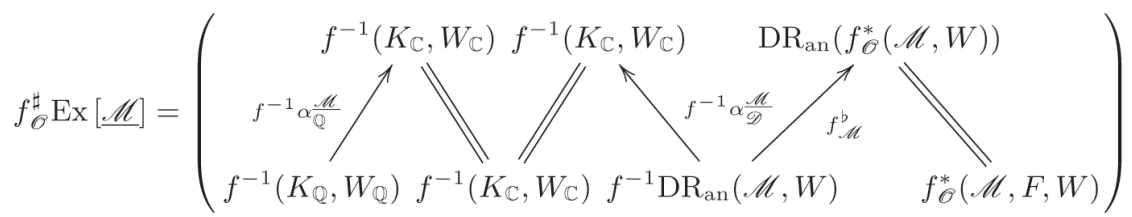

while

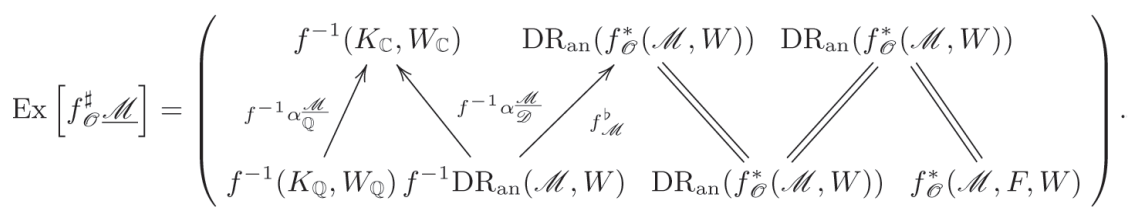

Hence we have two naive morphisms of left mixed Hodge complexes of length 2

$$
f_{\mathscr{O}}^{\sharp} \operatorname{Ex}[\underline{\mathscr{L C}}] \stackrel{u(f, \underline{\mathscr{M C}})}{\longleftarrow} \Gamma(f, \underline{\mathscr{L C}}) \stackrel{v(f, \underline{\mathscr{M C}})}{\longleftarrow} \operatorname{Ex}\left[f_{\mathscr{O}}^{\sharp} \underline{\mathscr{L C}}\right]
$$

given by

$$
\begin{aligned}
& u(f, \underline{\mathscr{C l}})=\left(\mathrm{Id}, \mathrm{Id}, f^{-1} \alpha_{\mathscr{D}}, f^{-1} \alpha \frac{\mathscr{C l}}{\mathscr{D}}, \mathrm{Id}, \mathrm{Id}, \mathrm{Id}\right) \\
& v(f, \underline{\mathscr{C b}})=\left(\mathrm{Id}, \mathrm{Id}, \mathrm{Id}, f_{\mathscr{L}}^{b}, f_{\mathscr{L}}^{b}, \mathrm{Id}, \mathrm{Id}\right)
\end{aligned}
$$

where $\Gamma(f, \underline{\mathscr{C}})$ is by definition the left mixed Hodge complex of length 2

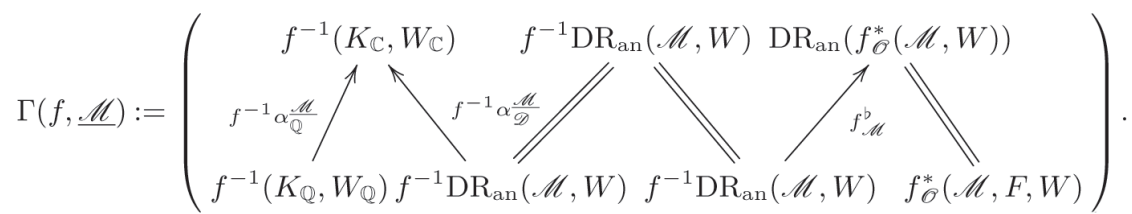

The morphism $\gamma(f, \underline{\mathscr{C}})$ is defined by $\gamma(f, \underline{\mathscr{C l}}):=v(f, \underline{\mathscr{C l}}) u(f, \underline{\mathscr{C l}})^{-1}$. In the sequel we will use the isomorphisms $(6.2 .3-1)$ and $(6.2 .3-2)$ only for mixed Hodge complexes of length $\leqslant 1$.

6.2 .4 - Let us fix a sequence $W \stackrel{g}{\rightarrow} X \stackrel{f}{\rightarrow} Y$ of morphisms of smooth algebraic varieties and a mixed Hodge complex $\mathscr{l}$ of length $r$ such that $\underline{\mathscr{C}}$ and $f_{\mathscr{O}}^{+} \mathscr{\mathscr { C }}$ are noncharacteristic. Since the contraction functor doesn't change the $\mathscr{D}$-component the last condition simply means that $f_{\mathscr{O}}^{\sharp} \mathscr{\mathscr { C }}$ is noncharacteristic. To get $(6.2 .1-1)$, it is enough to construct an isomorph- 
ism in $\mathrm{D}_{\mathscr{H}}^{b}(W, Q)_{\mathscr{O}}^{r+2}$

$$
\operatorname{Ex}\left[(f g)_{\mathscr{O}}^{\sharp} \underline{\mathscr{L}}\right] \stackrel{c_{g, f}^{\sharp}(\mathscr{\mathscr { C }})}{\longrightarrow} g_{\mathscr{O}}^{\sharp} f_{\mathscr{O}}^{\sharp} \underline{\mathscr{L l}} .
$$

Indeed (6.2.1-1) will then be obtained from (6.2.4-1) as follows: the functor Ex being an equivalence, the composition

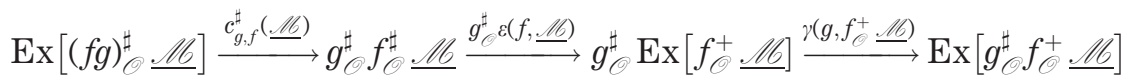
defines an isomorphism in $\mathrm{D}_{\mathscr{H}}^{\mathrm{b}}(W, Q)_{\mathscr{Q}}^{r+1}$

$$
(f g)_{\odot}^{\sharp} \underline{\mathscr{L}} \rightarrow g_{\odot}^{\sharp} f_{\odot}^{+} \underline{\mathscr{L l}}
$$

and $(6.2 .1-1)$ is the image of this isomorphism by the contraction functor Co. Hence it remains to define (6.2.4-1). Here again we assume $r=0$ for size reasons and work with left mixed Hodge complexes. Let us denote by $s$ the composition $f g$. By definition

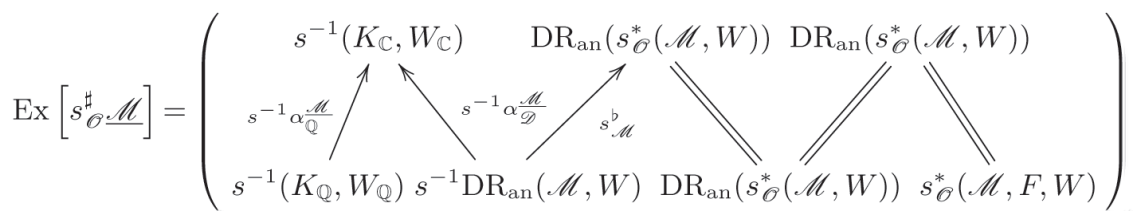

while

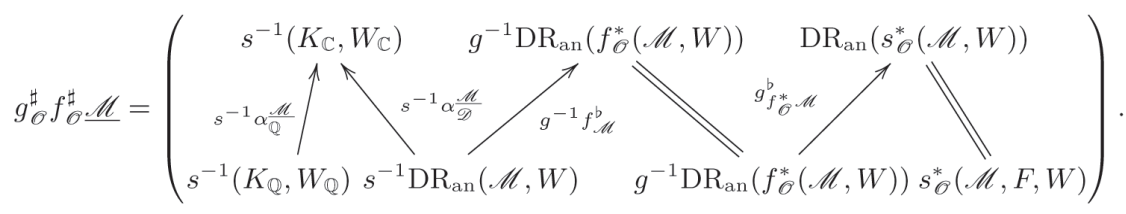

The isomorphism (6.2.4-1) is then defined as the inverse of the naive morphism of mixed Hodge complexes of length 2

$$
\left(\mathrm{Id}, \mathrm{Id}, \mathrm{Id}, g_{f_{\mathscr{O}}^{*}}^{b} \mathscr{\mathscr { C }}, g_{f_{\mathscr{O}}^{*} \mathscr{M}}^{b}, \mathrm{Id}, \mathrm{Id}\right): g_{\mathscr{O}}^{\sharp} f_{\mathscr{O}}^{\sharp} \underline{\mathscr{C l}} \rightarrow \operatorname{Ex}\left[s_{\mathscr{O}}^{\sharp} \underline{\mathscr{C l}}\right] .
$$

6.2.5 - REMARK. By functoriality of the unit morphism, the square

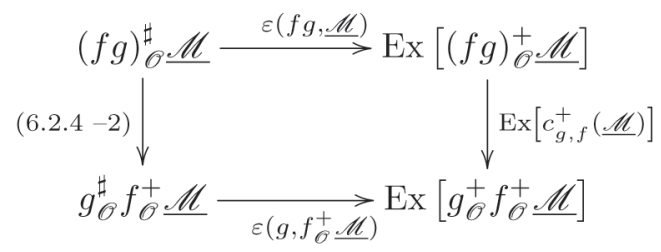


is commutative. Unfolding the definition, it follows that the diagram $(6.2 .5-1)$

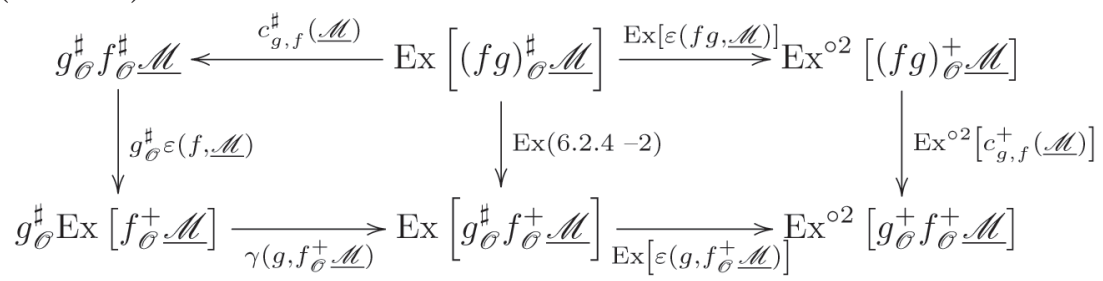

is also commutative.

The next lemma is easily checked:

6.2.6 - Lemma. Let $\mathcal{N}$ be a mixed Hodge complex of length $r$ such that $\underline{\mathbb{N}}$ and $f_{\mathscr{O}}^{+} \underline{\mathcal{N}}$ are non characteristic. Then the square

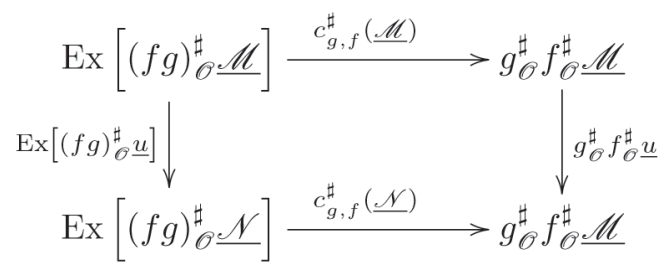

is commutative for any morphism $\underline{u}: \underline{\mathscr{C}} \rightarrow \underline{\mathcal{N}}$ in $\mathrm{K}_{\mathscr{H}}^{\mathrm{b}}(Y, Q)_{\mathscr{D}}$.

If one takes the time to write down explicitly the mixed Hodge complexes involved, the next lemma is an obvious consequence of 3.3.7-:

6.2.7 - Lemma. The diagram

$$
\begin{aligned}
& \operatorname{Ex}\left[(f g)_{\mathscr{O}}^{\sharp} \operatorname{Ex} \underline{\mathscr{M}}\right] \longrightarrow \operatorname{Ex}^{\circ 2}\left[(f g)_{\mathscr{O}}^{\sharp} \underline{\mathscr{M}}\right]
\end{aligned}
$$

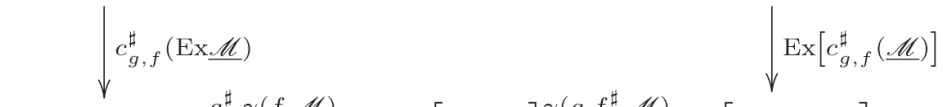

$$
\begin{aligned}
& g_{\mathscr{O}}^{\sharp} f_{\mathscr{O}}^{\sharp} \operatorname{Ex} \underline{\mathscr{M}} \stackrel{g_{\mathscr{O}}^{\sharp} \gamma(f, \underline{\mathscr{M}})}{\longrightarrow} g_{\mathscr{O}}^{\sharp} \operatorname{Ex}\left[f_{\mathscr{O}}^{\sharp} \underline{\mathscr{M}}\right] \stackrel{\gamma\left(g, f_{\mathscr{O}}^{\sharp}, \mathscr{M}\right)}{\longrightarrow} \operatorname{Ex}\left[g_{\mathscr{O}}^{\sharp} f_{\mathscr{O}}^{\sharp} \underline{\mathscr{M}}\right]
\end{aligned}
$$

is commutative.

For the convenience of the reader we expand (6.2.7-1) in Figure 1 (DR stands there for the analytic de Rham complex).

6.2.8 - Let us now prove 6.2.2-. We fix a sequence of morphisms of smooth algebraic varieties

$$
W \stackrel{g}{\rightarrow} X \stackrel{f}{\rightarrow} Y \stackrel{h}{\rightarrow} Z
$$


and a mixed Hodge complex $\mathscr{K}$ satisfying the assumptions of the proposition. To keep notation short we set $s=f g, t=h f$ and $r=h f g$. Once again it suffices to write down explicitly the mixed Hodge complexes involved as done in Figure 2 to see that 3.3.7 - immediately implies:

6.2.9 - Lemma. The diagram

$(6.2 .9-1)$

$$
\begin{aligned}
& \mathrm{Ex}^{\circ 2}\left[(h f g)_{\mathscr{O}}^{\sharp} \frac{\mathscr{M}}{\operatorname{Ex}\left[c_{f g, h}^{\sharp}(\underline{M})\right]} \longrightarrow \operatorname{Ex}\left[(f g)_{\mathscr{O}}^{\sharp} h_{\mathscr{O}}^{\sharp} \frac{\mathscr{M}}{\longrightarrow}\right]\right. \\
& \operatorname{Ex}\left[c_{g, h f}^{\sharp}(\underline{\mathscr{M}})\right] \downarrow \\
& \downarrow c_{g, f}^{\sharp}\left(h_{\mathscr{O}}^{\sharp}, \mathscr{M}\right)
\end{aligned}
$$

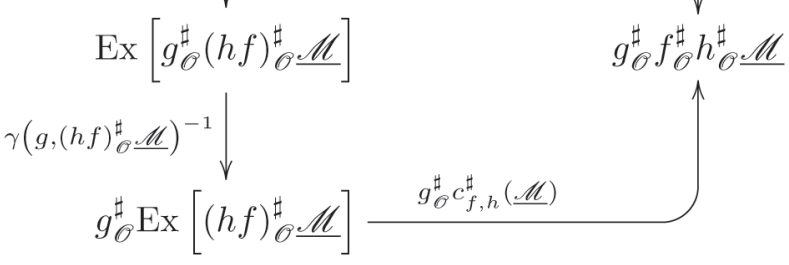

is commutative.

Proof OF 6.2.2 - . We just have to reduce the assertion to 6.2.9 - . For this remark that the results previously proved ensure that we have the commutative diagram depicted in Figure 3. Hence it follows from 6.2.9(see the subdiagram d5) that the image of the square $(6.2 .2-1)$ by the functor $\mathrm{Ex}^{\circ 3}$ is commutative. Since the extension functor $\mathrm{Ex}$ is an

\begin{tabular}{|c|c|}
\hline \begin{tabular}{|l|l|l|l|}
$\mathrm{d} 1$ & $\mathrm{~d} 4$ & $\mathrm{~d} 6$ & $\mathrm{~d} 12$ \\
\end{tabular} & Commutativity of $(6.2 .5-1)$ \\
\hline \begin{tabular}{|l|l|l|l|}
$\mathrm{d} 7$ & $\mathrm{~d} 8$ & $\mathrm{~d} 9$ & $\mathrm{~d} 10$ \\
\cline { 1 - 3 } & $\mathrm{d} 10$ \\
\end{tabular} & Functoriality in $\underline{\mathscr{L}}$ of $\gamma(f, \underline{\mathscr{L}})$ \\
\hline d11 & Functoriality in $\underline{\mathscr{L}}$ of $\varepsilon(f, \underline{\mathscr{C}})$ \\
\hline $\mathrm{d} 3$ & $6.2 .7-$ \\
\hline $\mathrm{d} 2$ & $6.2 .6-$ \\
\hline
\end{tabular}
equivalence, the proposition follows. We list below the results used to ensure the commutativity of the diagram in Figure 3:

6.3 - Noncharacteristic pullback III : a cheap trick

6.3.1 - Let $f: X \rightarrow Y$ be an isomorphism and $\mathscr{L}$ be a mixed Hodge complex on $Y$. Since the map $f_{\text {, b }}^{b}$ is then a filtered isomorphism we may consider instead of the mixed Hodge complex of length one 
defined in 6.1.4-, the mixed Hodge complex

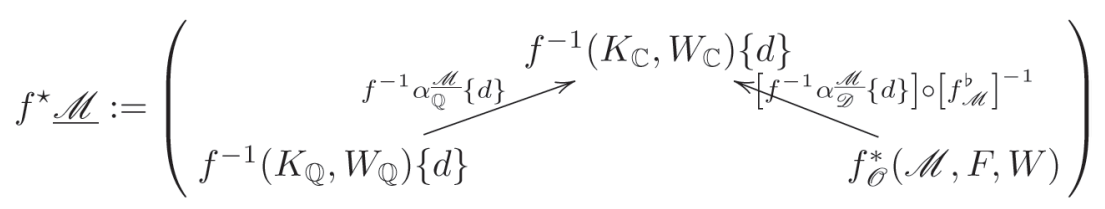

or equivalently if we have a left mixed Hodge complex to begin with :

$$
f^{\star} \underline{\mathscr{M}}:=\left(\begin{array}{c}
f_{f_{\mathscr{O}}^{*}}^{*}(\mathscr{M}, F, W) \\
f^{-1}\left(K_{\mathbb{Q}}, W_{\mathbb{Q}}\right)
\end{array}\right) .
$$

The naive morphism $\theta(f, \underline{\mathscr{C l}}):=\left(\mathrm{Id}, \mathrm{Id}, f^{-1} \alpha \frac{\mathscr{L l}}{\mathscr{D}}, f^{-1} \alpha_{\mathscr{D}} \circ\left[f_{\mathscr{L}}^{b}\right]^{-1}, \mathrm{Id}\right)$ (here we used left mixed Hodge complexes) provides an isomorphism

$$
\theta(f, \underline{\mathscr{L C}}): f_{\mathscr{O}}^{\sharp} \underline{\mathscr{L C}} \rightarrow \operatorname{Ex}\left(f_{\mathscr{O}}^{\star} \underline{\mathscr{M l}}\right)
$$

and we let

$$
\vartheta(f, \underline{\mathscr{L C}}): f_{\mathscr{O}}^{+} \underline{\mathscr{L C}} \rightarrow f_{\mathscr{O}}^{\star} \underline{\mathscr{L l}}
$$

be its adjoint i.e.:

$$
\theta(f, \underline{\mathscr{L}})=\operatorname{Ex}[\vartheta(f, \underline{\mathscr{C l}})] \circ \varepsilon(f, \underline{\mathscr{L}}) .
$$

Let us now fix a sequence of isomorphisms $W \stackrel{g}{\rightarrow} X \stackrel{f}{\rightarrow} Y$ and a mixed Hodge complex $\mathscr{L l}_{\mathfrak{b}}$ in $\mathrm{K}_{\mathscr{H}}^{\mathrm{b}}(Y, \mathrm{Q})_{\mathscr{D}}$. The next lemma is an immediate consequence of 3.3.7 - (see Figure 5 in which DR stands there for the analytic de Rham complex).

6.3.2 - Lemma. The diagram

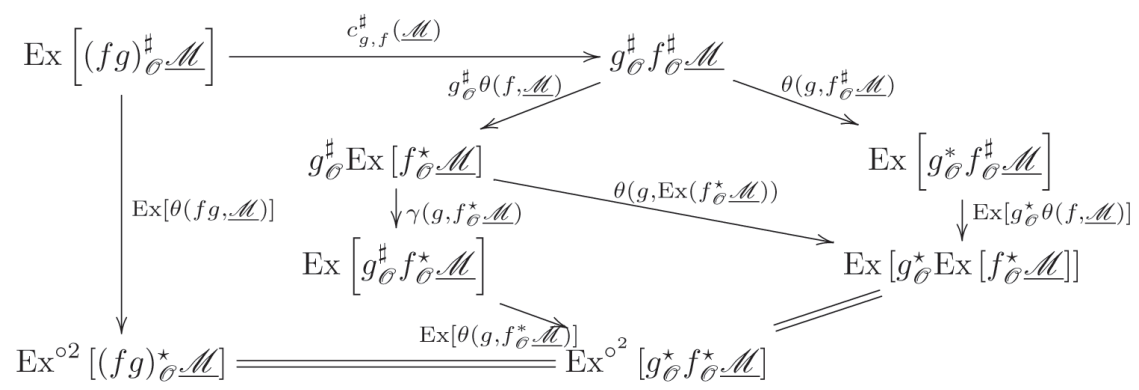

is commutative 
6.3.3 - Lemma. The diagram

$(6.3 .3-1)$

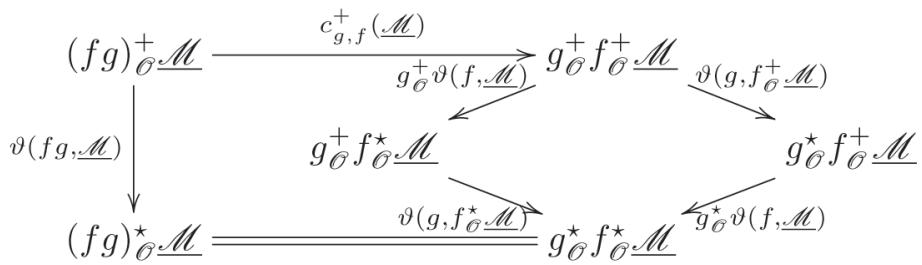

is commutative.

Proof. We check only the commutativity of the inner square of $(6.3 .3-1)$, the proof for the other square is similar. Using $(6.2 .5-1)$ and the functoriality in $\mathscr{N}$ of the morphisms $\gamma(g, \underline{N})$ and $\varepsilon(g, \underline{\mathscr{N}}), 6.3 .2-$ provides the following commutative diagram:

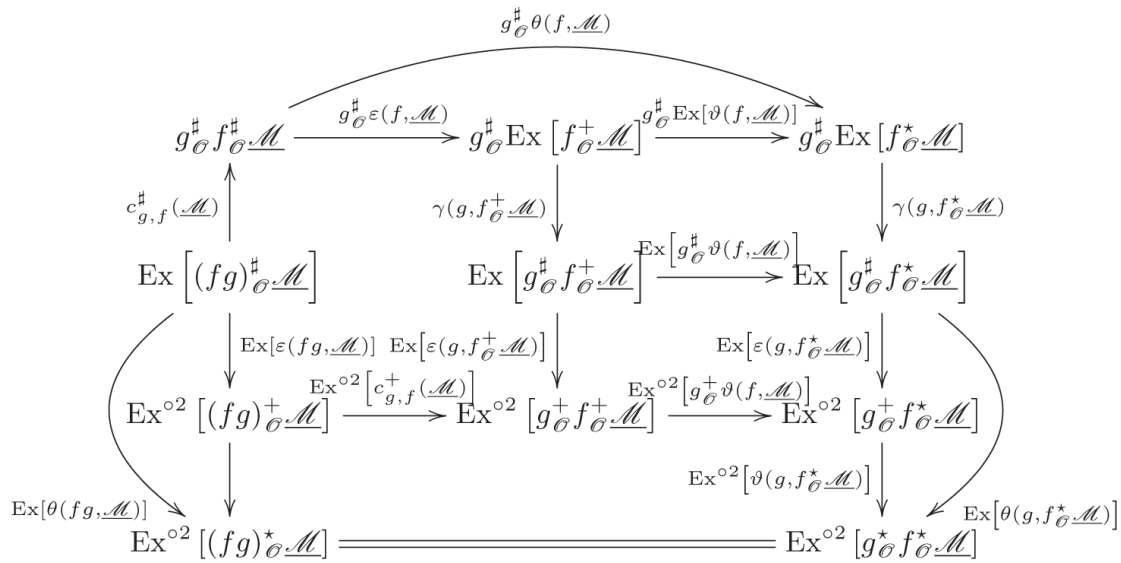

Hence the image of the square $(6.3 .3-1)$ by the functor $\mathrm{Ex}^{\circ 2}$ is a commutative. Since Ex is an equivalence, it implies the lemma.

6.3.4 - A nice feature of 2-functors is that they are lax enough to be redefined when needed. We now redefine the noncharacteristic image inverse. Let $f: X \rightarrow Y$ be a morphism of smooth algebraic varieties and a noncharacteristic mixed Hodge complex $\mathscr{\mathscr { L }}$. We finally set

$$
f_{\mathscr{O}}^{*} \underline{\mathscr{C}}:= \begin{cases}f_{\mathscr{O}}^{\star} \underline{\mathscr{C l}} & \text { if } f \text { is an isomorphism; } \\ f_{\mathscr{O}}^{+} \underline{\mathscr{C l}} & \text { otherwise. }\end{cases}
$$


Let $W \stackrel{g}{\rightarrow} X \stackrel{f}{\rightarrow} Y$ be a sequence of morphisms of smooth algebraic varieties. The definition of the comparison isomorphisms

$$
(f g)_{\mathscr{O}}^{*} \underline{\mathscr{L C}} \stackrel{c_{g f f} \stackrel{\mathscr{L})}{\longrightarrow}}{\longrightarrow} g_{\mathscr{O}}^{*} f_{\mathscr{O}}^{*} \underline{\mathscr{L C}}
$$

now depends on whether the morphisms $g, f$ and $g f$ are isomorphisms or not:

\begin{tabular}{|c|c|c|c|}
\hline \multicolumn{3}{|c|}{ CASES } & DEFINITION OF $c_{g, f}(\underline{\mathscr{C l}})$ \\
\hline Case 1 & \multicolumn{2}{|c|}{ All of them are isomorphisms. } & $c_{g, f}^{\star}(\underline{\mathscr{C l}})=\mathrm{Id}$ \\
\hline CASE 2 & \multicolumn{2}{|c|}{ None of them are isomorphisms. } & $c_{g, f}^{+} \underline{(\mathscr{C l})}$ \\
\hline \multirow{3}{*}{ CASE 3} & \multirow{3}{*}{$\begin{array}{l}\text { Only one of them is an } \\
\text { isomorphism }\end{array}$} & 3a: It's $g f$ & $c_{g, f}^{+}(\underline{\mathscr{L}}) \circ \vartheta(g f, \underline{\mathscr{L}})^{-1}$ \\
\hline & & 3b: It's $g$ & $\vartheta\left(g, f_{\mathscr{O}}^{+} \underline{\mathscr{C}}\right) \circ c_{g, f}^{+}(\underline{\mathscr{C}})$ \\
\hline & & 3c: It's $f$ & $g_{\mathscr{O}}^{+} \vartheta(f, \underline{\mathscr{C}}) \circ c_{g, f}^{+}(\underline{\mathscr{C l}})$ \\
\hline
\end{tabular}

It is easy to check that the analog of 6.2.2 - holds. One only has to check the commutativity of the analog of $(6.2 .2-1)$ in all the possible cases. For example in the case $g f, h$ and $g f h$ are isomorphisms while $g, f, f h$ are not, it follows from the definition, 6.2.2 - and 6.3.3 - that

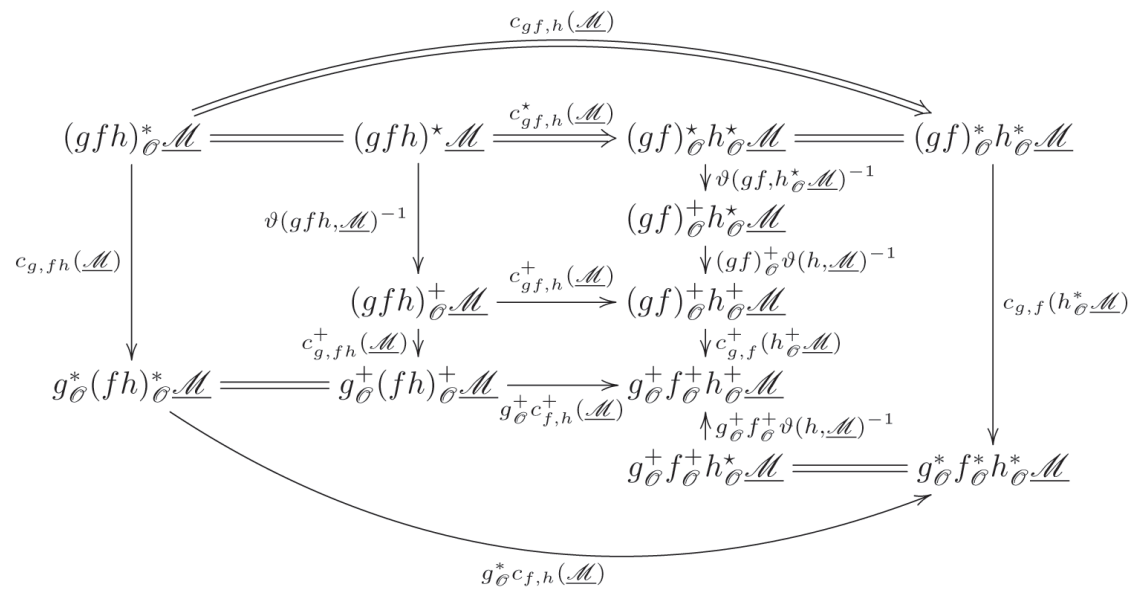

is commutative. The other cases are handled similarly. 
6.4.1 - Lemma. Let $\underline{\mathrm{V}}$ be an admissible variation of mixed Hodge structures on $Y$. Then the square

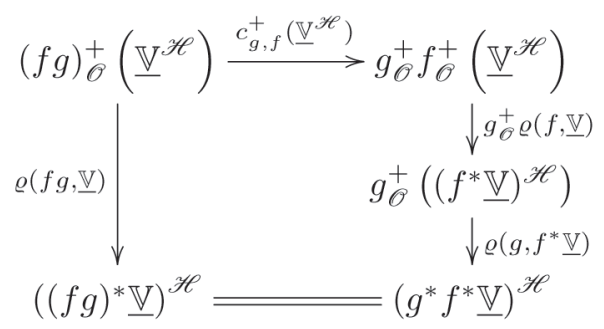

is commutative.

Proof. It is easy to check from 3.3.7 - and the definitions that the diagram

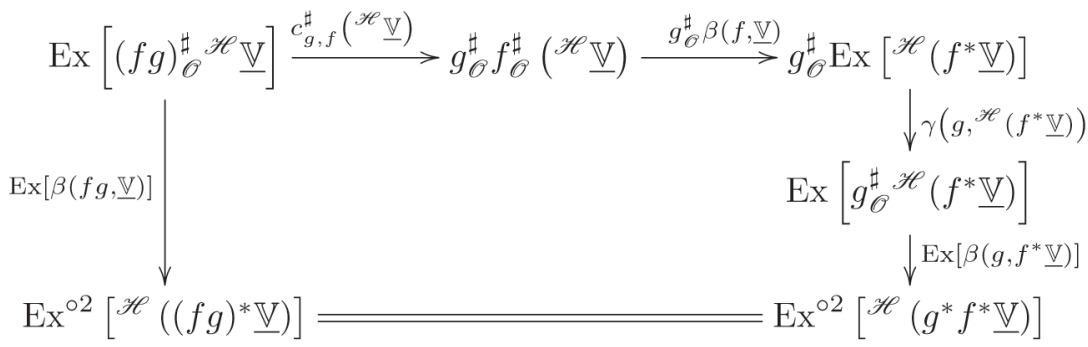

is commutative. Now consider the diagram depicted in Figure 4. By definition of the morphism $(6.1 .6-1)$ the squares $\mathrm{d} 2$ and $\mathrm{d} 6$ are commutative. The functoriality in. $\mathscr{N}$ of the morphism $\gamma(g, \mathscr{N})$ ensures the commutativity of d3. Similarly the commutativity of the square d5 follows from the functoriality in $\mathscr{N}$ of the morphism $\varepsilon(g, \mathscr{N})$. The square $\mathrm{d} 1$ is commutative by $(6.2 .5-1)$. Hence the square $\mathrm{d} 4$ is commutative, which means that the image by of the square $(6.4 .1-1)$ by the functor $\mathrm{Ex}^{\circ 2}$ is commutative. Since the extension functor Ex is an equivalence, the lemma follows.

\section{5 - External products}

6.5.1 - Let $X$ and $Y$ be either two smooth complex algebraic varieties or two complex analytic manifolds. We denote by

$$
X \stackrel{p}{\leftarrow} X \times Y \stackrel{q}{\rightarrow} Y
$$


the two projections. Let $\mathscr{C}$ be an $\mathscr{O}_{X}$-module and $\mathscr{N}$ be an $\mathscr{O}_{Y}$-modules, their external product is defined as the $\odot_{X \times Y}$-module

$$
\mathscr{C} \otimes_{\mathscr{O}} \mathscr{N}:=\mathscr{O}_{X \times Y} \otimes_{p^{-1} \mathscr{O}_{X} \otimes q^{-1} \mathscr{O}_{Y}}\left(p^{-1} \mathscr{L} \otimes_{\mathrm{C}} q^{-1} \mathscr{N}\right) .
$$

This functor is an exact functor with respect to $\mathscr{C}$ and $\mathscr{N}$. If $\mathscr{C}$ is a left $\mathscr{D}_{X}$-module and $\mathscr{N}$ is a left $\mathscr{D}_{Y}$-modules, then $\mathscr{C} \nabla_{\mathscr{O}} \mathscr{N}$ has a natural structure of left $\mathscr{D}_{X \times Y}$-module. Indeed, since $\mathscr{D}_{X \times Y}=\mathscr{D}_{X} \otimes_{\mathscr{O}} \mathscr{D}_{Y}$, one may also described $\mathscr{C} \otimes_{\mathscr{O}} \mathscr{N}$ as the tensor product

$$
\mathscr{L} \otimes_{\mathscr{O}} \mathscr{N}=\mathscr{D}_{X \times Y} \otimes_{p^{-1} \mathscr{D}_{X} \otimes q^{-1} \mathscr{D}_{Y}}\left(p^{-1} \mathscr{L} \otimes_{\mathrm{C}} q^{-1} \mathscr{N}\right) .
$$

With this structure of left $\mathscr{D}$-module, with have for the de Rham complexes, an isomorphism of complexes

$$
\operatorname{DR}(\mathscr{C l}) \otimes_{\mathrm{C}} \mathrm{DR}(\mathscr{N}) \stackrel{\sim}{\rightarrow} \operatorname{DR}(\mathscr{C l} \otimes \mathscr{N})
$$

If $\mathscr{C}$ and $\mathscr{N}$ are right $\mathscr{D}$-modules, then one may define their external product via the side changing operations, since $\omega_{X \times Y}=\omega_{X} \bigotimes_{\mathscr{O}} \omega_{Y}$ we see that one simply gets

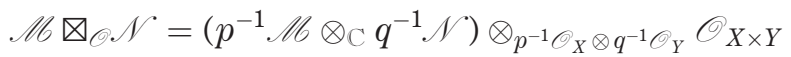

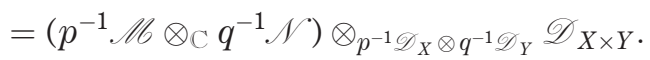

We have an isomorphism of complexes

$$
\operatorname{Sp}_{X}(\mathscr{C l}) \otimes_{\mathrm{C}} \operatorname{Sp}_{Y}(\mathscr{N}) \stackrel{\sim}{\rightarrow} \operatorname{Sp}_{X \times Y}\left(\mathscr{C l} \otimes_{\mathscr{O}} \mathscr{N}\right)
$$

which is compatible with $(6.5 .1-1)$ via the isomorphisms defined in 2.1.7 If $X$ and $Y$ are smooth algebraic complex varieties, then one has a canonical isomorphism

$$
\left(\mathscr{C} \nabla_{\mathscr{O}} \mathscr{N}\right)^{\mathrm{an}}=\mathscr{C l}^{\mathrm{an}} \nabla_{\mathscr{O}} \mathscr{N}^{\mathrm{an}} .
$$

Using the total complex associated with a bicomplex, we can extend the definition of the external product to bounded complexes of right $\mathscr{D}$-modules and one still has the isomorphisms $(6.5 .1-2)$ for bounded complexes of right $\mathscr{D}$-modules.

6.5.2 - Now assume that $X$ and $Y$ are smooth algebraic complexes varieties. If $(\mathscr{C}, F)$ and $(\mathscr{N}, F)$ are filtered right $\mathscr{D}$-modules. Then $\mathscr{C} \otimes_{\mathscr{O}} \mathscr{N}$ has a natural filtration, it is the underlying $\mathscr{D}$-module of the 
filtered $\mathscr{D}_{X \times Y}$-module

$$
\begin{aligned}
(\mathscr{C}, F) \otimes_{\mathscr{O}}(\mathscr{N}, F): & \left(p^{-1}(\mathscr{L}, F) \otimes_{\mathrm{C}} q^{-1}(\mathscr{N}, F)\right) \otimes_{p^{-1} \mathscr{O}_{X} \otimes q^{-1} \mathscr{O}_{Y}} \mathscr{O}_{X \times Y} \\
& =\left(p^{-1}(\mathscr{C}, F) \otimes_{\mathrm{C}} q^{-1}(\mathscr{N}, F)\right) \otimes_{p^{-1} \mathscr{D}_{X} \otimes^{-1} \mathscr{D}_{Y}}\left(\mathscr{D}_{X \times Y}, F\right) .
\end{aligned}
$$

Hence the filtration satisfies

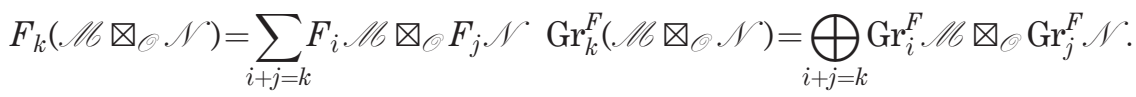

If $\mathscr{L} 6$ and $\mathscr{N}$ are coherent filtered $\mathscr{D}$-module then so is $(\mathscr{C}, F) \otimes_{\mathscr{O}}(\mathscr{N}, F)$.

6.5.3 - Let $A=\mathrm{Q}$, C. If $K \in \mathrm{D}_{\mathrm{c}}^{\mathrm{b}}(X, A)$ and $L \in \mathrm{D}_{\mathrm{c}}^{\mathrm{b}}(Y, A)$ then

$$
K \otimes_{A} L=p^{-1} K \otimes_{A} q^{-1} L
$$

lies in $\mathrm{D}_{\mathrm{c}}^{\mathrm{b}}(X \times Y, A)$ and the bifunctor $-\otimes_{A}-$ is $t$-exact for the perverse $t$-structures. One has a canonical isomorphism

$$
\left(K \otimes_{\mathrm{Q}} L\right) \otimes_{\mathrm{Q}} \mathrm{C}=\left(K \otimes_{\mathrm{Q}} \mathrm{C}\right) \otimes_{\mathrm{C}}\left(L \otimes_{\mathrm{Q}} \mathrm{C}\right)
$$

for $K \in \mathrm{D}_{\mathrm{c}}^{\mathrm{b}}(X, \mathrm{Q})$ and $L \in \mathrm{D}_{\mathrm{c}}^{\mathrm{b}}(Y, \mathrm{Q})$.

6.5.4 - Let $X$ and $Y$ be two smooth complex algebraic varieties. Let $\mathscr{L b}$ and $\mathscr{L}^{\prime}$ be two objects in $\mathrm{MF}_{\mathrm{rh}}\left(\mathscr{D}_{X}, \mathrm{Q}\right)$ and $\mathrm{MF}_{\mathrm{rh}}\left(\mathscr{D}_{Y}, Q\right)$ respectively. One defines the external product $\mathscr{1 6} \otimes \cdot \mathscr{C b}^{\prime}$ as follows:

- the underlying filtered right $\mathscr{D}_{X \times Y}$-module is $(\mathscr{L}, F) \otimes_{\mathscr{O}}\left(\mathscr{C}^{\prime}, F\right)$;

- for $A=\mathrm{Q}, \mathbb{C}$ the underlying $A$-perverse sheaf on $X^{\text {an }} \times Y^{\text {an }}$ is $K_{A} \otimes_{A} K_{A}^{\prime}$

- the comparison isomorphisms are given by

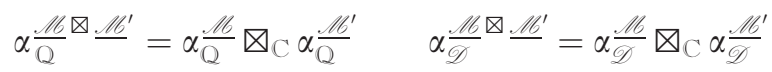

via the isomorphism (6.5.3-1) in the first case, and (6.5.1-3), (6.5.1-2) in the second case.

This provides an object. $\mathscr{L} \otimes \cdot \mathscr{C l}^{\prime}$ in $\mathrm{MF}_{\mathrm{rh}}\left(\mathscr{D}_{X \times Y}, \mathrm{Q}\right)$.

6.5.5 - Consider now two pure diagrams

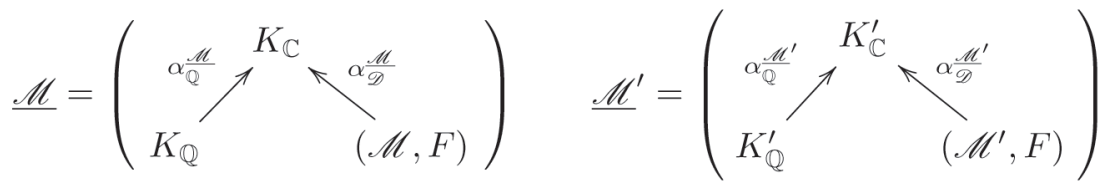


on $X$ and $Y$ respectively. Then, we have a diagram on the product $X \times Y$ :

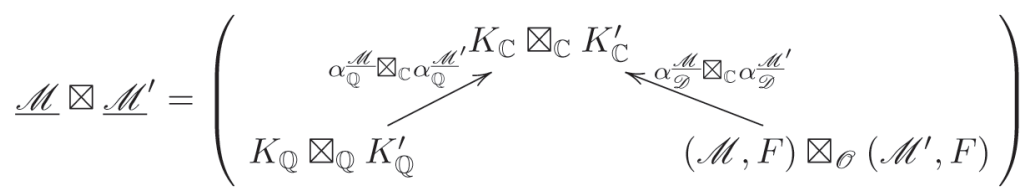

via the isomorphisms (6.5.3-1), (6.5.1-3) and (6.5.1-2).

6.5.6 - REMARK. The functor $\otimes$ is $t$-exact with respect to the perverse $t$-structure on diagram and compatible with the construction in 6.5.4-.

6.5.7 - Lemma. If $\underline{\mathscr{L}} \in \mathrm{D}_{\mathscr{H}}^{\mathrm{b}}(X, \mathrm{Q}, n)_{\mathscr{Q}}$ and $\underline{\mathcal{N}} \in \mathrm{D}_{\mathscr{H}}^{\mathrm{b}}(Y, \mathrm{Q}, m)_{\mathscr{Q}}$ are two pure Hodge $\mathscr{D}$-complexes of weight $n$ and $m$ respectively, then

$$
\underline{\mathscr{C}} \mathrm{\otimes} . \underline{\mathbb{N}} \in \mathrm{D}_{\mathscr{H}}^{\mathrm{b}}(X \times Y, \mathrm{Q}, n+m)_{\mathscr{Q}}
$$

i.e $\mathscr{L} \otimes . \mathscr{N}$ is a pure Hodge $\mathscr{D}$-complex of weight $n+m$.

Proof. By assumption there exists two isomorphism $\phi, \psi$

$$
\underline{\mathscr{C}} \stackrel{\phi}{\rightarrow} \bigoplus_{i \in Z} \underline{\mathrm{P}}^{i} \underline{\mathscr{H}}^{(\mathscr{C O})}[-i] \quad \underline{\mathscr{N}} \stackrel{\psi}{\rightarrow} \bigoplus_{j \in Z} \mathrm{P}^{\mathrm{P}} \underline{\mathrm{H}}^{j}(\underline{\mathscr{N}})[-j]
$$

and ${ }^{\mathrm{P}} \underline{\mathrm{H}}^{i}(\underline{\mathscr{C l}})$ (resp. $\left.{ }^{\mathrm{P}} \underline{\mathrm{H}}^{j}(\underline{\mathscr{N}})\right)$ is a pure Hodge modules on $X$ (resp. $Y$ ) of weight $i+n$ (resp. $j+m$ ). Hence we have an isomorphism

$$
(6.5 .7-1) \quad \underline{\mathscr{C}} \otimes \cdot \underline{\mathcal{N}} \stackrel{\phi \otimes \psi}{\longrightarrow} \bigoplus_{i, j \in \mathbb{Z}}\left({ }^{\mathrm{P}} \underline{\underline{H}}^{i}(\underline{\mathscr{C}}) \otimes^{\mathrm{P}} \underline{\mathrm{H}}^{j}(\underline{\mathscr{N}})\right)[-i-j] .
$$

By the algebraic analog of [19, 3.21] (see also [19, 3.28]), ${ }^{\mathrm{P}} \underline{\mathrm{H}}^{\mathrm{i}}(\underline{\mathscr{C}}) \otimes^{\mathrm{P}} \underline{\mathrm{H}}^{j}(\underline{\mathcal{N}})$ is a pure Hodge module of weight $n+m+i+j$ on $X \times Y$. Hence applying $\mathrm{p}^{\mathrm{H}^{k}}$ on both sides of $(6.5 .7-1)$, it follows that

$$
\underline{\mathrm{P}}^{\mathrm{P}} \underline{\mathrm{H}}^{k}(\underline{\mathscr{C l}} \otimes \cdot \underline{\mathscr{N}}) \simeq \bigoplus_{i+j=k} \underline{\mathrm{P}}^{\mathrm{H}}(\underline{\mathscr{C l}}) \otimes^{\mathrm{P}} \underline{\mathrm{H}}^{j}(\underline{\mathscr{N}})
$$

is a pure Hodge module of weight $n+m+k$. This shows that $\mathscr{L} \otimes . \mathscr{N}$ is a pure Hodge $\mathscr{D}$-complex of weight $n+m$ as desired.

6.5.8 - Now consider two mixed diagrams $\mathscr{\mathscr { L }}$ and $\mathscr{L 6}^{\prime}$ on $X$ and $Y$ as in $(4.3 .4-1)$. The external product of $\mathscr{L}$ and $\mathscr{L b}^{\prime}$ is defined as the 
mixed diagram

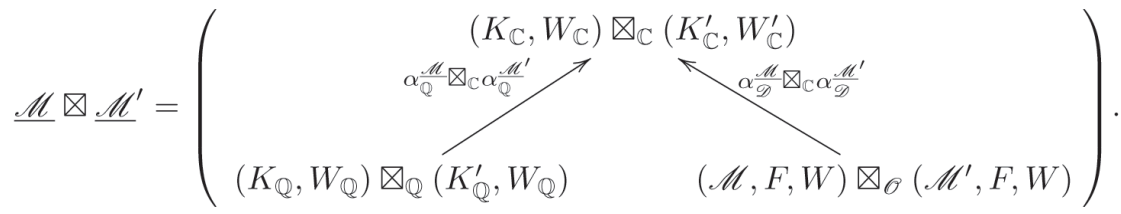

It follows from the construction that one has an isomorphism

$(6.5 .8-1)$

$$
\operatorname{Gr}_{n}^{W}\left(\underline{\mathscr{L}} \otimes \underline{\mathscr{C}^{\prime}}\right):=\bigoplus_{i+j=n} \operatorname{Gr}_{i}^{W}(\underline{\mathscr{L}}) \otimes \mathrm{Gr}_{j}^{W}\left(\underline{\mathscr{L}}{ }^{\prime}\right)
$$

of pure diagrams. The following lemma is an immediate consequence of 6.5.7 - and the isomorphism (6.5.8-1):

6.5.9 - LEMMA. If $\mathscr{\mathscr { C }} \in \mathrm{D}_{\mathscr{H}}^{\mathrm{b}}(X, \mathrm{Q})_{\mathscr{Q}}$ and $\underline{\mathscr{N}} \in \mathrm{D}_{\mathscr{H}}^{\mathrm{b}}(Y, Q)_{\mathscr{Q}}$ are two mixed Hodge $\mathscr{D}$-complexes, then

$$
\underline{\mathscr{L}} \otimes . \underline{\mathbb{N}} \in \mathrm{D}_{\mathscr{H}}^{\mathrm{b}}(X \times Y, \mathrm{Q})_{\mathscr{D}}
$$

i.e $\mathscr{L} \otimes \cdot \underline{\mathcal{N}}$ is also a mixed Hodge $\mathscr{D}$-complex.

Acknowledgments. This work was partially supported by the Humboldt foundation. The author thanks Marc Levine for an invitation to the University Duisburg-Essen where part of this work was written. 


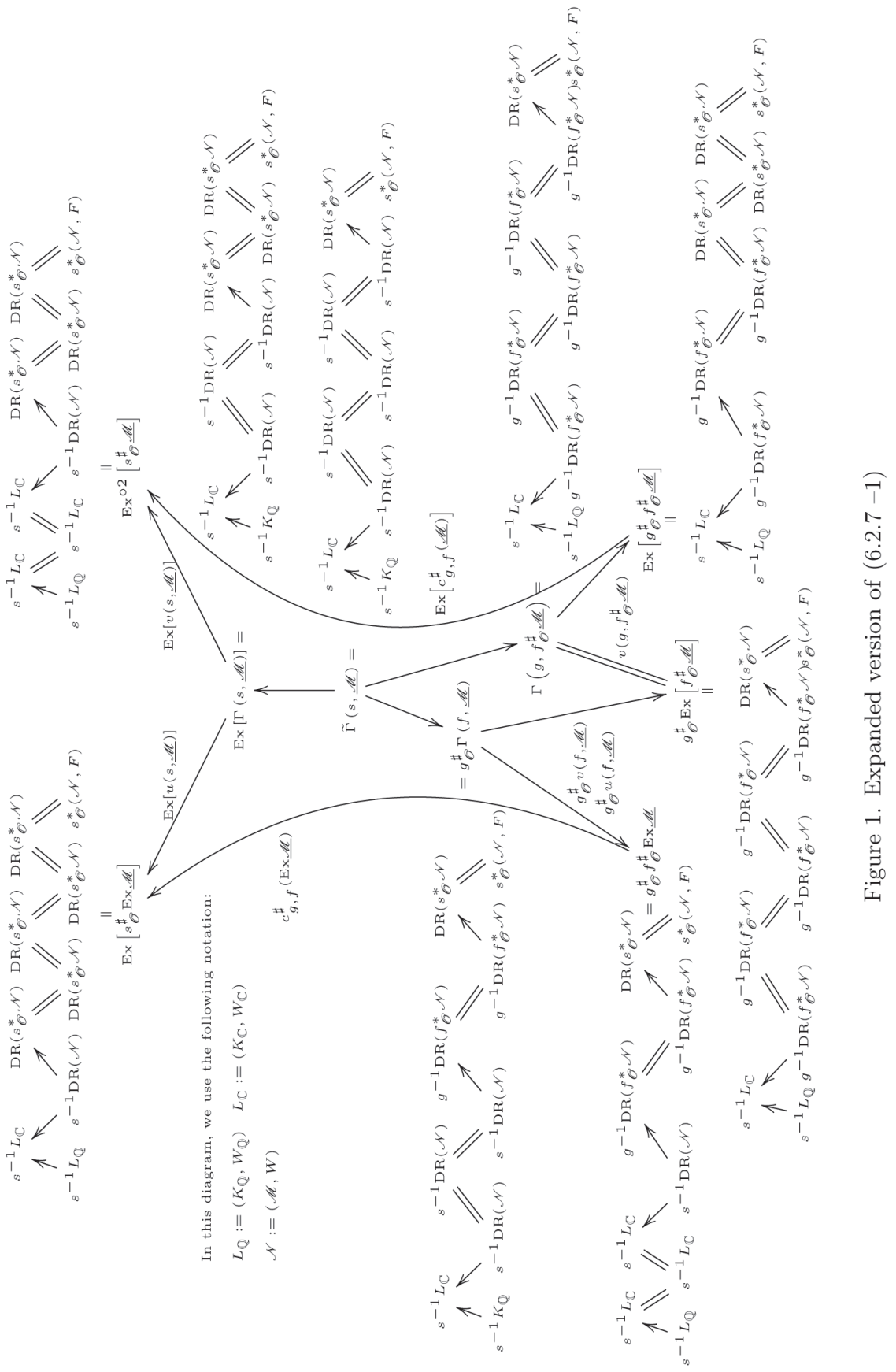




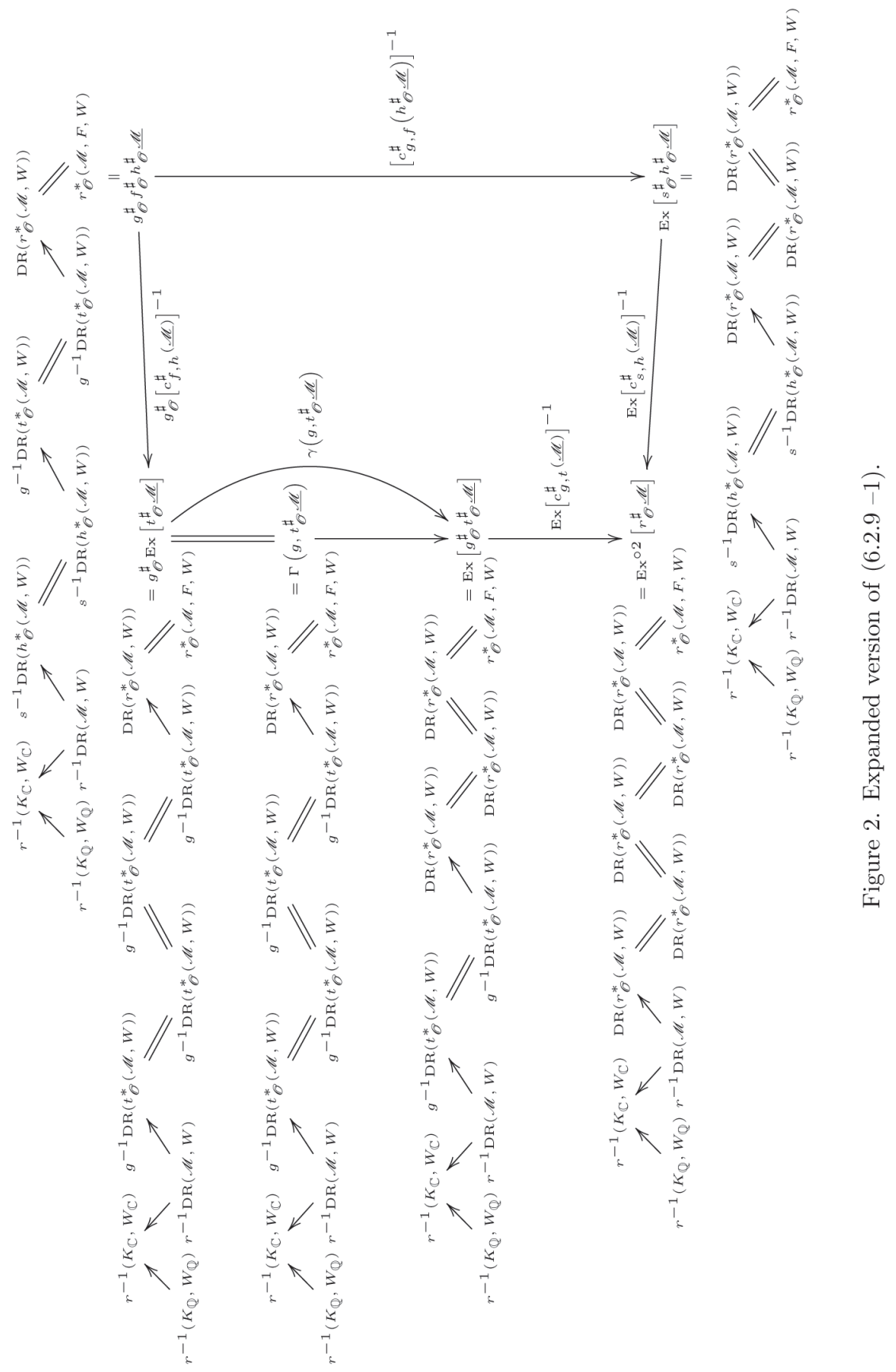


Mixed Hodge complexes and higher extensions etc.

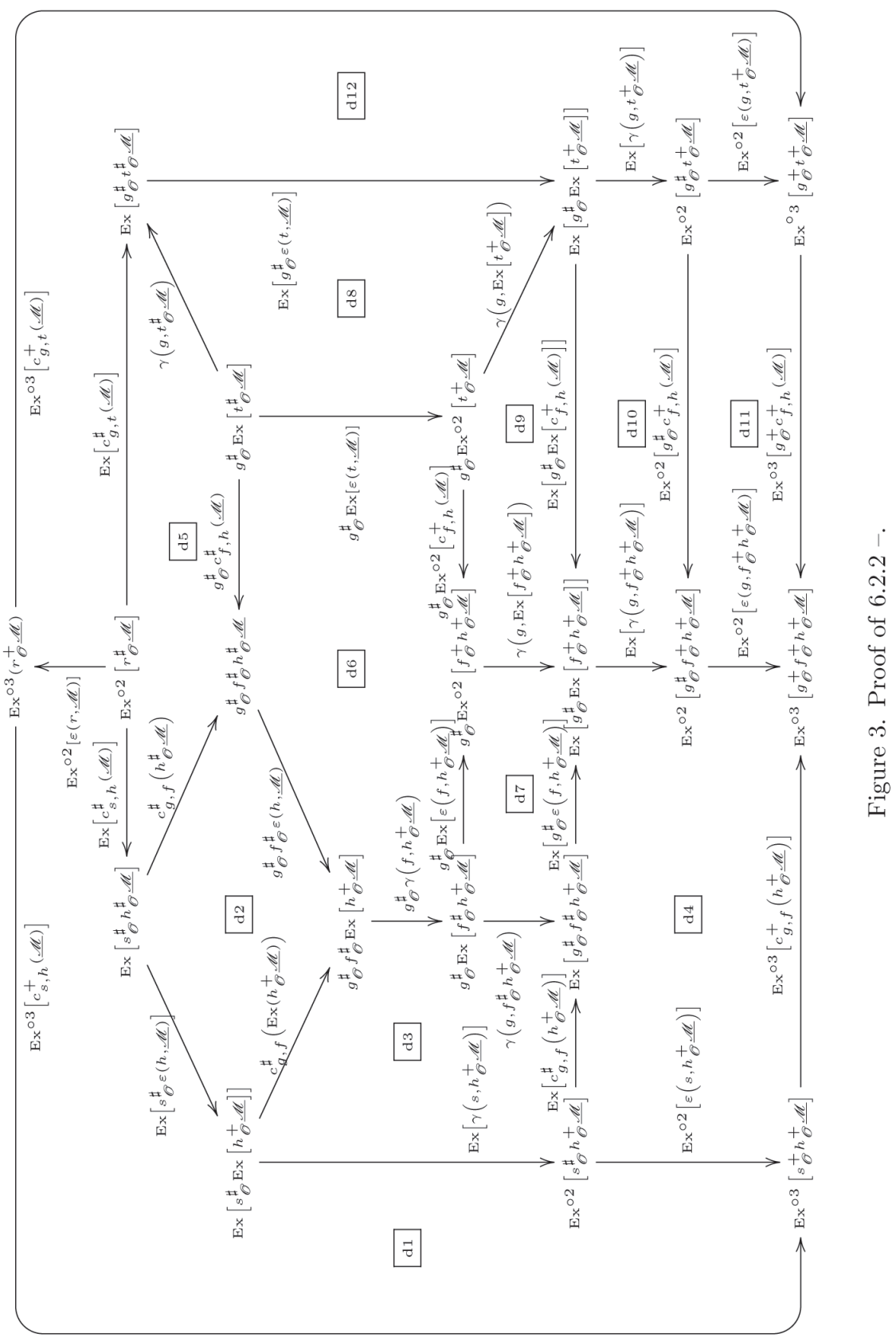




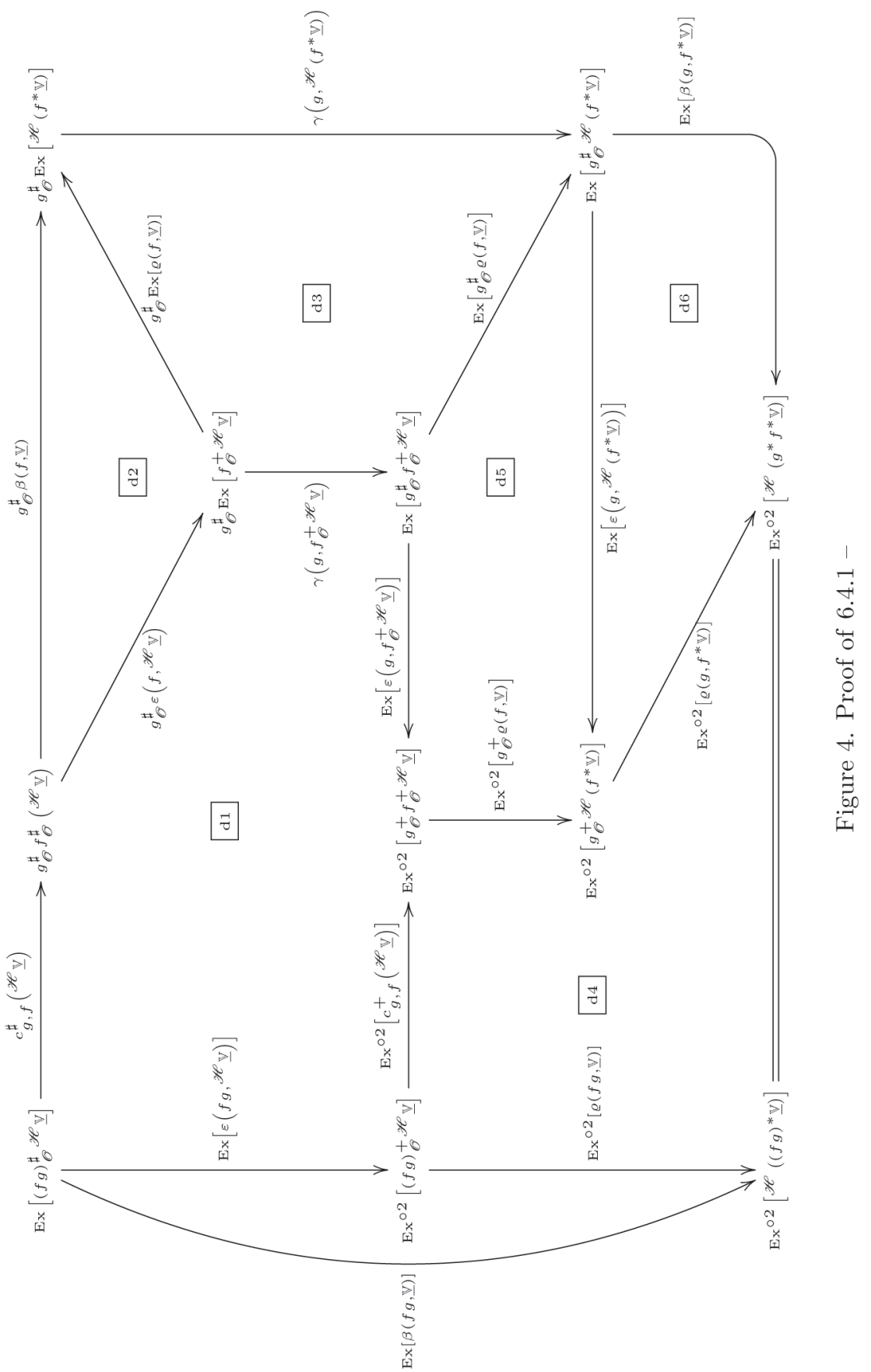


Mixed Hodge complexes and higher extensions etc.

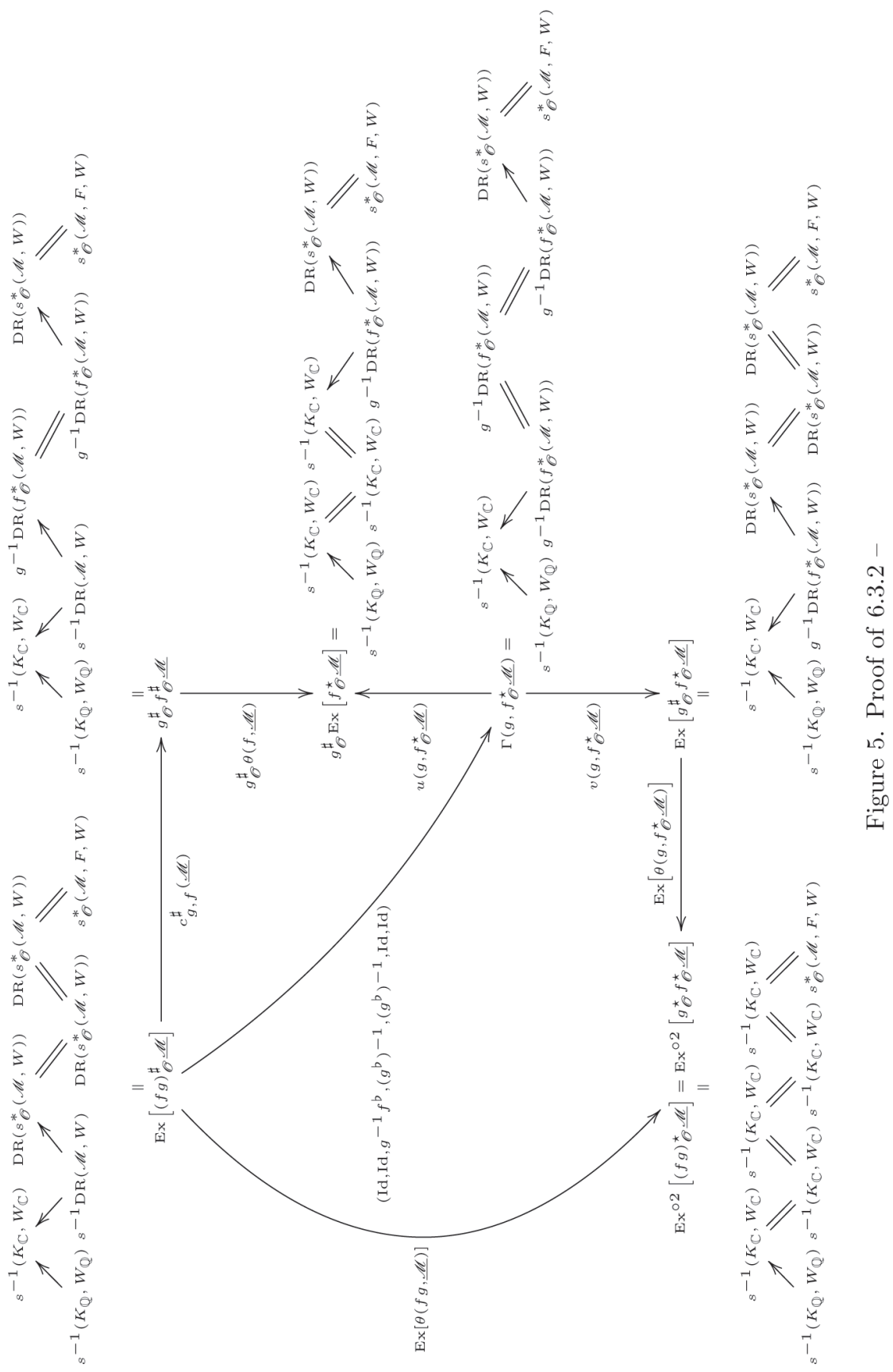




\section{REFERENCES}

[1] A. A. Beľlinson, Notes on absolute Hodge cohomology, Applications of algebraic $K$-theory to algebraic geometry and number theory, Part I, II (Boulder, Colo., 1983), Contemp. Math., vol. 55, Amer. Math. Soc., Providence, RI, 1986, pp. 35-68. MR MR862628 (87m:14019)

[2] A. A. Beİlinson, Correction to: "Notes on absolute Hodge cohomology" [applications of algebraic K-theory to algebraic geometry and number theory, part i, ii (Boulder, Colo., 1983), 35-68, Amer. Math. Soc., Providence, R.I., 1986; MR0862628 (87m:14019)], K-theory, arithmetic and geometry (Moscow, 1984-1986), Lecture Notes in Math., vol. 1289, Springer, Berlin, 1987, pp. 2526. MR MR923132 (89a:14023)

[3] A. A. BeǏlinson, On the derived category of perverse sheaves, $K$-theory, arithmetic and geometry (Moscow, 1984-1986), Lecture Notes in Math., vol. 1289, Springer, Berlin, 1987, pp. 27-41. MR MR923133 (89b:14027)

[4] A. A. BeÍlinson, J. Bernstein, and P. Deligne, Faisceaux pervers, Analysis and topology on singular spaces, I (Luminy, 1981), Astérisque, vol. 100, Soc. Math. France, Paris, 1982, pp. 5-171. MRMR751966 (86g:32015)

[5] Pierre Deligne, Théorie de Hodge. III, Inst. Hautes Études Sci. Publ. Math. (1974), no. 44, 5-77. MR 0498552 (58 \#16653b)

[6] Ryoshi Hotta, Kiyoshi Takeuchi, and Toshiyuki Tanisaki, D-modules, perverse sheaves, and representation theory, Progress in Mathematics, vol. 236, Birkhäuser Boston Inc., Boston, MA, 2008, Translated from the 1995 Japanese edition by Takeuchi. MR 2357361 (2008k:32022)

[7] Annette Huber, Realization of Voevodsky's motives, J. Algebraic Geom. 9 (2000), no. 4, 755-799. MR MR1775312 (2002d:14029)

[8] Annette Huber, Corrigendum to: "Realization of Voevodsky's motives" [J. Algebraic Geom. 9 (2000), no. 4, 755-799], J. Algebraic Geom. 13 (2004), no. 1, 195-207. MR MR2008720 (2004h:14030)

[9] Florian Ivorra, Mixed Hodge complexes on algebraic varieties and $t$ structure, Journal of Algebra 433 (2015) 107-167.

[10] Masaki Kashiwara, A study of variation of mixed Hodge structure, Publ. Res. Inst. Math. Sci. 22 (1986), no. 5, 991-1024. MR MR866665 (89i:32050)

[11] Masaki Kashiwara, Algebraic study of systems of partial differential equations, Mém. Soc. Math. France (N.S.) (1995), no. 63, xiv+72. MR 1384226 (97f:32012)

[12] Masaki Kashiwara and Pierre Schapira, Sheaves on manifolds, Grundlehren der Mathematischen Wissenschaften [Fundamental Principles of Mathematical Sciences], vol. 292, Springer-Verlag, Berlin, 1994, With a chapter in French by Christian Houzel, Corrected reprint of the 1990 original. MR 1299726 (95g:58222)

[13] G. Laumon, Sur la catégorie dérivée des D-modules filtrés, Algebraic geometry (Tokyo/Kyoto, 1982), Lecture Notes in Math., vol. 1016, Springer, Berlin, 1983, pp. 151-237. MR MR726427 (85d:32022)

[14] Marc Levine, Mixed motives, Mathematical Surveys and Monographs, vol. 57, American Mathematical Society, Providence, RI, 1998. MR 99i:14025

[15] Philippe Maisonobe and Zoghman Mebkhout, Le théorème de comparaison pour les cycles évanescents, Éléments de la théorie des systèmes différentiels 
géométriques, Sémin. Congr., vol. 8, Soc. Math. France, Paris, 2004, pp. 311389. MR 2077650 (2005k:32032)

[16] Zoghman Mebkhout, Le théorème de positivité, le théorème de comparaison et le théorème d'existence de Riemann, Éléments de la théorie des systèmes différentiels géométriques, Sémin. Congr., vol. 8, Soc. Math. France, Paris, 2004, pp. 165-310. MR 2077649 (2005h:32020)

[17] Morihiko Saito, Modules de Hodge polarisables, Publ. Res. Inst. Math. Sci. 24 (1988), no. 6, 849-995 (1989). MR MR1000123 (90k:32038)

[18] Morihiko Saito, Extension of mixed Hodge modules, Compositio Math. 74 (1990), no. 2, 209-234. MR MR1047741 (91f:32046)

[19] Moriniko SAito, Mixed Hodge modules, Publ. Res. Inst. Math. Sci. 26 (1990), no. 2, 221-333. MR MR1047415 (91m:14014)

[20] Morihiko Saito, Mixed Hodge complexes on algebraic varieties, Math. Ann. 316 (2000), no. 2, 283-331. MR MR1741272 (2002h:14012)

[21] Joseph Steenbrink and Steven Zucker, Variation of mixed Hodge structure. I, Invent. Math. 80 (1985), no. 3, 489-542. MR MR791673 (87h:32050a)

Manoscritto pervenuto in redazione il 23 giugno 2013. 
